\title{
The Dynamics of Coupled Oscillators
}

\author{
by \\ Nigel Lawrence Holland
}

A thesis

submitted to the Victoria University of Wellington

in fulfilment of the

requirements for the degree of

Master of Science

in Mathematics.

Victoria University of Wellington

2008 


\begin{abstract}
The subject is introduced by considering the treatment of oscillators in Mathematics from the simple Poincaré oscillator, a single variable dynamical process defined on a circle, to the oscillatory dynamics of systems of differential equations. Some models of real oscillator systems are considered. Noise processes are included in the dynamics of the system. Coupling between oscillators is investigated both in terms of analytical systems and as coupled oscillator models.

It is seen that driven oscillators can be used as a model of 2 coupled oscillators in 2 and 3 dimensions due to the dependence of the dynamics on the phase difference of the oscillators. This means that the dynamics are easily able to be modelled by a 1D or 2D map. The analysis of $\mathrm{N}$ coupled oscillator systems is also described.

The human cardiovascular system is studied as an example of a coupled oscillator system. The heart oscillator system is described by a system of delay differential equations and the dynamics characterised. The mechanics of the coupling with the respiration is described.

In particular the model of the heart oscillator includes the baroreceptor reflex with time delay whereby the aortic fluid pressure influences the heart rate and the peripheral resistance. Respiration is modelled as forcing the heart oscillator system.

Locking zones caused by respiratory sinus arrhythmia (RSA), the synchronisation of the heart with respiration, are found by plotting the rotation number against respiration frequency. These are seen to be relatively narrow for typical physiological parameters and only occur for low ratios of heart rate to respiration frequency. Plots of the diastolic pressure and
\end{abstract}


heart interval in terms of respiration phase parameterised by respiration frequency illustrate the dynamics of synchronisation in the human cardiovascular system. 


\section{Acknowledgments}

I acknowledge my parents, the patience of my supervisor Mark McGuinness, and the Victoria University library. 


\section{Contents}

1 Introduction $\quad 1$

2 Oscillators in Mathematics 3

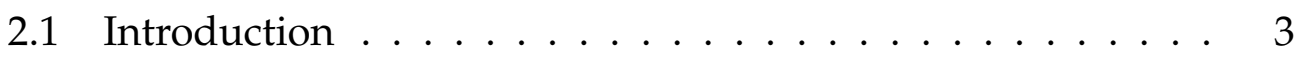

2.2 Relaxation Oscillators . . . . . . . . . . . . . . . 3

2.3 Poincaré Oscillator . . . . . . . . . . . . . . . . . 4

2.4 Classical Oscillators . . . . . . . . . . . . . . 7

2.5 Oscillating Systems . . . . . . . . . . . . . . . . . . . . . . . . . . . . . . .

2.6 Measurement of Oscillator Phase . . . . . . . . . . . . . . . 12

2.7 Noise in Oscillators . . . . . . . . . . . . . . . . . . . 15

3 Coupled Oscillators $\quad 23$

3.1 Introduction . . . . . . . . . . . . . . 23

3.2 Nonisochronous driven oscillator . . . . . . . . . . . . . . . . 24

3.2.1 Introduction . . . . . . . . . . . . . 24

3.2.2 Weak Coupling . . . . . . . . . . . . . 27

3.2.3 Quasiperiodic behaviour . . . . . . . . . . 27

3.2.4 Structure of Arnolds tongues . . . . . . . . . . . . 28

3.2 .5 Strong coupling . . . . . . . . . . . . . 30

3.3 Dynamics of phase locking . . . . . . . . . . . . . 34

3.3.1 Deterministic isochronous oscillators . . . . . . . 34

3.3.2 Example of forced oscillator . . . . . . . . . . 37

3.3.3 Chaotic oscillators .............. 41 
CONTENTS iii

3.4 Perturbed Nonisochronous Oscillator . . . . . . . . . . . 43

3.4 .1 Introduction . . . . . . . . . . . . . . 43

3.4 .2 Planar dynamics . . . . . . . . . . . . . 43

3.4.3 Hopf bifurcation . . . . . . . . . . . . . . 44

3.4.4 Global bifurcations . . . . . . . . . . . . . 45

3.4.5 Dynamics near a Saddle point . . . . . . . . . . . 46

3.4.6 Bifurcations near a Saddle point . . . . . . . . . . 49

3.5 Method of Averaging . . . . . . . . . . . . . . 52

3.5 .1 Introduction . . . . . . . . . . . . . . . . 52

3.5.2 Co-moving coordinates . . . . . . . . . 53

3.5.3 Averaging Theorem .............. 53

3.5.4 Validity of Averaging method . . . . . . . . . . . . 54

3.6 Action/Angle coordinates . . . . . . . . . . . . 56

3.6.1 Hamiltonian oscillators . . . . . . . . . . . . 56

3.6.2 System action variables . . . . . . . . . . . 57

3.6.3 Generating function . . . . . . . . . . . . 57

3.6.4 Perturbed integrable systems . . . . . . . . . . 58

3.6.5 Dynamics near resonant trajectories . . . . . . . . . 59

3.7 Melnikov's method . . . . . . . . . . . . . . . . . . 60

3.7 .1 Introduction . . . . . . . . . . . . . . . 60

3.7.2 Geometry of a homoclinic loop . . . . . . . . . 60

3.7.3 Bifurcations of a homoclinic loop . . . . . . . . . 62

4 Mathematical Modelling of the Cardiovascular System 64

4.1 Introduction . . . . . . . . . . . . . . . . . . . . . . 64

4.2 Background . . . . . . . . . . . . . . 65

4.3 Studying Synchronisation in the Cardiovascular System . . . . . . . . . . . . . . . . . . . 69

4.3.1 Numerical solution of system . . . . . . . . . . 73

4.3.2 Behaviour of heart system model . . . . . . . . . 76

4.3.3 Frequency response of heart system . . . . . . . . 83 
CONTENTS iv

5 Coupling in the Cardiovascular System $\quad 90$

5.1 Introduction . . . . . . . . . . . . . . . . . 90

5.2 A Phase locked loop model of

Cardiovascular system synchronisation . . . . . . . . . 92

5.3 The Synchronization mechanism in

the Cardiovascular system . . . . . . . . . . . . . . . . . 100

5.4 RSA Synchronization detection . . . . . . . . . . . . . 106

5.5 RSA locking regions of the Cardiovascular system . . . . . . 116

5.6 Dynamics of RSA phase locking . . . . . . . . . . . . . . 123

5.6.1 Step response of heart system . . . . . . . . . . . . 123

5.6.2 Phase relationship across locking region . . . . . . . . 124

5.6.3 Maps of the cardiovascular system . . . . . . . . . . 124

6 Conclusions

135

A Physiology of the Cardiovascular System 146 


\section{Chapter 1}

\section{Introduction}

Coupling between oscillators was first studied by Huygens in 1673 who noticed that pendulum clocks in the same room became synchronised. In 1889 Henri Poincare laid the foundations of dynamical systems theory in his study of the stability of the solar system. His work was an entry in a contest to commemorate the 60th birthday of King Oscar II of Sweden. Poincare uncovered the significance of the homoclinic points at the crossing of the unstable and stable manifolds and also introduced the Poincare map. Coupled and synchronised oscillators are significant in electronics also. The ubiquitous phase-locked loop is an example. The dynamics of a phase-locked loop can be highly nonlinear and are not fully understood. For example chaotic dynamics and locking region hysteresis are possible. Commonly only a simplified linear model of the dynamics is used.

Coupled oscillators also occur in biological systems. One early use of mathematics to model biological systems was Van der Pol's use in 1928 of a driven Van der Pol oscillator to explain some normal, and pathological, rhythms of the heart. However Van der Pol devised his differential equation to model an electronic oscillator based on the triode valve. In the human cardiovascular system coupling between respiration and the heart results in synchronisation at a fixed $\frac{m}{n}$ ratio for some respiration frequencies. This results in the heart rate being entrained by the respi- 
ration so over a zone of respiration frequencies the heart rate maintains the fixed ratio. This process of entrainment is common to all systems of coupled oscillators. The zones, which correspond to the rational numbers, are known as Arnold Tongues after V.I. Arnold who discovered them in 1963. While the dynamics are quite simple at low coupling, consisting of zones of synchronisation the boundaries of which are a tangent bifurcation, interspersed with quasiperiodic regions where the frequencies are incommensurate, at higher coupling chaotic behaviour is possible with a more elaborate bifurcation structure.

Arnold Tongues are generic to coupled oscillators and there is a range of literature on their theory and the results of mathematical modelling. There is less literature on their occurence in biological systems and so what follows explains the results of modelling the human cardiovascular system using Matlab and the DDE23 differential delay equation solver. The locking zones found in the model should be comparable to physiological data. The dynamics of cardiovascular synchronisation and the mechanics of synchronisation in the human cardiovascular system are explored. 


\section{Chapter 2}

\section{Oscillators in Mathematics}

\subsection{Introduction}

A fundamental characteristic of oscillators is a repeating sequence of states. The progression of states is measured by the phase of the oscillator. For a map $x_{i+1}=f\left(x_{i}\right)$ an order $n$ fixed point exists when $x_{i+n}=x_{i}$ where $f$ is a $\mathcal{C}^{1}$ homeomorphism. The iteration $i$ indicates the progression of the phase wrt the initial point. As a dynamical system defined by a vector field on a differential manifold, an oscillator is a piecewise continuous diffeomorphism $f(\theta)$ on $T^{1}$ or $S^{1}$ which has the range $[0 \ldots 1)$ or $[0 \ldots 2 \pi)$. What are the possible dynamics of a single dimensional dynamical system? The dynamics could tend to an equilibrium point or they must tend in one direction for all time. So for an oscillator the vector field on $\mathbb{R}^{1}$ must be piecewise continuous, periodic in $\theta$, and there must be a constant term so that an equilibrium point is not possible.

\subsection{Relaxation Oscillators}

The integrate-and-fire relaxation oscillator explicitly models episodic behaviour and is commonly used in modelling biological systems (A.A. Brailove 
[1]). Most generally the integrate-and-fire oscillator is described by a quantity $x(t)$ which increases dynamically according to a charging process. At a fixed upper threshold the process changes to a discharge to a lower threshold level where the cycle repeats itself. It is a useful simplification to assume that the discharge process occurs instantaneously. C.S. Peskin [2] described the dynamics of the charging process by $\frac{\mathrm{d} x}{\mathrm{~d} t}=S_{0}-\gamma x$ which can also model the charging of a capacitor through a resistor by a constant voltage source $\frac{S_{0}}{\gamma}$. Mirollo and Strogatz [3] formalised the idea of the state variable $x(t)$. The state of the oscillator is completely determined by the single variable $x$ which increases monotonically in time, from a lower threshold of zero to an upper threshold of one, at which point the oscillator fires and $x$ is instantly reset to zero. The phase of the oscillator $\phi$, is related to the state variable by $x=f(\phi)$. The function $f(\phi)$ is defined such that $\frac{\mathrm{d} \phi}{\mathrm{d} t}=$ const $=\nu$, the free running frequency of the oscillator in cycles per second. The phase is defined to be zero when the state $x$ is zero. A further assumption is that $f(\phi)$ is concave downwards as it is for the voltage vs time curve of the example of the resistor and charging capacitor. For the example of the resistor and capacitor $x(t)=\frac{S_{0}}{\gamma}\left(1-\mathrm{e}^{-\gamma t}\right)$. The function $f(\phi)=x$ is found by substituting $\phi=\nu t$ so that $f(\phi)=\frac{1-\mathrm{e}^{-\frac{\phi \gamma}{\nu}}}{1-\mathrm{e}^{-\frac{\gamma}{\nu}}}$, where the denominator is found from the upper threshold of $x(t)=1$ at $t=\frac{1}{\nu}$.

\subsection{Poincaré Oscillator}

Wanzhen, Glass, and Shrier [4] describe a 2D system of differential equations which is called the Poincaré oscillator as probably the simplest stable limit cycle oscillator, which has been considered many times as a model of biological oscillations. Radial coordinates are natural, so

$$
\begin{aligned}
& \frac{\mathrm{d} \phi}{\mathrm{dt}}=\frac{1}{T_{0}}, \\
& \frac{\mathrm{d} r}{\mathrm{~d} t}=k r(1-r),
\end{aligned}
$$


where the two variables $r$ and $\phi$ represent the distance from the origin, and the angle or phase, respectively. Any value of $r$ except 0 evolves to $r=1$. Evidently $r=0$ is an unstable equilibrium point. $\phi$ increases at a constant rate and is evaluated modulo $1 . \phi$ is not defined at the origin and is a phase singularity there.

What is the effect of an external perturbation on the trajectory? $r$ decays back to $r=1$, depending on $k$, and $\phi$ is displaced to a new phase $\phi^{\prime}$. Wanzhen et al. consider as a perturbation a horizontal translation of magnitude $b$. This causes a shift of variables $\left(r_{0}, \phi_{0}\right)$ to $\left(r^{\prime}, \phi^{\prime}\right)$ where

$$
\begin{aligned}
& \phi^{\prime}=\frac{1}{2 \pi} \cos ^{-1}\left(\frac{b+r_{0} \cos \left(2 \pi \phi_{0}\right)}{r^{\prime}}\right), \\
& r^{\prime}=\sqrt{r_{0}^{2}+b^{2}+2 r_{0} b \cos \left(2 \pi \phi_{0}\right)} .
\end{aligned}
$$

Two cases can be distingushed depending on the winding number of the resetting. The winding number is the relative change in phase of $\phi^{\prime}$ while $\phi_{0}$ changes in phase from 0 to 1 . Thus for type 1 or 'weak' resetting $\phi^{\prime}$ goes through the equivalent change of phase as $\phi_{0}$, and for type 0 or 'strong' resetting, the change in phase of $\phi^{\prime}$ is zero. The type of the resetting is synonomous with the winding number. Geometrically the two cases can be distinguished by whether or not the original $r=1$ trajectory is displaced past the phase singularity at the origin. Wanzhen et al. further consider the effect of multiple pulse resetting and naturally find a dependance on $k, b$, and $\delta$, the time between the reset pulses.

The model is compared with experimental results of stimulation of chicken heart cell aggregates and good agreement is found but it is noted that this does not mean that the Poincare oscillator represents an accurate model of the cardiac preparation but that it captures the essential topological properties of biological oscillators such as the heart cell aggregate.

Further understanding of the effect of phase resetting on an oscillator can be found through the notion of isochrons. An isochron is a line or surface in the phase space of the system from which the trajectories evolve to equal phase. In the case of the Poincaré oscillator system it can be seen 
that depending on $k$ the trajectories of points in the phase plane lying on an isochron will converge to the same phase on the limit cycle $r=1$ as time progresses. If the change in phase $\frac{\mathrm{d} \phi}{\mathrm{d} t}$ does not depend on $r$ then the isochron is aligned with the component of the phase line in $r$. The curvature of the isochron reflects the change in magnitude of the $\theta$ component with $r$. In the case of the Poincare oscillator it can be the seen that the isochrons are radial lines.

Clearly the isochrons converge at the phase singularity at the origin where the phase is undefined. Evidently the phase of points near the origin can exhibit large changes in response to relatively small perturbations. A.T. Winfree [5] terms the phaseless manifold of the singularity where the phase is undefined a 'black hole'. In biological systems this can be a region of phase space rather than a point. Winfree gives an example of the construction of isochrons for limit cycle attractor systems with polar symmetry, that is $\frac{\mathrm{d} \phi}{\mathrm{d} t}$ depending only on $r$, which generalize the Poincaré oscillator of Wanzhen et al. Let $\frac{\mathrm{d} \phi}{\mathrm{d} t}=A(r)$ with the unit of time chosen so that at the radius of the limit cycle attractor $A\left(r_{0}\right)=1$, and $\frac{\mathrm{d} r}{\mathrm{~d} t}=B(r)$ with $B\left(r_{0}\right)=0$ and $\frac{\mathrm{d} B}{\mathrm{~d} r}<0$ at $r_{0}$. The isochrons must have polar symmetry so $\varphi=g(\phi, r)=\phi-f(r)$, that is the difference in phase of the isochron at state $(\phi, r)$ from $\phi$ depends only on $r$. The rate of change of the isochron $\varphi$ at the state $(\phi, r)$ must then be the same as $\phi$ on the limit cycle, that is 1 , so

$$
\frac{\mathrm{d} \varphi}{\mathrm{d} t} \equiv 1=\frac{\mathrm{d} \phi}{\mathrm{d} t}-\frac{\mathrm{d} f(r)}{\mathrm{d} r} \frac{\mathrm{d} r}{\mathrm{~d} t},
$$

and

$$
\frac{\mathrm{d} f}{\mathrm{~d} r}=\frac{\frac{\mathrm{d} \phi}{\mathrm{d} t}-1}{\frac{\mathrm{d} r}{\mathrm{~d} t}} .
$$

$f(r)$ is obtained by integrating this equation and thus $g(\phi, r)$.

For example, consider the system:

$$
\begin{aligned}
& \frac{\mathrm{d} r}{\mathrm{~d} t}=5(1-r)\left(r-\frac{1}{2}\right) r, \\
& \frac{\mathrm{d} \phi}{\mathrm{d} t}=1+\varepsilon(1-r) .
\end{aligned}
$$


Solution is via partial fractions, the identities, $\frac{\mathrm{d} \ln (x)}{\mathrm{d} x}=\frac{1}{x}, \frac{\mathrm{d} \ln \left(\frac{1}{x}\right)}{\mathrm{d} x}=\frac{-1}{x},|x|>$ 0 , and simplifying radicals, so

$$
\begin{aligned}
r(t) & =\frac{-2+\frac{1}{2} C \mathrm{e}^{\frac{5}{2} t} \pm \frac{1}{2}\left(C^{2} \mathrm{e}^{5 t}-4 C \mathrm{e}^{\frac{5}{2} t}\right)^{\frac{1}{2}}}{C \mathrm{e}^{\frac{5}{2} t}-4} \\
\phi(t) & =\int \varepsilon r(t) \mathrm{d} t+t+\varepsilon t+D \\
\varphi & =\phi-\frac{2 \varepsilon}{5} \ln \left(2-\frac{1}{r}\right)
\end{aligned}
$$

Note that $r=1$ is a stable attractor, $r=\frac{1}{2}$, is an unstable periodic solution, and $r=0$, is a fixed point, so the region $r<\frac{1}{2}$ is attracted to $r=0$, and the region $r>\frac{1}{2}$ is attracted to $r=1$. See fig 2.1. A phase space consisting of a periodic attractor and an attracting fixed point occurs often in biological systems. An external signal shifts the system between the two states.

\subsection{Classical Oscillators}

One classical oscillator is the damped pendulum:

$$
\frac{\mathrm{d}^{2} \theta}{\mathrm{d} t^{2}}+\delta \frac{\mathrm{d} \theta}{\mathrm{d} t}+\sin (\theta)=0 .
$$

Naturally the solution of (2.12) is periodic in $\theta$ consisting of fixed points on the $\theta$ axis at $\theta=2 n \pi$. The phase lines spiral into a fixed point depending on the value of the damping $\delta$. If $\delta=0$ the phase lines orbit a fixed point or monotonically increase in $\theta$, in a narrow range of $\frac{\mathrm{d} \theta}{\mathrm{d} t}$. In this case the system is conservative and the phase lines are the solution of the equation $\nabla \phi=0$, where $\phi$ is a scalar function.

The well known double well Duffing equation:

$$
\frac{\mathrm{d}^{2} x}{\mathrm{~d} t^{2}}+\delta \frac{\mathrm{d} x}{\mathrm{~d} t}+x^{3}-x=0
$$

is a specialization of a larger class of equations given by (2.14), usually referred as Duffing equations:

$$
\frac{\mathrm{d}^{2} x}{\mathrm{~d} t^{2}}+\delta \frac{\mathrm{d} x}{\mathrm{~d} t}+\frac{\mathrm{d} P(x)}{\mathrm{d} x}=0
$$




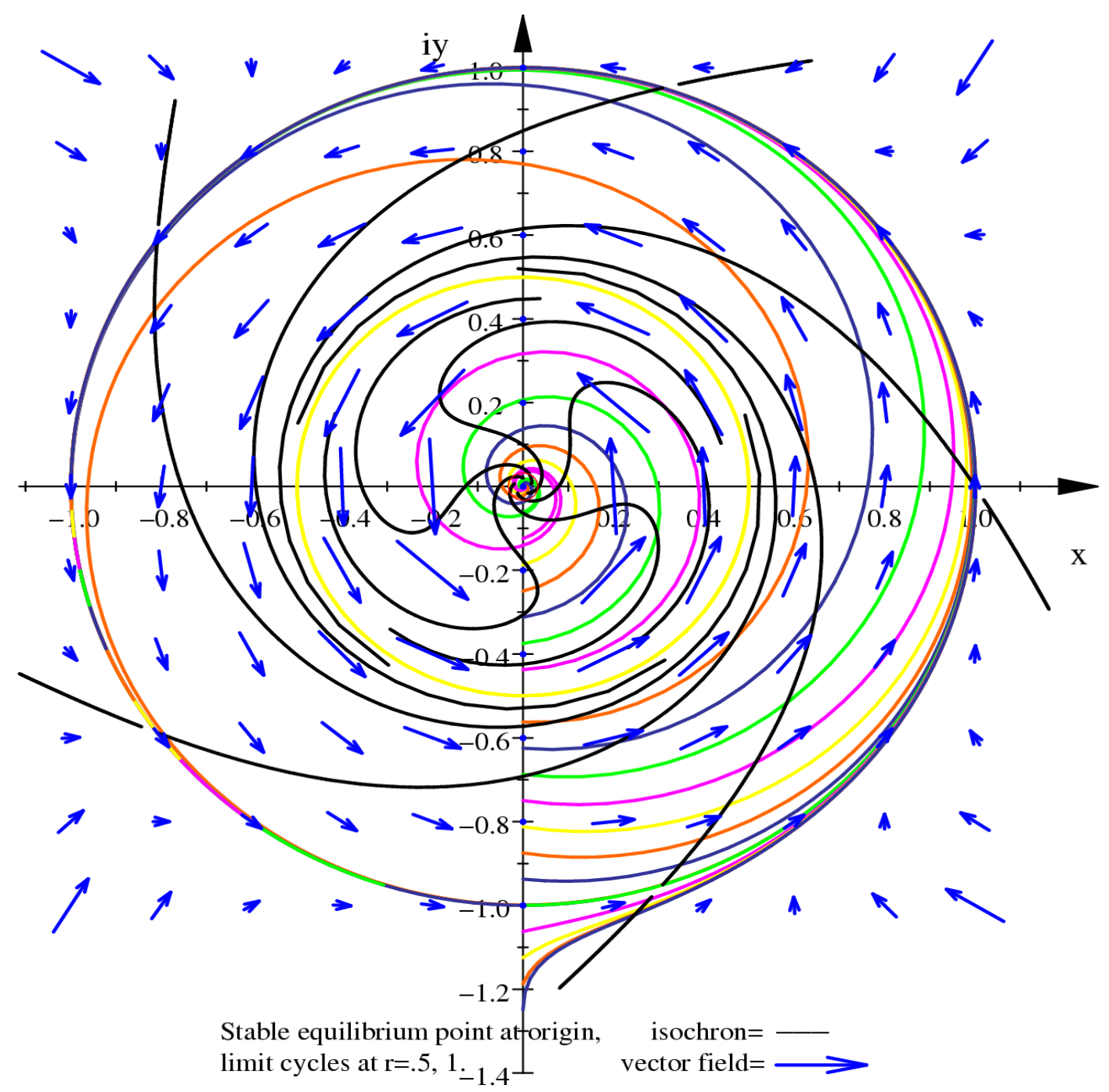

Figure 2.1: nonisochronous oscillator with oscillator annihilation in phase plane 
where $P(x)$ is the equation for a potential energy field. In Physics, work equals force times distance, which explains the use of $\frac{\mathrm{d} P(x)}{\mathrm{d} x}$. It can be seen in the case of (2.12) that the potential function is $-\cos (\theta)$, and the minimums of potential energy are at $2 n \pi$. In the case of (2.13) $P(x)=$ $x^{4}-\frac{1}{2} x^{2}+\delta$, so the $-x^{2}$ term causes a peak in the middle of the $x^{4}$ term leaving two wells. If the potential function $-\cos (\theta)$ is approximated by its first 3 terms, $-1+\frac{1}{2} \theta^{2}-\frac{1}{24} \theta^{4}$ then this is the negative of the potential function of the double well Duffing equation and a single potential well exists on the peak of the $-\theta^{4}$ term.

B. van der Pol [6] devised the Van der Pol oscillator to model an electronic oscillator, using a parallel inductor and capacitor as the resonator, inductively coupled to a triode valve as the amplifier. In contrast to the Duffing oscillator which has a proportionate damping term $\delta$, the damping in the Van der Pol oscillator is dependent on the amplitude, so that a limit cycle attractor exists in the phase plane. This is the effect of introducing a nonlinearity in the transfer curve of the amplifier so that $i=\alpha v-\gamma v^{3}$, where $i$ is current and $v$ is voltage, compared to the linear model of the amplifier $i=\alpha v$ for a linear oscillator. The Van der Pol equation is:

$$
\frac{\mathrm{d}^{2} v}{\mathrm{~d} t^{2}}-\alpha\left(1-v^{2}\right) \frac{\mathrm{d} v}{\mathrm{~d} t}+\omega^{2} v=0 .
$$

If the parameter $\epsilon=\frac{\alpha}{\omega} \ll 1$, then the solution is a free sinusoidal-like oscillation, if $\epsilon \gg 1$ (heavily damped) then the solution is like a relaxation oscillation. The relaxation oscillator solution is significant. Compared to the state variable of the ordinary relaxation oscillator it is continuous whereas the ordinary oscillator has a simple unparametrisable discontinuity at the threshold.

Van der Pol observed that for small values of $v$ the damping term is -ve so that the amplitude of the oscillation increases and the sign of the damping changes to limit the amplitude of the oscillation. This suggested to try as a solution a cosinusoid with a slowly varying amplitude,

$$
v=a(\tau) \cos (\tau),
$$


with $\tau=\omega t$, where slowly is taken as meaning that during one cycle the percentage amplitude change is small, that is $\frac{1}{a} \mathrm{~d} a \ll 1$. As the nonlinearity is assumed small the solution will nearly be sinusoidal so higher harmonics can be neglected. Also neglecting higher derivatives of $a(\tau)$, and using $\frac{\mathrm{d} a}{\mathrm{~d} \tau} \ll a$ to neglect terms with coefficients in $\frac{\mathrm{d} a}{\mathrm{~d} \tau}$, substituting (2.16) into (2.15) gives,

$$
\frac{\mathrm{d} a^{2}}{\mathrm{~d} \tau}-\epsilon\left(a^{2}-\frac{1}{4} a^{4}\right)=0,
$$

which results in,

$$
v=\frac{2 \cos (\tau)}{\sqrt{1+\mathrm{e}^{-\epsilon\left(\tau-\tau_{0}\right)}}} .
$$

The observation that at small $v$ the damping term is -ve and so the oscillation amplitude must increase shows that a small amount of noise will cause the oscillator to converge to a limit cycle of amplitude equal to 2 .

An extension of the Van der Pol oscillator is the Bonhöffer-van der Pol equation. This is a simplified model of the Hodgkin-Huxley system for the firing of a squid neuron, devised by FitzHugh and Nagumo [7], [8]. They reduced the Hodgkin-Huxley equation (four-dimensional) to a two dimensional system called the BVP equation or FitzHugh-Nagumo model by extracting the excitability of the dynamics in the Hodgkin-Huxley equation. The system is:

$$
\begin{gathered}
C \frac{\mathrm{d} v}{\mathrm{~d} t}=-i-g(v), \\
L \frac{\mathrm{d} i}{\mathrm{~d} t}=v-r i+E .
\end{gathered}
$$

This can be seen as an extension of the Van der Pol oscillator by substitution, giving:

$$
\frac{\mathrm{d}^{2} v}{\mathrm{~d} t^{2}}+\left(\frac{r}{L}+\frac{1}{C} \cdot \frac{\mathrm{d} g(v)}{\mathrm{d} v}\right) \cdot \frac{\mathrm{d} v}{\mathrm{~d} t}+\frac{v+r \cdot g(v)+E}{L C}=0 .
$$

For a +ve cubic nonlinearity $g(v)$ the damping is comparable to the standard Van der Pol oscillator. The potential well is a -ve quartic as for the 
truncated version of the simple pendulum and can be seen to have a periodic attractor at a root of $\frac{v+r . g(v)+E}{\mathrm{LC}}$. The effect of the $E$ bias term is a linear term in the potential well which skews the minimum to one side of the well corresponding to the characteristic of the biological system where the trajectories of the periodic orbits are squeezed closer to the fixed point for part of the orbit.

\subsection{Oscillating Systems}

Oscillators, that is periodic attractors, also occur in higher dimensional systems. They are represented by a stable limit cycle in the phase space of an autonomous dissipative dynamical system.

Theorem 2.5.1. A bounded orbit of an autonomous flow, that is a trajectory that evolves entirely as a time independent function of its own state variables, either has a Lyapunov exponent equal to zero or else it has an equilibrium point in its $\omega$-limit set.

Proof. If the latter is not the case let $f$ be a piecewise continuous system, then $0<b<\left|f\left(F_{t}(v)\right)\right|<B, \forall t$, for some positive bounds $b$ and $B$, where $F_{t}(v)$ is the solution of the system $f$ with initial condition $v$ at time $t$. If $r(n)$ is the expansion in the direction of $f(v)$ after $n$ time units ( $n$ steps of the $t=1$ map), then

$$
0 \leqslant \lim _{n \rightarrow \infty} \frac{1}{n} \ln b \leqslant \lim _{n \rightarrow \infty} \frac{1}{n} \ln r(n) \leqslant \lim _{n \rightarrow \infty} \frac{1}{n} \ln B \leqslant 0 .
$$

Therefore the Lyapunov exponent in the direction tangent to the orbit is zero.

The phase corresponds to motion along a limit cycle, that is in the direction in which neither expansion or contraction of the phase volume occurs, therefore the phase of oscillations and this direction in phase space, corresponds to the zero Lyapunov exponent. It is natural for phase to be 
proportional to time, and increasing by $2 \pi$ for each period $T_{0}$ of the limit cycle, so the dynamics of the phase on the limit cycle can be described as,

$$
\frac{\mathrm{d} \phi}{\mathrm{d} t}=\omega_{0},
$$

where $\omega_{0}=\frac{2 \pi}{T_{0}}$. The correspondence of phase to the zero Lyanpunov exponent shows why it is amenable to small external actions. Whereas perturbations in amplitude relax to the stable limit cycle value, perturbations in phase accumulate.

\subsection{Measurement of Oscillator Phase}

Oscillators can also be defined in chaotic systems, that is systems with a Lyapunov exponent greater than zero. In this case as the trajectory is bounded there must be a Lyapunov exponent equal to zero also. The state of the oscillation phenomenon is measured by the phase of the oscillator. The phase variable is defined in the direction of the zero Lyapunov exponent corresponding to shifts along the flow, as a piecewise linear function of time so that an increment of $2 \pi$ occurs at each return to a Poincaré secant surface:

$$
\phi_{P}(t)=2 \pi \frac{t-t_{n}}{t_{n+1}-t_{n}}+2 \pi n, t_{n} \leqslant t<t_{n+1},
$$

where $t_{n}$ is the time of the $n$-th crossing of the secant surface. Periodic oscillations would correspond to a fixed point of the Poincaré map. Pikovsky et al.[9] give two other methods to define the phase.

I. It often happens that the strange attractor can be projected onto a plane and the projection revolves around a centre point which can be taken as an origin from which the angle between a vector to the point of the trajectory, and a line in the plane taken to be the reference zero phase, is the instantaneous phase $\phi(t)$.

II. Taking any oscillatory observable $s(t)$ of a chaotic system the so- 
called analytic signal $\zeta(t)$ ( D. Gabor[10]) can be constructed:

$$
\zeta(t)=s(t)+i s_{H}(t)=A(t) \mathrm{e}^{i \phi(t)},
$$

where $s_{H}(t)$ is the Hilbert transform (HT) of $s(t)$,

$$
s_{H}(t)=\pi^{-1} P . V . \int_{-\infty}^{\infty} \frac{s(\tau)}{\tau-t} d \tau,
$$

(where P.V. means the Cauchy principal value). The instantaneous amplitude $A(t)$, and the instantaneous phase $\phi(t)$ are then unambiguously defined from (2.23). From (2.24) the Hilbert transform $s_{H}(t)$ of $s(t)$ may be considered to be the convolution of the functions $s(t)$ and $\frac{1}{\pi t}$. Hence the Fourier transform $S_{H}(j \omega)$ of $s_{H}(t)$ is the product of the Fourier transform $S(j \omega)$ of $s(t)$ and of $\frac{1}{\pi t},(-j$, as an odd function gives a purely imaginary transform). For physically relevant frequencies $\omega>0, S_{H}(j \omega)=-j S(j \omega)$; i.e. $S_{H}(j \omega)$ is $S(j \omega)$ shifted in phase by a constant $\frac{\pi}{2}$ phase lag.

For example consider a Rössler system:

$$
\begin{aligned}
\dot{x} & =-\omega y-z, \\
\dot{y} & =\omega x+a y, \\
\dot{z} & =.4+z(x-8.5) .
\end{aligned}
$$

The motion of the chaotic attractor of this system is such that orbits continually circulate around the $z$ axis. Elimination of $x$ from (2.25) shows that the system is oscillatory with frequency $\omega$.

$$
\ddot{y}-a \dot{y}+\omega^{2} y=-\omega z
$$

Changing $x$ and $y$ into polar coordinates $r=\left(x^{2}+y^{2}\right)^{\frac{1}{2}}, \phi_{P}=\arcsin \left(\frac{y}{r}\right)$ and taking the phase as $\phi_{P}$ corresponds to a projection of the phase space onto the $x-y$ plane with $(0,0)$ as the origin of the attractor. This gives an average velocity of rotation i.e. the average return time to a Poincare surface, corresponding to the main peak in the power spectrum (Rosenblum et al.,[11]). 
The three methods give practically coinciding results for these examples (A.S. Pikovsky et al.,[9]). The growth of phase of a chaotic system cannot generally be expected to be uniform. It can be seen that the Poincaré return times (the instantaneous period) depend on the intersection of a chaotic amplitude with the Poincaré surface. Considering the Poincaré map the dynamics can be represented as (A.S. Pikovsky et al.,[9]):

$$
\begin{array}{r}
A_{n+1}=T\left(A_{n}\right), \\
\frac{\mathrm{d} \phi}{\mathrm{d} t}=\omega\left(A_{n}\right) \equiv \omega_{0}+F\left(A_{n}\right) .
\end{array}
$$

The amplitude $A$ is given by the coordinates on the Poincare section surface, and the transformation $T$ defines the Poincaré map, as $A_{n+1}$ is a function of $A_{n}$. The return time also depends on $A_{n}$ so the instantaneous frequency $\omega=\frac{2 \pi}{\left(t_{n+1}-t_{n}\right)}$ can be stated in terms of the average frequency $\omega_{0}$ and a term representing the effect of the chaotic amplitude $F\left(A_{n}\right)$. This can be treated stochastically, but is deterministic. While $T$ might be difficult to determine for a particular system it can be studied analytically by using a prototype chaotic map such as the logistic map or the tent map. $F$ might be approximated by Gaussian noise but a chaotic signal will not necessarily have these statistics. As the $A_{n}$ express the chaotic nature of the phase $F$ can also be taken to be $\delta A_{n}$, where $\delta$ is a small proportionality constant.

The dynamics of the phase are generally diffusive so for large $t$ it is expected that

$$
\left\langle\left(\phi(t)-\phi(0)-\omega_{0} t\right)^{2}\right\rangle \sim D_{P} t
$$

where the diffusion constant $D_{P}$ determines the phase coherence of the chaotic oscillations. This is the mean square of the deviation of the phase from the mean $\left(=\omega_{0} t\right)$ at time $t$ that is to say the variance at time $t .\langle\ldots\rangle=$ $\lim _{N \rightarrow \infty} \frac{1}{N} \Sigma_{i=0}^{N-1}\left(\phi_{i}(t)-\phi_{i}(0)-\omega_{0} t\right)^{2}$ is the ensemble average. Roughly speaking the diffusion constant is proportional to the width of the spectral peak of the chaotic attractor J.D. Farmer [12], i.e. $Q \simeq \frac{\sqrt{\omega_{1} \omega_{2}}}{\left(\omega_{2}-\omega_{1}\right)}$ where $\omega_{1}, \omega_{2}$ are the $-3 \mathrm{~dB}$ points of the power spectral peak, so $D_{p} \sim \frac{1}{Q}$. 
The Rössler attractor with $\omega=1$ and $a=.15$ gives $D_{P}<10^{-4}$ so the peak is very sharp and the attractor can be called phase coherent,[9].

\subsection{Noise in Oscillators}

The effect of noise can be analysed by adding a noise term $\xi(t)$ to the oscillator. Effectively a free-running oscillator is now driven by the noise term. The noise can be modelled as a Markov process, that is an ideal point process, a process without after effect. This has the advantage that Markov theory can be used to solve first passage time problems so this type of problem is the most significant for an oscillator as it represents the situation where an oscillator might jump a cycle.

For the theory of Markov processes to be applied the noise events must be independent. The noise events are said to be independent when they are uncorrelated. The correlation between noise events is given by the correlation time $\tau_{\text {corr }}$ of the noise signal. This is the time $\tau$ where the autocorrelation of the noise signal becomes insignificant compared with $\tau=0$. The autocorrelation of a signal is the quantity $C(\tau)=\langle\phi(t) \phi(t+$ $\tau)\rangle \equiv \lim _{N \rightarrow \infty} \frac{1}{N} \sum_{i=0}^{N} \phi_{i}(t) \phi_{i}(t+\tau)$, which is the ensemble average. If $\phi(t)$ is stationary, that is its statistical parameters are independent of $t$, then $C(\tau)=\lim _{T \rightarrow \infty} \frac{1}{T} \int_{0}^{T} \phi(t) \phi(t+\tau) d t$ the time average. In the current application the theory of Markov processes is applicable where $\tau_{\text {corr }} \ll \frac{1}{\varepsilon \omega_{0}}$. This implies that the correlation time $\tau_{\text {corr }}$ of the random process is much less than relaxation time of the dynamical process controlling the noise as $\varepsilon \omega_{0}$ is the first order linear term of the damping of a nonlinear oscillator or the damping coefficient of a linear oscillator. Effectively this means that the noise events are independent where the relaxation time of the noise process is much greater than the correlation time of the noise process. If the correlation time is much longer than the relaxation time then the noise dynamical process is ineffective as the transient response has decayed within the duration of the noise event. The lack of after effect 
justifies the use of Markov theory. The random process $\xi(t)$ is taken to be delta correlated, that is $\tau_{\text {corr }}=0$. It is described by correlation functions $k_{s}\left(t_{1}, \ldots t_{s}\right)=K_{s}(x) \delta\left(t_{1}-t_{2}\right) \ldots \delta\left(t_{1}-t_{s}\right)(s=2,3, \ldots) . K_{s}(x)$, where $x(t)=x$ at time $t$, are the $s$ order intensity coefficients,see R.L. Stratonovich [13]. A stationary Gaussian delta-correlated process with zero mean has $k_{2}\left(t_{1}, t_{2}\right)=K_{2} \delta\left(t_{1}-t_{2}\right)$ and only $K_{2}$ is nonzero. Taking the noise to be Gaussian $\delta$-correlated such that $\left\langle\xi(t) \xi\left(t^{\prime}\right)\right\rangle=2 D \delta\left(t-t^{\prime}\right),\langle\xi(t)\rangle=0$ allows a detailed description of the effect. $\xi(t)$ is a totally uncorrelated signal and the power spectrum is a constant $(=2 D)$ corresponding to white noise, as shown by the Wiener-Khinchin relation. Therefore for a 1D oscillator,

$$
\frac{\mathrm{d} \phi}{\mathrm{d} t}=\omega_{0}+\xi(t)
$$

The oscillator noise process can be viewed dynamically as the overdamped motion of a particle in a potential field, in this case $\frac{\partial P}{\partial x}=-\omega_{0}^{2} \phi$, reflecting that the effect of the noise is altered due to the potential field force as a function of the phase of the oscillator, and because $\tau_{\text {corr }} \ll \frac{1}{\varepsilon \omega_{0}}$, the acceleration being negligible as the particle is assumed massless, the particle motion is forced by the external force $\xi(t)$. The correspondence is that the impulsive frequency noise causes jumps in phase. This can be justified by understanding that as the Lyapunov exponent along the oscillator trajectory is 0 , then phase perturbations accumulate so this is like a first order dynamical process with a long relaxation time. As the the quantity $\xi(t)$ is stochastic it is called a Langevin force and the equation (2.27) a Langevin equation.

In Physics the principle of equipartition of energy assigns an energy due to thermal fluctuations of $\frac{1}{2} k T$ in each dimension. This is equated to the kinetic energy of the particle $\frac{1}{2} m\left\langle v^{2}\right\rangle$, where $\left\langle v^{2}\right\rangle$ denotes the ensemble average velocity. For a small mass $m$ the thermal velocity $v_{t h}=$ $\sqrt{\left\langle v^{2}\right\rangle}=\sqrt{\frac{k T}{m}}$ may be observable. The thermal motion of a particle is known as Brownian motion. 
The prototype Langevin equation is:

$$
\dot{u}=-\beta u+A(t)+K(r, t),
$$

where $u$ is velocity, $\beta$ is damping, $A(t)$ is the force due to noise, and $K(r, t)$ other forces affecting the noise process, including energy fields and external forcing functions. The Langevin equation (2.28) without a potential field is then:

$$
\dot{v}+\gamma v=\xi(t),
$$

with $<\xi(t) \xi\left(t^{\prime}\right)>=2 \gamma^{2} D \delta\left(t-t^{\prime}\right)$. The autocorrelation is solved for by multiplying (2.29) by $v\left(t^{\prime}\right)$, where $t^{\prime}=t+\tau$, as the mean of $v(t)$ is zero. By integrating factor then,

$$
\left\langle v(t) v\left(t^{\prime}\right)\right\rangle=\gamma D e^{-\gamma\left|t-t^{\prime}\right|},
$$

for large $t, t^{\prime}$. Let the particle displacement be $x(t),(v=\dot{x})$. Then the mean-square value of the displacement at time $t$ is given by:

$$
\begin{aligned}
<\left(x(t)-x_{0}\right)^{2}>=<\left[\int_{0}^{t} v\left(t_{1}\right) \mathrm{d} t_{1}\right]^{2} & >=<\int_{0}^{t} v\left(t_{1}\right) \mathrm{d} t_{1} \int_{0}^{t} v\left(t_{2}\right) \mathrm{d} t_{2}>, \\
& =\int_{0}^{t} \int_{0}^{t}<v\left(t_{1}\right) v\left(t_{2}\right)>\mathrm{d} t_{1} \mathrm{~d} t_{2} .
\end{aligned}
$$

Further, $\int_{0}^{t} \int_{0}^{t} \mathrm{e}^{-\gamma\left|t_{1}-t_{2}\right|} \mathrm{d} t_{1} \mathrm{~d} t_{2}=\frac{2}{\gamma} t-\frac{2}{\gamma^{2}}\left(1-\mathrm{e}^{-\gamma t}\right)$, therefore for large $t, t^{\prime}$ :

$$
<\left(x(t)-x_{0}\right)^{2}>=2 D t,
$$

(H. Risken,[14]).

Alternatively as acceleration is neglected,

$$
<v(t) v\left(t^{\prime}\right)>\approx \frac{1}{\gamma^{2}}<\xi(t) \xi\left(t^{\prime}\right)>=2 D \delta\left(t-t^{\prime}\right),
$$

leading to the same result. This justifies the interpretation of $D$ as a diffusion constant.

In a physical system the diffusion constant is a coefficient of transport for a second order spatial derivative $\frac{\partial^{2} P}{\partial x^{2}}$ giving the evolution in time $\frac{\partial P}{\partial t}$ of 
the concentration of particles wrt $x$ in a concentration gradient. In the current application it is the net effect of a number of random noise impulses on the phase probability distribution with time. The second order spatial derivative is the result of determining the net transport $f(x, t)$ at a plane $x$ from the difference in transports at $x \pm \Delta x$. Generally the Langevin equation (2.28) needs to be solved to find $\phi(u ; \Delta u)$ the transition probability that $u$ changes by $\Delta u$ in time $\Delta t$. This is carried out from stochastic principles and the transition probability substituted in the Fokker-Planck equation which expresses the dynamics of the statistics of a Markov process.

Beginning with the Chapman-Kolmogorov equation:

$$
W(u, t+\Delta t)=\int W(u-\Delta u, t) \phi(u-\Delta u ; \Delta u) \mathrm{d}(\Delta u),
$$

where $W(u, t)$ is the distribution of velocity $u$ (三 frequency $\frac{d \theta}{d t}$ ) at time $t$ and $\phi(u ; \Delta u)$ is the transition probability that $u$ changes by $\Delta u$ in time $\Delta t$ derived from a Langevin equation. This supposes that the system parameters are stable over $\Delta t$, so can be treated as constants, that is $u$ can be treated as a constant in calculating the acceleration, but the noise force term $A(t)$ fluctuates appreciably. That is to say a Markov process as the transition probability is independent of the dynamics of the system. For the Fokker-Planck equation the transition probability is found from the Langevin equation as $\Delta u=-\beta u \Delta t+B(\Delta t)$, where $B(\Delta t)=\int_{t}^{t+\Delta t} A(\xi) \mathrm{d} \xi$ is the net acceleration a Brownian particle undergoes in time $\Delta t$. Physically the distribution must tend to the Maxwellian temperature distribution of an ideal gas, as time goes to infinity, so

$$
w(B(\Delta t)) \rightarrow\left(\frac{1}{4 \pi q \Delta t}\right)^{\frac{3}{2}} \exp \left(-\frac{\mid B(\Delta t))\left.\right|^{2}}{4 q \Delta t}\right),(t \rightarrow \infty),
$$

where $q=\frac{\beta k T}{m}$.

The following lemma is useful.

Lemma 2.7.1. Let

$$
R=\int_{0}^{t} \phi(\xi) A(\xi) \mathrm{d} \xi
$$


then the probability distribution of $R$ is given by:

$$
w(R)=\frac{1}{\left(4 \pi q \int_{0}^{t} \phi^{2}(\xi) d \xi\right)^{\frac{3}{2}}} \exp \left(\frac{-|R|^{2}}{4 q \int_{0}^{t} \phi^{2}(\xi) \mathrm{d} \xi}\right) .
$$

This is derived from the requirement for the distribution to be the Maxwellian temperature distribution as $t \rightarrow \infty$, see Chandrasekhar [15].

So from the Langevin equation (2.28),

$$
\phi(u ; \Delta u)=\frac{1}{(4 \pi q \Delta t)^{\frac{3}{2}}} \exp \left(-\frac{|\Delta u+\beta u \Delta t|^{2}}{4 q \Delta t}\right),
$$

as the Langevin equation relates the change in velocity and the velocity expressed in the relaxation term, to the acceleration due to noise in the form required by the lemma (2.7.1). Clearly if the velocity is $u$ at $t=0$ then it is so with probability 1 , and the lemma (2.7.1) is applied over $t=\Delta t$.

In the case of simple Brownian motion of the phase without a potential field ( $\omega=0), \phi(\Delta u)=\phi(-\Delta u)$. Expanding $W$ and $\phi$ in (2.34) in Taylor series around $t$ and $u$ leads to:

$$
\begin{array}{r}
W(u, t)+\frac{\partial W}{\partial t} \Delta t=W(u, t) \int_{-\infty}^{\infty} \phi(\Delta u) \mathrm{d} \Delta u+\frac{\partial W}{\partial u} \int_{-\infty}^{\infty} \Delta u \phi(\Delta u) \mathrm{d} \Delta u, \\
+\frac{\partial^{2} W}{\partial u^{2}} \int_{-\infty}^{\infty} \frac{\Delta^{2}}{2} \phi(\Delta u) \mathrm{d} \Delta u
\end{array}
$$

which is the Fokker-Planck equation. $\int_{-\infty}^{\infty} \phi(\Delta u) \mathrm{d} \Delta u=1$ and the first order spatial term vanishes because the transition probability $\phi$ is even, leading to $\frac{\partial W}{\partial t}=D \frac{\partial^{2} W}{\partial u^{2}}$, where $D=\frac{q}{\beta^{2}}$, which gives $D=\frac{k T}{m \beta}$ in physical systems. With initial condition $W\left(u, t ; u_{0}\right) \rightarrow \delta\left(u-u_{0}\right),(t \rightarrow 0)$, the solution is:

$$
W\left(u, t ; u_{0}\right)=\frac{1}{(4 \pi D t)^{\frac{1}{2}}} \mathrm{e}^{\frac{-\left|u-u_{0}\right|^{2}}{4 D t}},
$$

which says that the initial probability given by a delta function diffuses with a Gaussian distribution. 
From the Langevin equation (2.28) $u-u_{0} \mathrm{e}^{-\beta t}=\mathrm{e}^{-\beta t} \int_{0}^{t} \mathrm{e}^{\beta \xi} A(\xi) \mathrm{d} \xi$. So taking $\phi(\xi)=\mathrm{e}^{\beta(\xi-t)}$ therefore from lemma (2.7.1),

$$
W\left(u, t ; u_{0}\right)=\frac{1}{(4 \pi D t)^{\frac{1}{2}}} \exp \left(\frac{-\left|u-u_{0}\right|^{2}}{4 D t}\right), D=\frac{q}{\beta^{2}} .
$$

The distribution of $x$ can be obtained in the same manner,

$$
x-x_{0}=\int_{0}^{t} u(\xi) d \xi \Rightarrow x-x_{0}=\int_{0}^{t}\left\{u_{0} \mathrm{e}^{-\beta \eta}+e^{-\beta \eta} \int_{0}^{\eta} \mathrm{e}^{\beta} \xi A(\xi) \mathrm{d} \xi\right\} \mathrm{d} \eta .
$$

This leads to the form:

$$
x-x_{0}-\beta^{-1} u_{0}\left(1-e^{-\beta t}\right)=\int_{0}^{t} \phi(\xi) A(\xi) \mathrm{d} \xi,
$$

where $\phi(\xi)=\beta^{-1}\left(1-\mathrm{e}^{\beta(\xi-t)}\right)$. Applying lemma (2.7.1),

$W\left(x, t ; x_{0}, u_{0}\right)=\left[\frac{m \beta^{2}}{2 \pi k T\left[2 \beta t-3+4 \mathrm{e}^{-\beta t}-\mathrm{e}^{-2 \beta t}\right.}\right]^{\frac{1}{2}} \exp \left(-\frac{m \beta^{2}\left|x-x_{0}-u_{0}\left(1-\mathrm{e}^{-\beta t}\right) / \beta\right|^{2}}{2 k T\left[2 \beta t-3+4 \mathrm{e}^{-\beta t}-\mathrm{e}^{-2 \beta t}\right]}\right)$,

or

$W\left(x, t ; x_{0}\right) \simeq(4 \pi D t)^{-\frac{1}{2}} \exp \left(-\frac{\left|x-x_{0}\right|^{2}}{4 D t}\right),\left(t \gg \beta^{-1}\right) \Rightarrow\left\langle\left(x-x_{0}\right)^{2}\right\rangle=2 D t, \quad\left(D=\frac{k T}{m \beta}\right)$.

For the case where $\Delta t \gg \tau_{\text {corr }} \gg \beta^{-1}$ the Langevin equation is not necessary as the noise dynamics are ineffective, and the method can be directly applied to the phase space $x$ independent of the velocity $u$. Therefore,

$$
W(x, t+\Delta t)=\int W(x-\Delta x, t) \phi(\Delta x) \mathrm{d}(\Delta x) .
$$

The transition probability is given by:

$$
\phi(\Delta x)=\frac{1}{(4 \pi D \Delta t)^{\frac{1}{2}}} \exp \left(-\frac{|\Delta x|}{4 D \Delta t}\right), D=\frac{q}{\beta^{2}}=\frac{k T}{m \beta},
$$

as this is derived from above with $\tau_{\text {corr }} \gg \beta^{-1}$ and $t=\Delta t \gg \tau_{\text {corr }}$. Therefore $\frac{\partial W}{\partial t}=D \nabla_{x}^{2} W$. For $\beta \gg 1$ the Langevin equation is heavily damped 
and the acceleration can be ignored which is equivalent to the previous case.

For oscillator noise the frequency of the oscillator has a noise term added. The probability distribution of the phase over time is required. The effect of the frequency on the phase noise can be modelled as the potential field term of a linear oscillator $\omega^{2} x$ not to be confused with the actual deterministic dynamics of the oscillator. It is seen that where the effect of the noise causes a phase deviation $> \pm \pi$ this causes a $2 \pi$ phase jump and the noise process is now effective in the adjacent oscillator cycle. So the force term due to the potential field is added to the noise dynamics to express this effect. Mathematically the potential force field term cancels out the damping so that the acceleration becomes effective so the equation is now that of a damped forced linear oscillator.

The Langevin equation therefore has the potential force field term $K(x, t)=$ $\omega^{2} x$ added and is analysed as an overdamped second order equation. Write

$$
\frac{\partial u}{\partial t}=-\beta u+A(t)-\omega^{2} x \Rightarrow \frac{\partial^{2} x}{\partial t^{2}}+\beta \frac{\partial x}{\partial t}+\omega^{2} x=A(t) .
$$

The homogeneous equation has for solution $x=a_{1} \exp \left(\mu_{1} t\right)+a_{2} \exp \left(\mu_{2} t\right)$ where $\mu_{i}$ are the roots of $\mu^{2}+\beta \mu+\omega^{2}=0$. Proceeding using variation of parameters, $a_{1}$ and $a_{2}$ are functions of time which satisfy:

$$
\exp \left(\mu_{1} t\right)\left(\frac{\mathrm{d} a_{1}}{\mathrm{~d} t}\right)+\exp \left(\mu_{2} t\right)\left(\frac{\mathrm{d} a_{2}}{\mathrm{~d} t}\right)=0
$$

and

$$
\mu_{1} \exp \left(\mu_{1} t\right)\left(\frac{\mathrm{d} a_{1}}{\mathrm{~d} t}\right)+\mu_{2} \exp \left(\mu_{2} t\right)\left(\frac{\mathrm{d} a_{2}}{\mathrm{~d} t}\right)=A(t) .
$$

Thus the solutions are

$$
x+\frac{1}{\mu_{1}-\mu_{2}}\left[\left(x_{0} \mu_{2}-u_{0}\right) \exp \left(\mu_{1} t\right)-\left(x_{0} \mu_{1}-u_{0}\right) \exp \left(\mu_{2} t\right)\right]=\int_{0}^{t} A(\xi) \psi(\xi) \mathrm{d} \xi,
$$

and

$u+\frac{1}{\mu_{1}-\mu_{2}}\left[\mu_{1}\left(x_{0} \mu_{2}-u_{0}\right) \exp \left(\mu_{1} t\right)-\mu_{2}\left(x_{0} \mu_{2}-u_{0}\right) \exp \left(\mu_{2} t\right)\right]=\int_{0}^{t} A(\xi) \phi(\xi) \mathrm{d} \xi$, 
where

$$
\psi(\xi)=\frac{1}{\mu_{1}-\mu_{2}}\left[\exp \left[\mu_{1}(t-\xi)\right]-\exp \left[\mu_{2}(t-\xi)\right]\right]
$$

and

$$
\phi(\xi)=\frac{1}{\mu_{1}-\mu_{2}}\left[\mu_{1} \exp \left[\mu_{1}(t-\xi)\right]-\mu_{2} \exp \left[\mu_{2}(t-\xi)\right]\right] .
$$

The lemma (2.7.1) is extended for 2 random variables corresponding to $x$ and $u$, see Chandrasekhar [15]. This allows the distribution functions $W\left(x, t ; x_{0}, u_{0}\right), W\left(u, t ; x_{0}, u_{0}\right)$, and $W\left(x, u, t ; x_{0}, u_{0}\right)$ to be determined in terms of the integrals, $\int_{0}^{t} \psi^{2}(\xi) \mathrm{d} \xi, \int_{0}^{t} \phi(\xi) \mathrm{d} \xi$, and $\int_{0}^{t} \phi(\xi) \psi(\xi) \mathrm{d} \xi$. The over damped case corresponds to $\beta_{1}=\left(\frac{1}{4}^{2}-\omega^{2}\right)_{\frac{1}{2}}$ real, giving for example:

$$
\begin{aligned}
& W\left(x, t ; x_{0}, u_{0}\right)=\left[\frac{1}{4 \pi D \int_{0}^{t} \psi^{2}(\xi) d \xi}\right]^{\frac{1}{2}} \times \\
& \exp \left(-\frac{\left(x-x_{0} \mathrm{e}^{-\frac{\beta_{1}}{2}}\left[\cosh \left(\frac{1}{2} \beta_{1} t\right)+\frac{\beta}{\beta_{1}} \sinh \left(\frac{1}{2} \beta_{1} t\right)\right]-\frac{2 x_{0}}{\beta_{1}} \mathrm{e}^{-\frac{\beta_{1}}{2}} \sinh \left(\frac{1}{2} \beta_{1} t\right)\right)^{2}}{\frac{2 \beta}{\omega^{2}} D\left\{1-\mathrm{e}^{-\beta_{1}}\left(\frac{2 \beta^{2}}{\beta_{1}^{2}} \sinh ^{2}\left(\frac{1}{2} \beta_{1} t\right)+\frac{\beta}{\beta_{1}} \sinh \left(\beta_{1} t\right)+1\right)\right\}}\right) .
\end{aligned}
$$




\section{Chapter 3}

\section{Coupled Oscillators}

\subsection{Introduction}

Consider the system:

$$
\ddot{x}+c^{2} x=\left(c^{2}-1\right) \sin (t) .
$$

This is an undamped oscillator driven by a periodic forcing function. The system is nonautonomous but can be made autonomous by introducing an additional dependent variable $\theta$ for $t$. The phase space is now $\mathbb{R}^{3}$ rather than $\mathbb{R}^{2}$. As $\theta$ is the argument of a periodic function it can be considered to lie in the interval $[0,2 \pi)$. Let $y=\dot{x}$. With initial conditions $x(0)=0, y(0)=$ $2 c+1, \theta(0)=0$, the solution of the system is:

$$
\begin{aligned}
& x(t)=\sin (t)+\sin (c t), \\
& y(t)=\cos (t)+c \cos (c t), \\
& \theta(t)=t \bmod 2 \pi .
\end{aligned}
$$

The phase space is the infinite slab $\mathbb{R}^{2} \times[0,2 \pi)$. As 0 is identical with $2 \pi$ the $\theta$ component can be seen to map onto a circle so that a trajectory that reaches $2 \pi$ in the $\theta$ component continues on from $\theta=0$. $x$ and $y$ are also periodic. There are two cases. If 1 and $c$ are commensurate, that 
is $c$ is rational, then the trajectory is a periodic orbit. If $c$ is $\frac{p}{q}$ then for $t=q \cdot 2 n \pi, c t=p \cdot 2 n \pi$ and the trajectory has period $q$. The trajectory lies on a torus, the exact path depending on the initial conditions. As $p$ turns through the torus are made for $q$ rotations of the torus, the rotation number of the trajectory is defined to be $\frac{p}{q}$. As the $x$ and $y$ variables of the equations given describe an ellipse they can be expressed in radial co-ordinates.

Let $\phi$ be the angle of the turn around the torus, that is in the latitudinal direction. If 1 and $c$ are incommensurate then $c$ is irrational. The orbit of the trajectory is then called quasi-periodic and is dense on the torus as the trajectory never returns to the initial conditions. The surface of the torus is 2 dimensional. The Poincaré-Bendixson theorem gives the possible invariant sets for a bounded flow in 2 dimensional space. These include equilibrium point, limit cycle, and saddle point with homoclinic orbit. So the dynamics of two oscillators correspond to uniform flow in a rectangle, that is periodic in the plane $\mathbb{R}^{2}$. As the flow is defined on a rectangle, it can be mapped onto a $T^{2}$ torus. Consider a Poincaré section of the torus at any angle $\theta$ in the longitude of the torus at time $t$. Assuming the flow on the torus has no fixed points this section defines a Poincaré map. Let $\phi(t)$ of the trajectory be $\phi_{n}$ and $\theta+2 \pi$ at time $t^{\prime}$ defines $\phi_{n+1}$ as $\phi\left(t^{\prime}\right)$.

\subsection{Nonisochronous driven oscillator}

\subsubsection{Introduction}

For an oscillator system driven by a fixed frequency signal, let $\tau$ be the period of the driving function in units of the intrinsic period of the oscillator system ( $\equiv \frac{p}{q}$ in the example). As the section of a torus is topologically equivalent to a circle the map:

$$
\phi_{n+1}=f\left(\phi_{n}, \tau\right),
$$


is known as a circle map. Expressing the map as:

$$
\phi_{n+1}=g\left(\phi_{n}\right)+\tau,
$$

makes the effect of the driving function explicit, $\tau$ corresponds to the forcing period in units of phase of the nominal frequency of the oscillator. For a periodically forced system the Poincaré map is identical to a time- $T$ map and this allows $\tau$ to be defined in terms of the driven system so that the map is autonomous. The map:

$$
\phi_{n+1}=\phi_{n}+\tau+b \sin \left(2 \pi \phi_{n}\right),
$$

known as the sine map, has been proposed as a prototype for periodically forced nonlinear oscillators [16]. Trignometrically the multiplication of two sine waves gives sum and difference terms. The sum term is discarded as it is periodic and synchronous with the difference term when the frequencies are rationally related and so averages out, and as the two solutions are superimposed it does not affect the synchronisation, which leaves the requisite function of phase difference driving synchronisation. The multiplication can be viewed as an elementary nonlinearity justifying the sine map as a generic prototype map.

An example of E. M. Zaslavsky [16] requires high dissipation to exhibit a sine map. He presents a perturbed nonlinear oscillator system with a stable limit cycle using action $I$, and angle $\vartheta$ variables. Let,

$$
\dot{I}=-\gamma\left(I-I_{0}\right)+\epsilon q(I, \vartheta) f(t), \quad \dot{\vartheta}=\omega(I),
$$

where $\epsilon$ is the perturbation parameter, $\gamma$ is the dissipation parameter, $I_{0}$ is the action which corresponds to the stable limit cycle if $\epsilon=0$ and $f(t)$ is $f(t)=\Sigma_{n} \delta(t-n T)$. Suppose that $q(I, \vartheta)=I_{0} \cos (\vartheta), \quad \omega(I)=\omega_{0}[1+$ $\left.\alpha \frac{\left(I-I_{0}\right)}{I_{0}}\right]$. Integrating and obtaining the mapping between two consecutive 
$\delta$-pulses, gives:

$$
\begin{aligned}
y_{n+1}= & \mathrm{e}^{-\Gamma}\left(y_{n}+\epsilon \cos \left(2 \pi x_{n}\right)\right) \\
x_{n+1}= & \left\{x_{n}+\frac{\Omega}{2 \pi}+\left(\frac{\alpha \Omega}{2 \pi \Gamma}\right)\left(1-\mathrm{e}^{-\Gamma}\right) y_{n}\right. \\
& \left.+\left(\frac{K}{\Gamma}\right)\left(1-\mathrm{e}^{-\Gamma}\right) \cos \left(2 \pi x_{n}\right)\right\} \bmod 1,
\end{aligned}
$$

where $y=\frac{\left(I-I_{0}\right)}{I_{0}}, \quad x=\frac{\vartheta}{2 \pi}, \quad \Omega=\omega_{0} T, \quad \Gamma=\gamma T$, and $K=\frac{\alpha \in \Omega}{2 \pi}$. If $\Gamma \gg 1$, the auxilary variable $y_{n}$ dynamics are nulled and only the $x_{n}$ angle variable is effective. The oscillator is then isochronous. The $x_{i}$ map is now:

$$
x_{n+1} \approx\left\{x_{n}+\frac{\Omega}{2 \pi}+\frac{K}{\Gamma} \cos \left(2 \pi x_{n}\right)\right\} \bmod 1 .
$$

The use of the sine map as a prototype map can be justified by considering the state variables of the oscillator in radial coordinates $r, \theta$. The forcing will perturb both $r$ and $\theta$. But assuming the system is isochronous, vector addition of the components of sinusoidal forcing leads to the $\sin \left(2 \pi \phi_{n}\right)$ dependence of the change in phase of the oscillator for each rotation of the forcing, or in the case just considered a cosine map, where the perturbation is vertically orientated as opposed to horizontally orientated for the sine map. So the Poincaré plot of a forced Poincare (2D) oscillator will be a 1D circle map. For a non-chaotic system a perturbation of amplitude will have little effect anyway as the -ve Lyapunov exponent causes the amplitude to quickly converge to the limit cycle.

As the trajectories do not fold, for small $b$ the map (3.4) is 1-1 and invertible of topological degree 1 . Clearly for $b=0$, there is no coupling and as the measure of the rational numbers is zero it is improbable that a periodic orbit will exist. Where $0<b<\frac{1}{2 \pi}$ the oscillator system is forced in a simple manner by the driving function. V.I.Arnold [17] in 1963, described a plot of the phase locking zones as a function of $b$ and $\tau$. These show a characteristic structure of zero width at rational values of $\tau$ on the $b=0$ horizontal axis, and widening as $b$ increases, and are known as Arnold tongues. The dynamics of the regions between the tongues are quasiperi- 
odic. For $b>\frac{1}{2 \pi}$ the map is no longer one-to-one and invertible, and the simple Arnold tongue structure is destroyed.

\subsubsection{Weak Coupling}

Perez and Glass [18] analyse the sine map numerically. The Arnold tongue structures for $b<\frac{1}{2 \pi}$ can be found by considering the effect on the fixed points of the sine map of changing the period $\tau$ of the driving function. It is seen that $\tau$ shifts the sine map up and down in the $\phi_{n+1} \operatorname{vs} \phi_{n}$ plot. At a critical value of $\tau$ dependent on the coupling the sine map will be tangent to the $\phi_{n+1}=\phi_{n}$ line. This is a tangent bifurcation point and corresponds to the edge of an Arnold tongue. As $\tau$ is decreased the bifurcation point divides into an unstable fixed point and a stable fixed point. These fixed points correspond to synchronisation of the oscillator with the driving function and the phase of the synchronisation corresponds to the phase of the fixed point in the sine map. As $\tau$ decreases further another tangent bifurcation of the sine map occurs coinciding with the opposite edge of the Arnolds tongue and desynchronisation.

\subsubsection{Quasiperiodic behaviour}

For the quasiperiodic behaviour between the tongues analysis is more difficult. V. I. Arnold [17] proved the existence of quasiperiodic solutions on the torus. Denjoy's Theorem states that if a $C^{2}$ orientation preserving diffeomorphism of the circle has the irrational rotation number $\mu$ then it is topologically equivalent to a rotation of the circle by $2 \pi \mu$. There are counterexamples for $C^{1}$ diffeomorphisms. Arnold proved that where a diffeomorphism $A: y \mapsto y+2 \pi \mu+a(y)$ of the circle with rotation number $\mu$ where $\mu$ is irrational, and where $a(y)$ is a $2 \pi$ periodic analytic function, then this diffeomorphism is analytically equivalent to a rotation by $2 \pi \mu$.

Let $\Im$ be the rotation by angle $2 \pi \mu$, and $H$ the desired diffeomorphism 
converting the rotation into $A$, then:

$$
\begin{array}{rll}
S^{1} & \stackrel{A}{\rightarrow} & S^{1} \\
H \uparrow & & \uparrow H \\
S^{1} & \stackrel{\Im}{\rightarrow} & S^{1}
\end{array} \text { that is } \quad H \cdot \Im=A \cdot H .
$$

Let $H(z)=z+h(z), h(z+2 \pi)=h(z)$ then $h(z+2 \pi \mu)-h(z)=a(z+h(z))$, by operating $H$ on $S^{1}$ and the rotation $z+2 \pi \mu$ of $S^{1}$, and equating $2 \pi \mu+$ $a(z+h(z))$ with the difference. Approximately $h(z+2 \pi \mu)-h(z)=a(z)$ as $A$ differs only a little from a rotation so $a(z)$ is small and $h(z)$ must be at least as small, so the $h(z)$ difference in the $a(z)$ argument is expressed in a 2nd order term. Solve this equation by expanding the known function $a$, and $h$ in a Fourier series:

$$
a(z)=\Sigma a_{k} \mathrm{e}^{i k z}, \quad h(z)=\Sigma h_{k} \mathrm{e}^{i k z}
$$

equating coefficients $\Rightarrow h_{k}=\frac{a_{k}}{\left(\mathrm{e}^{2 \pi i k \mu}-1\right)}, h(z)$ exists (converges) as Arnold proved that although the denominator $\left(\mathrm{e}^{2 \pi i k \mu}-1\right)$ might be quite small as every irrational number $\mu$ admits rational approximations $\frac{p}{q}$ with error $\left|\mu-\frac{p}{q}\right|<\frac{1}{q^{2}}$ for arbitarily large $q$, he found that $\left|\mu-\frac{p}{q}\right| \geq \frac{K}{|q|^{2+\sigma}}$ held also for appropriate $K, \sigma>0$ and for any integers $p, q \neq 0$. This leads to $\left|\mathrm{e}^{2 \pi i k \mu}-1\right| \geq \frac{K}{2|k|^{1+\sigma}},|K|>0$, so that arbitrarily small denominators exist only on a set of measure 0 . Thus contrary to notions of structural stability, quasiperiodic solutions with irrational rotation numbers are preponderant for small $\varepsilon$.

\subsubsection{Structure of Arnolds tongues}

For the circle map $f\left(\phi_{n}, \tau\right)$, define the rotation number $\sigma$. Let $\Delta \phi_{i}=$ $f\left(\phi_{i}, \tau\right)-\phi_{i}$. Then the rotation number is:

$$
\sigma=\lim _{N \rightarrow \infty} \sup \frac{1}{N} \sum_{i=1}^{N} \Delta \phi_{i} .
$$


A fixed point of period $N$ exists if $\phi_{N}=\phi_{0}(\bmod 1) ; \phi_{i} \neq \phi_{0}$, for $i=$ $1,2, \ldots, N-1$, where the $\phi_{i}$ are generated by iterating the circle map $f\left(\phi_{i}, \tau\right)$. For a cycle of period $N$ there is a phase locking in the ratio $N: M$ where $M=\phi_{N}-\phi_{0}$, and $\sigma=\frac{M}{N}$. The stability of the cycle is given by the condition $\prod_{i=0}^{N-1}\left|\left(\frac{\partial f}{\partial \phi_{i}}\right)_{\phi_{i}}\right|<1$. If an extremum of $f$ is on a cycle the cycle is superstable. The locus of superstable cycles on the $b$ vs $\tau$ diagram are called the skeletons of the phase-locking zones as this corresponds to a boundary of a phase locking zone.

Let $g\left(\phi_{i}\right)$ of a circle map have a winding number of one. Then $g\left(\phi_{i}+\right.$ $j)=g\left(\phi_{i}\right)+j$ where $j$ is an integer. This leads to a translational symmetry for the Arnolds tongues. If there is $N: M$ phase locking for a given value of $\tau$ then for $\tau^{\prime}=\tau+p$ where $p$ is an integer there is $N:(M+p M)$ phase locking. Further if $g(x)$ is odd and if there is stable $N: M$ phase locking for an initial condition $x_{0}$ and $\tau=p+\epsilon$ then there will also be stable $N:((2 p+1) N-M)$ phase locking for an initial condition of $-x_{0}$ and $\tau^{\prime}=p+1-\epsilon$. If the Poincare map is monotonic $\left(b<\frac{1}{2 \pi}\right)$ then the rotation number is a monotonic function of $\tau$, independent of the initial condition and piecewise constant on the rationals. Therefore Arnolds tongues corresponding to all the rationals exist.

What is the structure of the Arnolds tongues for $b<\frac{1}{2 \pi}$ ? The Arnolds tongues of order $n$ form a Farey sequence of order $n$. This is defined as the reduced fraction $\frac{a}{b}: 0 \leq \frac{a}{b} \leq 1,1 \leq b \leq n$. The sequence $F_{n}$ may be generated inductively from $F_{n-1}$ by repeating the fractions in sequence and inserting $\frac{a+a^{\prime}}{b+b^{\prime}}$ between $\frac{a}{b}$ and $\frac{a^{\prime}}{b^{\prime}}$ if $\left(b+b^{\prime}\right) \leq n$. Geometrically each fraction is the terminal point of a line to the origin that does not cross another point in a box of size $n$, as in figure(3.1). For two fractions $\frac{a}{b}, \frac{c}{d}$ in sequence $a c-b d=1$. The geometry can be used to prove number theories about the approximation of irrational numbers with rational numbers which are at the base of Arnold's proof of the existence of quasiperiodic solutions such as the following theorem.

Theorem 3.2.1. For any irrational number $\mu$, there exist arbitrarily accurate ra- 
tional approximations whose error is less than the reciprocical value of the square of the denominator,

$$
\left|\mu-\frac{p}{q}\right|<\frac{1}{q^{2}} .
$$

Proof. Let $e_{k}$ and $e_{k-1}$ lie on different sides of $y=\mu x$ where $e_{i}$ is a rational number $\frac{p_{i}}{q_{i}}$. Let $e_{-1}=(1,0), e_{0}=(0,1)$. Construct $e_{k+1}=e_{k-1}+a_{k} \cdot e_{k}$, where $a_{k}$ is a positive integer so that $e_{k+1}$ is on the same side of $y=\mu x$ as $e_{k-1}$. $\exists a_{i}>0$ as $e_{i}$ is based at the origin and has a gradient $\neq \mu$ so based on $e_{i-1}$ it does not cross $y=\mu x$ as the error of $e_{i-1}$ is greater than the error of $e_{i}$, and $a_{i}$ is chosen to minimise the error with $\mu x$. The parallelogram spanned by $e_{k+1}, e_{k}$ has oriented area $e_{k+1} \times e_{k}=(-1)^{k}$ inductively as $e_{0} \times e_{-1}=-1$. Taking the difference between two successive fractions $\frac{p_{k}}{q_{k}}-\frac{p_{k+1}}{q_{k+1}}=\frac{(-1)^{k}}{q_{k} q_{k+1}}$ implies $\left|\mu-\frac{p}{q}\right|<\frac{1}{q^{2}}$ as $y=\mu x$ lies between two successive fractions and $q_{k+1}>q_{k}$.

The fraction $\frac{p_{k}}{q_{k}}$ can be expressed as a continued fraction $a_{0}+\frac{1}{a_{1}+\frac{1}{a_{2}+\frac{1}{\ldots+\frac{1}{a_{k-1}}}} .}$

Further, for any $\varepsilon>0$, there exists $C>0$ such that for any integers $p, q>0, \quad\left|\mu-\frac{p}{q}\right|<\frac{C}{|q|^{2+\varepsilon}}$. See V.I. Arnold [19].

\subsubsection{Strong coupling}

What is the effect of the Poincare map being non-monotonic, $\left(b \geqslant \frac{1}{2 \pi}\right)$ ? For $b>\frac{1}{2 \pi}$ each Arnold tongue structure for $b<\frac{1}{2 \pi}$ divides into two branches which can intersect. Dynamics include bistability, period doubling bifurcations, and chaos. If $g\left(x_{i}\right)$ is of degree (winding number) 1 with a single minimum $x_{\min }$ and maximum $x_{\max }$ in the interval. Let $x_{i}=H_{i}(\tau)$ where $H_{i}(\tau)$ is the $i$ th iteration of $g\left(x_{i}\right)+\tau$ from $x_{0}=x_{\max }$. As $g\left(x_{i}\right)$ is of degree 1 , so that $g\left(x_{i}-1\right)=g\left(x_{i}\right)-1, H_{q}(j)-H_{q}(j-1)=q$. There will be a 


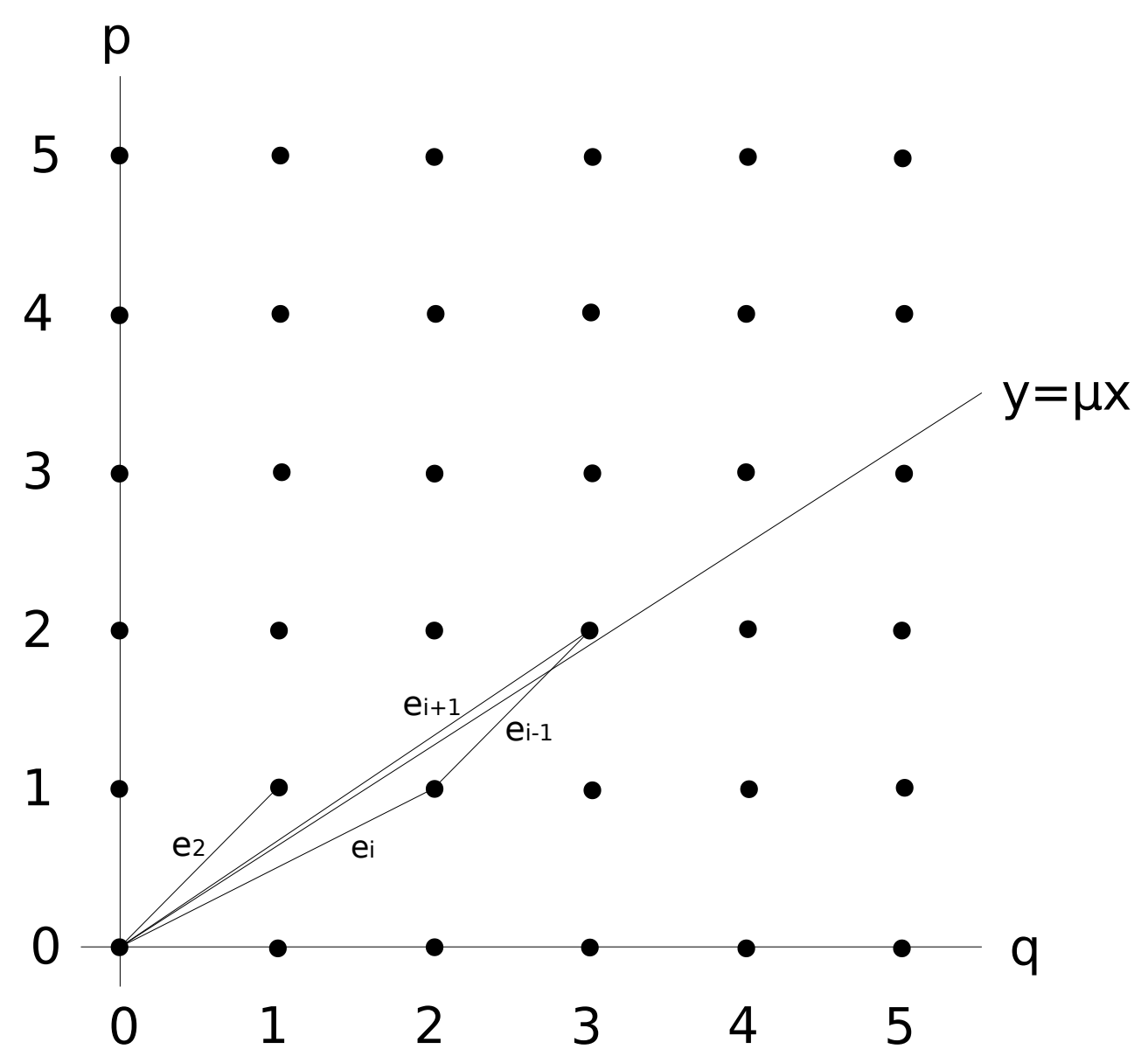

Figure 3.1: construction of a Farey sequence 
superstable cycle for each value of $\tau$ for which $x_{0} \bmod 1=H_{q}(\tau) \bmod 1$. Since $H_{q}(\tau) \bmod 1=x_{0} \bmod 1$ at least $q$ times as $\tau$ varies from $j-1$ to $j$ there are at least $q$ superstable cycles occuring at $q$ different values of $\tau$, with $q$ different rotation numbers. Similarly for $x_{\text {min }}$. Therefore there exist at least 2 values of $\tau$ corresponding to boundaries of a locking region for each rational rotation number. For the sine map the locking regions overlap and there is bistability where the boundaries of different locking regions intersect as $\tau$ varies.

For the sine map also sucessive curves of period doubling bifurcations are found as $b$ increases, leading to chaotic dynamics as the sequence of period doubling bifurcations accumulates at a limit point. This same bifurcation structure appears to be duplicated as a continuation of each Arnold tongue in the region $b<\frac{1}{2 \pi}$, as $b$ increases. The regions overlap so multiple ratios are possible for some ranges of the parameters of coupling and detuning $\tau$.

An example of P. Coullet et al.[20] shows the applicability of the sine map to a second order nonautonomous system where the coupling is strong so that the map is no longer a diffeomorphism, and also the use of maps on an annulus for analysing the perturbations of limit cycles of nonlinear systems. Consider the second order nonautonomous equation:

$$
\ddot{x}+\left(x^{2}-\sigma\right) \dot{x}+\omega_{1}^{2} x+x^{3}+\lambda \cos \left(\omega_{2} t\right)=0 .
$$

This describes a forced van der Pol-Duffing type oscillator. The $(x, \dot{x})$ plane is mapped onto itself by the flow of the equation over $\frac{2 \pi}{\omega_{2}}$ units of time to give a Poincaré map. Some values of $\sigma$ and $\lambda$ show folding of the annulus region containing the limit cycle under the effect of the time $t=\frac{2 \pi}{\omega_{2}}$ map given by the system.

Consider an injective mapping from the annulus onto itself which models the folding phenomenon. Let the annulus A be bounded by $r=1$ and 


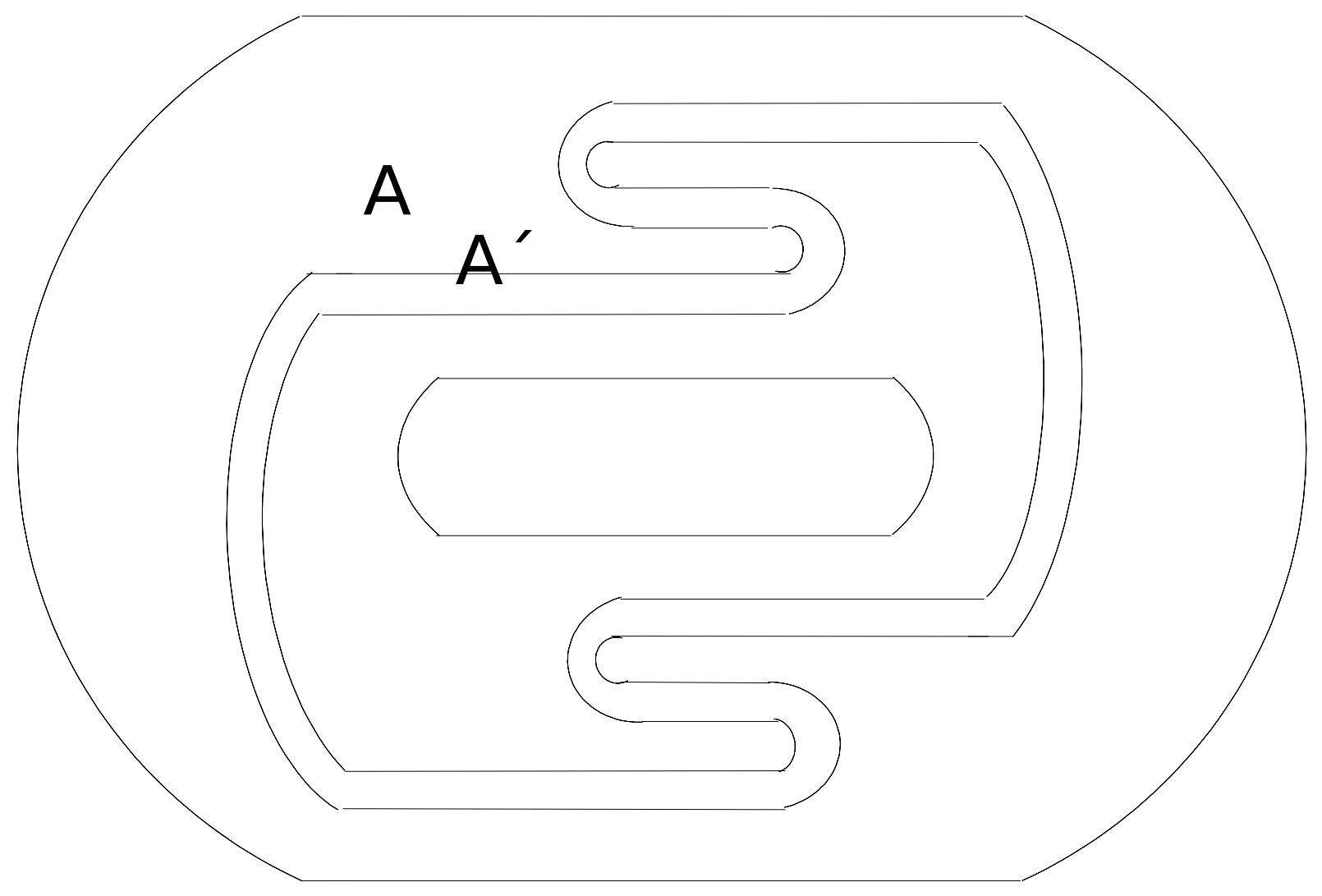

Figure 3.2: annulus region containing oscillator limit cycle and effect of mapping showing horseshoe formation, indicating chaotic dynamics 
$r=\frac{1}{3}$. Set $\rho=r-\frac{2}{3}$. The injective map $T$ on $A$ is defined by:

$$
\begin{aligned}
T: \theta^{\prime} & =\theta+\varphi+\epsilon \sin (p \theta)+\beta \rho \bmod 2 \pi, \\
\rho^{\prime} & =\alpha[\rho+\sin (p \theta)],
\end{aligned}
$$

where $p$ is an integer, $\varphi$ a given angle, and $\alpha<\frac{1}{4}, \beta=\frac{(q-1)}{p}+\epsilon, 0<q<1$. When $\alpha \rightarrow 0, T$ reduces to a map of the circle onto itself:

$$
\tilde{T}: \theta^{\prime}=\theta+\varphi+\epsilon \sin (p \theta) \bmod 2 \pi .
$$

When $\epsilon<\frac{1}{p}, \tilde{T}$ is a diffeomorphism of the circle, and $T(A)$ does not reveal any folding. When $\epsilon>\frac{1}{p}, \tilde{T}$ is a noninvertible map and $T(A)$ shows the folding phenomenon.

\subsection{Dynamics of phase locking}

\subsubsection{Deterministic isochronous oscillators}

What are the dynamics of the phase locking? Pikovsky et al.[21] consider an autonomous dissapative dynamic system with a stable limit cycle in its phase space. The phase $\phi$ is defined as corresponding to motion along the direction of the limit cycle. This is the direction of the zero Lyapunov exponent, motion transverse to the limit cycle corresponding to the negative Lyapunov exponents. The phase of the oscillation can be defined away from the limit cycle. Isochronic surfaces of constant phase may depend on the displacement from the limit cycle. If there is no dependence on displacement then the surfaces intersect the limit cycle at $\frac{\pi}{2}$. This is the simple phase model of coupled oscillators where the state variables consist simply of the phases of the oscillators. If there is a dependence then $\phi=\phi(\theta, A)$ is a function of the displacement $A$, and $\theta$ is a radial angle coordinate geometrically related to the limit cycle. As the relaxation times of perturbations in amplitude are finite whereas the Lyapunov exponent of 
the phase is zero, the dynamics of the phase can be expressed in a single equation:

$$
\frac{d \phi}{d t}=\omega_{0}+\varepsilon Q(\phi, \varphi)
$$

where $\omega_{0}$ is the free running frequency of the oscillator, $\varepsilon$ is a small parameter coupling the driving force to the oscillator phase, $\varphi$ is the phase of the driving function, and $Q$ is $2 \pi$ periodic in both $\phi$ and $\varphi$ expressing the effect of the driving function on the oscillator. Taking $Q$ modulo $2 \pi$ in both parameters describes dynamics on a torus. The Poincare map in $\varphi$ is a circle map $\phi_{n+1}=\phi_{n}+\varepsilon F\left(\phi_{n}\right)$, and $F$ is periodic in $2 \pi$.

The phenomenon of $n: m$ synchronisation is defined in terms of a fixed relationship between the phases of the oscillator and the driving function, or as phase locking. In the case of $1: 1$ synchronisation the phases would be locked when the phases of the oscillators coincide exactly, that is $\phi=\varphi$ but more generally a constant phase difference is possible so that $\phi=\varphi+$ constant. In both cases the phase of the oscillator is rotating uniformly with the frequency of the driving function. Inspection of (3.16) shows that both these cases where $\frac{d \phi}{d t}=\omega$ ( $\omega$ is the forcing frequency), require the driving function $Q$ to be a function of the phase difference as the phase locking condition requires that the difference in phases is a constant, so that $Q(\phi, \varphi) \equiv q(\phi-\varphi)$. Therefore let $\psi=\phi-\varphi$, the phase difference so that equation (3.16) becomes:

$$
\frac{d \psi}{d t}=\omega_{0}-\omega+\varepsilon q(\psi) .
$$

This equation has an equilibrium point if the frequency mismatch (detuning) is small enough ( $\omega_{0}$ is the nominal frequency of the oscillator not the driven frequency), so $\varepsilon q_{\min }<\omega-\omega_{0}<\varepsilon q_{\max }$, to determine the synchronisation region in the $(\omega, \varepsilon)$ plane. In the synchronisation region the phase difference remains constant $\psi=\delta$ depending on the detuning of the oscillator and $\delta=q^{-1}\left(\left(\omega-\omega_{0}\right) / \varepsilon\right)$.

In general $Q(\phi, \varphi)$ is not a function of the phase difference $\psi$ and the phase $\phi$ of the oscillator does not rotate uniformly with the frequency of 
the driving function. Even where the frequencies are rationally related the phase difference is not a constant but fluctuates in a bounded manner. Therefore phase locking is defined to occur when the phase difference lies between bounds so that $|\phi-\varphi-\delta|<$ constant. For general $n: m$ synchronisation the phase difference is taken to be $n \phi-m \varphi$. A weaker condition is frequency locking. The rotation number corresponds to the ratio between the observed frequency $\Omega$ and the frequency of the external force $\omega$ so, $\sigma=\frac{\Omega}{\omega}$. If $\sigma$ is rational $\Omega=\frac{m}{n} \omega$ and this corresponds to $m: n$ synchronisation. This definition is useful if the progression of phase is not uniform as $\Omega=<\dot{\phi}>$ the average of the instantaneous frequency $\dot{\phi}$.

If the phase is not well defined, synchronisation can be characterised by means which do not depend explicitly on phase. The mean frequency of a chaotic oscillation can be calculated simply using a Poincaré surface by $\omega=\lim _{t \rightarrow \infty} 2 \pi \frac{N_{t}}{t}$, where $N_{t}$ is the number of crossings of the Poincaré surface in time $t$. The method can be simply applied to a time series and in the most elementary case $N_{t}$ can be found by counting the maxima of $x(t)$. The phase of a chaotic oscillator can be considered to be uniformly distributed across an ensemble of $N$ systems. The ensemble average of an observable $u$ can be taken so $U(t)=\frac{1}{N} \sum_{1}^{N} u_{i}$. The non-coherent contributions cancel so their amplitude decreases at a rate $N^{-\frac{1}{2}}$. If the systems are driven coherently by an external force the oscillations become apparent in $U(t)$ as they are coherent. The ensemble average can also be related to the time dependent probablity distribution function of amplitude $W\left(u, t-t_{0}\right)=W\left(u, t+T-t_{0}\right)$ when the system is forced periodically. The ensemble average in the limit $N \rightarrow \infty$ can be expressed as $U\left(t-t_{0}\right)=\int u W\left(u, t-t_{0}\right) d u$. The variance of the oscillations $V=\overline{(U-\bar{U})^{2}}$ serves as a measure of phase synchronisation where the bar means in the limit $t \rightarrow \infty$, or over the interval $t=T$ where the probability distribution is periodic.

Another indirect aspect of the phase dynamics is the frequency spectrum of the observable $u(t)$. If the phase of a chaotic system is locked 
by a periodic force then the signal becomes highly correlated, so $u(t)$ and $u(t+T)$ differ only by the chaotic amplitude as the phases are similar. The autocorrelation function $C(\tau)=<u(t) u(t+\tau)>$ has a periodic nature revealed as $\tau \rightarrow \infty$, with maxima at $\tau=n T$. Thus the power spectrum shows peaks at the frequency of the driving force $\Omega$ and its harmonics which are not present if the system is not synchronised. Therefore the intensity of the power spectrum is used to indicate synchronisation. This is calculated via the Wiener lemma

$$
S=\lim _{t \rightarrow \infty} \frac{1}{t} \int_{0}^{t} C^{2}(\tau) d \tau .
$$

A resonance peak in the curve of $S$ vs $\Omega$ shows the phase synchronisation.

\subsubsection{Example of forced oscillator}

Pikovsky et al. [21] consider a periodically forced weakly non-linear oscillator as an example. Let,

$$
\ddot{x}-\alpha \dot{x}+\gamma x^{2} \dot{x}+\omega^{2} x=\phi \cos \Omega t .
$$

This is the forced Van der Pol oscillator. Notice that in comparison to the unforced equation the solution can vary in phase as well as amplitude and therefore quadrature amplitude terms in $\cos \Omega t$ and $\sin \Omega t$ are needed. Therefore substitute as the solution $x=a(t) \mathrm{e}^{i \Omega t}$, the complex exponential representation, where the amplitude $a(t)$ is complex and the actual solution is $\operatorname{Re}\left(a(t) \mathrm{e}^{i \Omega t}\right)$. Therefore $i \Omega \dot{a}+\left(-\Omega^{2}-i \alpha \Omega+i \gamma \Omega|a|^{2}+\omega^{2}\right) a-\phi=0$, using $\dot{a} \ll a$ to neglect terms in $\dot{x}$ and $\ddot{x},\left(\left(\gamma\left|a^{2}\right|-\alpha\right) \approx 0\right.$ near the limit cycle), and using $|x|^{2}$ instead of $x^{2}$ so,

$$
\dot{a}=-i \nu a+\alpha a-\gamma|a|^{2} a-i \varepsilon,
$$

where $\nu=\frac{\Omega^{2}-\omega^{2}}{\Omega}$, the normalised detuning, and $\varepsilon=\frac{\phi}{\Omega}$. This is the variational equation for Van der Pol oscillator obtained by transforming the coordinate system, to be relative to the forcing frequency and averaging 
the oscillating terms. So the fixed points represent synchronisation to the driving oscillator, and the dynamics are $2 \mathrm{D}$ as the driving oscillator perturbs the amplitude as well as the phase of the Van der Pol oscillator.

Figures (3.3) and (3.4) show solutions of the Van der Pol variational equation for two sets of parameters. The vector field is shown by the arrows and the solutions from a range of initial conditions by the solid lines. Inspecting the 1-D complex phase plane of $a$, for small forcing $(\varepsilon<.6)$ and starting from the synchronous state $(v=0)$, a saddle point shown with an $\times$ on figure (3.3) and a stable fixed point shown with an $\circ$ lie on a closed orbit enclosing an unstable focus near the origin. So the solution is a constant amplitude sine wave at frequency $\Omega$. As $\nu$ is increased the saddle point and the stable fixed point move closer together along the orbit. The amplitude and the phase of the sine wave given by the vector to the fixed point change, equivalently the phase difference $\psi$ is increasing. The saddle point and the fixed point cancel as synchronisation is broken. $a$ moves around a closed orbit, equivalently the phase difference increases monotonically with slow periodic $2 \pi$ jumps. The sine wave shows changing phase with some modulation of amplitude as $|a|$ does not vary greatly.

For large $\varepsilon$ the loss of synchronisation is via the Andronov-Hopf bifurcation. For $\nu \approx 0$ the phase plane contains a stable fixed point focus so $a$ is a constant. As $\nu$ increases the fixed point becomes a focus so that the trajectories encircle the fixed point as they converge to it (see figure (3.4)). A limit cycle develops and $a$ moves around the limit cycle. Now the phase difference $\psi$ is oscillating but the synchronisation is maintained so there is amplitude and phase modulation of the frequency $\Omega$ sine wave.

The synchronisation is broken when the limit cycle encloses the origin and the phase difference $\psi$ now has $2 \pi$ jumps. The phase and amplitude modulation of the frequency $\Omega$ sine wave depends on the symmetry of the limit cycle around the origin and the movement of $a$ around the limit cycle. Holmes and Rand[22] describe the bifurcation set for the averaged Van der Pol equation in terms of the $(\nu, \varepsilon)$ plane, and describe the 2D planar phase 


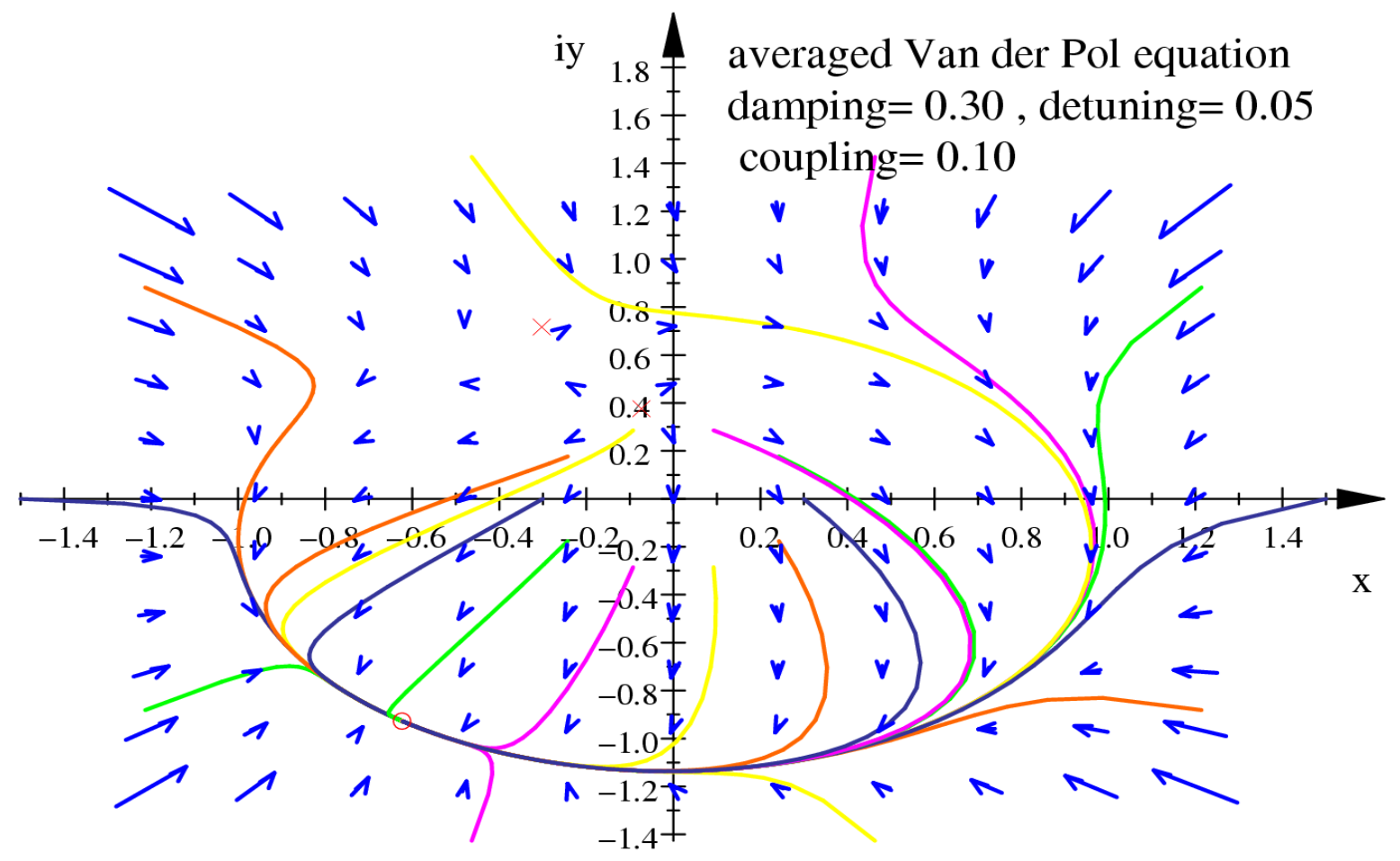

Figure 3.3: forced Van der Pol oscillator phase plane in locking region 


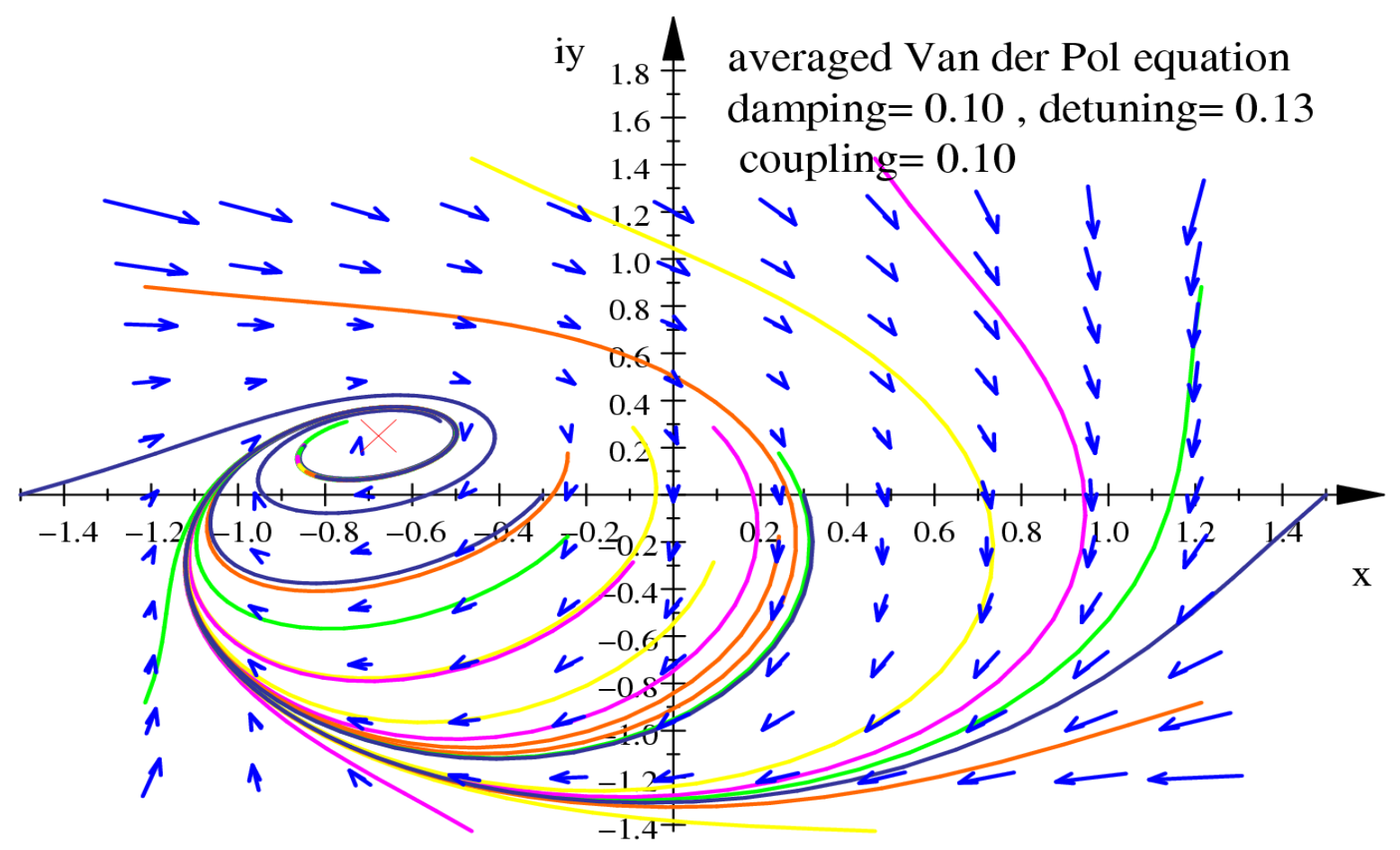

Figure 3.4: forced Van der Pol oscillator near boundary of locking region 
plots in regions of the bifurcation parameter plane.

\subsubsection{Chaotic oscillators}

For a chaotic oscillator there is a Lyanupov exponent $>0$. In the most general case where the system is nonisochronous the phase depends on the amplitude of the oscillation. The effect of a driving function is to add further dimensions to an autonomous system. Generalising the Poincaré map construction for a chaotic oscillator the dynamics can be represented as:

$$
\begin{aligned}
A_{n+1} & =T\left(A_{n}, \varphi_{n}\right), \\
\frac{d \phi}{d t} & =\omega\left(A_{n}, \phi, \varphi\right), \\
\frac{d \varphi}{d t} & =\Omega,
\end{aligned}
$$

in the case of periodic forcing, where $\varphi_{n}$ is a simple function of the driving frequency $\Omega$ and the return time for $A_{n}$. If the forcing amplitude is small and proportional to $\varepsilon$ then:

$$
\frac{d \phi}{d t}=\omega_{0}+\varepsilon G\left(A_{n}, \phi, \varphi\right)+F\left(A_{n}\right),
$$

so this is similar to a driven periodic oscillator with a noisy chaotic term added. Analogous to the construction for a chaotic oscillator (2.26), if $T$ is a perturbed chaotic map then this can express the effect on the phase of the effect of the driving function on the amplitude of the oscillator, through $F$, and $G$ reduces to a simple dependence on $\phi$ and $\varphi$.

A.S.Pikovsky et al. [9] give two examples of periodically driven chaotic oscillators. A Rössler attractor driven by $E \cos (\Omega t)$ is found to have clear phase-locking regions in the form of Arnold tongues, dependent on the amplitude $E$ and the frequency of the external force $\Omega$, at the fundamental resonance $\Omega \approx \omega_{0}$ and at the resonances $\Omega \approx 2 \omega_{0}$ and $2 \Omega \approx \omega_{0}$. It is noted that there appears to be no threshold of synchronisation and this 
is attributed to the high phase coherence of the Rössler system meaning that the chaotic noise term is small. For a Lorenz system phase-locking is found only at the main resonance $\Omega \approx \omega_{0}$ and only for sufficiently large amplitudes of external force. This is explained by a relatively large chaotic noise term.

The effect of noise is to diminish the phase-locked structures. This can be analysed by adding a noise term $\xi(t)$ to (3.16). For a system with a periodic forcing function,

$$
\frac{d \psi}{d t}=\omega_{0}-\omega+\varepsilon q(\psi)+\xi(t) .
$$

The noise is then driving the dynamics of the phase difference. The dynamics can be considered as that of an overdamped particle in a potential field as in (2.7),

$$
V(\psi)=\left(\omega-\omega_{0}\right) \psi-\varepsilon \int^{\psi} q(x) d x .
$$

The particle moves down a potential gradient corresponding to the effect of the difference in the frequencies of the two oscillators therefore and $\psi$ increases influenced by the noise. Often $q(\psi)=-\sin (\psi)$ and the effect is that the particle is in equilibrium at local minima corresponding to synchronisation. The particle will return to the minimum under the influence of the potential field as it is displaced by noise but the amplitude of the noise can be such that the particle is displaced to an adjacent minimum and this is seen as phase slips of $2 \pi$ in the synchronisation. Quantitatively the effect of the Langevin equation $\frac{d \psi}{d t}=\omega_{0}-\omega-\varepsilon \sin (\psi)+\xi(t)$, can be found stochastically through the Fokker-Planck equation, but direct solution of the Langevin equation is simpler when boundary conditions on the phase are not involved. 


\subsection{Perturbed Nonisochronous Oscillator}

\subsubsection{Introduction}

The general case of a coupled oscillator pair includes asymmetric coupling so that the dynamics of the phase difference are no longer autonomous but driven by higher dimensional dynamics. Clearly this could include an exponential transient, oscillation, chaos, or noise. A. Shilnikov et al. [23] overview the current understanding of the synchronisation of an oscillator in the plane. The use of 2D Poincaré maps in analysing coupled oscillator dynamics, and the disappearance of the saddle node bifurcation at the edge of an Arnold tongue are discussed. It is seen that the PoincaréBendixson theorem defines the possible dynamics in a plane and coupled with a driving oscillator defines a 3D system, and this motivates the system under consideration.

\subsubsection{Planar dynamics}

A typical system is given as an autonomous system with a single parameter, $\dot{x}=X(x, \mu)$ consisting of a stable periodic orbit $L_{\mu}$ which becomes a homoclinic loop to the saddle equilibrium point as $\mu \rightarrow 0^{+}$. The period of the limit cycle is of the order of $|\ln (\mu)|$. This a consequence of the slow dynamics on the limit cycle near the saddle point, and the validity of a linear approximation to the eigenvalues there. The saddle point has a significant effect on the map of the coupled system. The formation of a limit cycle can be seen to be consistent with the dynamics of the Van der Pol equation, $\ddot{x}-\mu\left(1-x^{2}\right) \dot{x}+\omega^{2} x=0$. At $\mu=0$ the equation is a conservative system in a parabolic potential well. As $\mu$ is increased from zero the system is no longer Hamiltonian and the limit cycle is established from a Hopf bifurcation at $\mu=0$. Normalising the period to one with the substitution $\tau=\omega t$ results in $\epsilon=\mu / \omega$ so the period is inversely proportional to $\mu$ for the perturbed Van der Pol oscillator. 
How do these dynamics arise? Let $\dot{x}=A x+f_{2}(x)+f_{3}(x)+\ldots$, where $A$ is an $n \times n$ constant matrix and $f_{i}(x)$ are homogeneous vector valued polynomials in $x$ of order $i$. The $f_{i}(x)$ are sums of terms of the form $x_{1}^{m_{1}} x_{2}^{m_{2}} \ldots x_{n}^{m_{n}}, m_{1}+\ldots+m_{n}=i, m_{i} \epsilon\{0\} \cup N$. There is a critical point at $x=0$. The Poincaré Normal Form Theorem says that this system can be transformed into the linear form $\dot{y}=A y$ by the transformation $x=y+h_{2}(y)+\ldots$, unless resonances occur, that is the eigenvalues of $A$, $\lambda_{i}, i=1 \ldots n$ are resonant with $\lambda_{i}=\sum_{j=1}^{n} m_{j} \lambda_{j}$ for some $i$. The nonresonant terms can be removed from the equation. This is called normalization. See V.I. Arnold[19]. Bendixson's criterion applies to a 2D system and states that for a planar autonomous system $\dot{x}=f(x)$ in a domain $D \subset \mathbb{R}^{2}$, the system can only have periodic solutions, that is a limit cycle or a homoclinic loop, if $\nabla \cdot\left(f_{1}, f_{2}\right) \equiv$ trace $(D f)$ changes sign or $=0$ in $D$. For a constant matrix $A$, with eigenvalues $\lambda_{1}, \lambda_{2}$, trace $(A)=\lambda_{1}+\lambda_{2}$.

\subsubsection{Hopf bifurcation}

An example is the Hopf bifurcation. Let a single parameter system be given by $\dot{x}=A(\mu) x+f(\mu, x)$. $f$ has quadratic and higher terms in $x$. The case where $A(\mu)$ has purely imaginary eigenvalues for some values of $\mu$ as is the case for the Van der Pol equation $\ddot{x}+\omega^{2} x=\mu\left(1-x^{2}\right) \dot{x}$ is the Hopf bifurcation. For $\mu>0$ a limit cycle exists. More generally, let

$$
\begin{aligned}
& \dot{x}=\mu x-\omega y+\ldots, \\
& \dot{y}=\omega x+\mu y+\ldots,
\end{aligned}
$$

where the higher order terms are at least quadratic and higher, $\omega \neq 0$. If $\mu=0$ the eigenvalues of the linear part are purely imaginary. Normalisation removes all quadratic terms and some cubic terms. To degree 3 the system becomes:

$$
\begin{aligned}
& \dot{u}=d \mu u-(\omega+c \mu) v+a\left(u^{2}+v^{2}\right) u-b\left(u^{2}+v^{2}\right) v+\ldots \\
& \dot{v}=(\omega+c \mu) u+d \mu v+b\left(u^{2}+v^{2}\right) u+a\left(u^{2}+v^{2}\right) v+\ldots
\end{aligned}
$$


In polar coordinates:

$$
\begin{aligned}
& \dot{r}=\left(d \mu+a r^{2}\right) r+\ldots, \\
& \dot{\theta}=\omega+c \mu+b r^{2}+\ldots
\end{aligned}
$$

This shows a pitchfork bifurcation in $r$ at $\mu=0$ which corresponds to a Hopf bifurcation in the full system.

\subsubsection{Global bifurcations}

A saddle point can be seen to be the consequence of a higher order potential well. Consider the cubic potential $V(x)=x^{3}-a^{2} x$. Let a Hamiltonian be $H(x, y)=y^{2}+V(x)$. Then the resulting conservative system has a homoclinic orbit.

Higher order potential functions have more elaborate homoclinic orbits. So the double well Duffing equation with a potential function $V(x)=$ $\left(x^{2}-a^{2}\right)^{2}=x^{4}-2 a^{2} x^{2}+a^{4}$ has a two homoclinic orbit vector field from the saddle point. So this is the simplest system with a homoclinic orbit. For an area preserving or Hamiltonian system in the plane, linearization of hyperbolic fixed points gives trace $(D f) \equiv 0$, so all fixed points are saddle points or centers, no sinks or sources can exist. These dynamics are therefore typical of a large class of conservative systems.

A tangent node may be perturbed in a single parameter map where the gradient is +1 or equivalently where the eigenvalue is zero in a flow. They are therefore associated with oscillators.

The global bifurcations which occur with perturbations such as the crossing of unstable and stable manifolds and periodic cycles create complex dynamics and are therefore of interest. The simplest global bifurcation for planar vector fields occur where a trajectory joins two saddle points or forms a loop on a saddle point. Let $\dot{x}=f(x, \mu)$ be a one parameter planar vector field with a homoclinic loop on a saddle point. Let $p_{0}$ be the saddle point with trace $\left(D f\left(p_{0}\right)\right)<0$ such that at $\mu=\mu_{0}$ there exists a homoclinic loop $\gamma_{0}$. 
A Poincaré return map $P_{\mu}$ is constructed at a point $q \neq p_{0}$ on the stable manifold of $p_{0}$ (far enough away from $p_{0}$ that the system is no longer linear), along a line segment $M$ transversal to $q$. Only points on the inside of the stable manifold are studied as for trace $\left(D f\left(p_{0}\right)\right)<0$ and $\mu>\mu_{0}$ the unstable manifold leaves the region of the saddle point and the stable manifold. As trace $\left(D f\left(p_{0}\right)\right)<0$ the saddle point is contracting, flow lines near $p_{0}$ are attracted to the homoclinic loop $\gamma_{0}$ as they flow past $p_{0}$, whereas for the rest of $\gamma_{0}$ the divergence must be near zero.

Let the eigenvalues of $p_{0}$ be $-\alpha, \beta$ with $\alpha>\beta>0$, and use the stable and unstable manifolds as $x$ and $y$ coordinates. Let $\delta$ be such that $1>\delta>$ $\frac{\beta}{\alpha}$. Therefore in an $\varepsilon$-neighbourhood of $p_{0}$,

$$
\left|\frac{d y}{d x}\right|=\left|\frac{\beta y+\ldots}{-\alpha x+\ldots}\right|<\delta\left|\frac{y}{x}\right| .
$$

Consider a trajectory starting near the stable manifold at $x=\varepsilon, y=y_{0}<$ $\varepsilon$. Then using a Gronwall estimate of the trajectory, at $y=\varepsilon, x=x_{1}<$ $y_{0}^{\frac{1}{\delta}}(\varepsilon)^{1-\frac{1}{\delta}}$. As the divergence is small away from the saddle point this is the Poincare return map on the transversal $M$. Therefore as $x \rightarrow q$ along $M$, the slope of $P_{\mu}(x)$ approaches zero. At $\mu=\mu_{0}, q=P_{\mu_{0}}(q)$ as at $\mu_{0}$ the stable and unstable manifolds coincide in a homoclinic orbit. So $P_{\mu_{0}}$ touches $y=x$. As $\mu$ increases $>\mu_{0}, P_{\mu}$ intersects $y=x$ implying the existence of a periodic orbit.

Therefore a homoclinic loop from a saddle point has a stable periodic cycle associated with it and the effect of the bifurcation can be usefully studied as periodic forcing will cause chaotic homoclinic entanglement.

\subsubsection{Dynamics near a Saddle point}

The system is supposed to be able to be written in the form:

$$
\begin{gathered}
\dot{x}=\lambda x+f(x, y, \mu), \\
\dot{y}=\gamma y+g(x, y, \mu),
\end{gathered}
$$


analytic, with a $-v e$ saddle value $\rho=\lambda+\gamma<0$ so the saddle point is contracting meaning that the system is dissipative. S. Wiggins [24] shows that this is a consequence of $i$ ) translating the saddle point to the origin, ii) utilising the linear stable and unstable eigenvalues as coordinates, iii) utilisation of the stable and unstable manifolds as coordinates.

Coupled dynamics are expressed as a perturbation of this system. The perturbed system is:

$$
\begin{gathered}
\dot{x}=\lambda x+f(x, y, \mu)+\mu p(x, y, t, \mu), \\
\dot{y}=\gamma y+g(x, y, \mu)+\mu q(x, y, t, \mu),
\end{gathered}
$$

where $p$ and $q$ are $2 \pi$ periodic in $t$. It is seen that the system is a coupled oscillator system when $p$ and $q$ depend on $x$ and $y$. The effect of the perturbation is to add a dimension in the $\theta$ coordinate. The saddle point is perturbed from the origin. The stable and unstable manifolds no longer coincide. When the mainfolds traverse they do so a countable number of times at homoclinic points where the dynamics simultaneously satisfy the constraints of both the unstable and stable manifolds. Geometrically a homoclinic tangle of the manifolds is formed.

Whereas for the 1D forced oscillator it is convenient to make a Poincare plot at a cross section of the toroid, which leads to the 1D circle map, for the dynamics of a 2D forced oscillator the Poincare section is made in the $x$ plane at a small distance $\delta$ from the saddle point at the origin on the stable manifold and likewise for the $y$ coordinate on the unstable manifold.

The Poincare plot is the composition of the dynamics near the saddle point and near the homoclinic loop. This leads to a 2D map in $\theta$ and $y$. Now $\theta$ can be interpreted as the phase difference between the oscillators as the limit cycle returns periodically through the $x$ plane at $x=\delta$. It is seen therefore that the Poincare map thus constructed is a $2 \mathrm{D}$ annulus and equivalent to the annulus map containing the limit cycle of the oscillator as the $y$ coordinate corresponds to variations in the limit cycle radius. A 
Poincaré map $T_{\mu}$ can be modelled as:

$$
\begin{aligned}
& \bar{y}=[y+\mu(1+f(\theta))]^{\nu}, \\
& \bar{\theta}=\theta+\omega-\frac{1}{\gamma} \ln [y+\mu(1+f(\theta))],
\end{aligned}
$$

where $\nu=-\left(\frac{\lambda}{\gamma}\right)>1, \omega$ is a constant, and $\mu(1+f(\theta))$ is the Melnikov function with $\langle f(\theta)\rangle=0$. It is seen that the $y$ map is obtained from the composition of the subharmonic Melnikov function on the periodic orbit and the relation $y=x^{-\nu}$ near the saddle point, and the $\theta$ map from the transit time of the trajectory near the saddle point where the dynamics are controlled by the linear system.

It is also seen then that the period is of order $|\ln (\mu)|$, and $\rightarrow \infty$ as $\mu \rightarrow 0$, as the perturbation from the manifold is of order $\mu$, and transit time derived from the exponential dynamics of the linear system between the planes at $x=\delta$, and $y=\delta$. The limit set of the map $T_{\mu}$ for small $\mu$, lies in an annulus $K_{\mu}=\left\{0<y<C \mu^{\nu}, 0 \leq \theta<2 \pi\right\}$, for some $C>0$.

The equations (3.29), (3.30) may be rescaled with $y \rightarrow \mu^{\nu} y$ to give the form:

$$
\begin{array}{r}
\bar{y}=[1+f(\theta)]^{\nu}+\ldots, \\
\bar{\theta}=\theta+\bar{\omega}-\frac{1}{\gamma} \ln [1+f(\theta)]+\ldots,
\end{array}
$$

where $\bar{\omega}=\left(\omega-\frac{1}{\gamma} \ln \mu\right) \rightarrow \infty$, as $\mu \rightarrow 0^{+}$. It is seen therefore that the Poincaré map is dominated by the family of circle maps. Differentiating the $\theta$ map, if

$$
\frac{1}{\gamma} \frac{f^{\prime}(\theta)}{1+f(\theta)}<1
$$

the map $T_{\mu}$ does not have an extremum point, so it has no folds in it and is invertible. It is found that in this case the image of the annulus $K_{\mu}$, under $T_{\mu}$ is also an annulus bounded by two curves of the form $y=h_{ \pm}(\theta)$. The contraction results in a smooth invariant closed curve. 
For strong coupling the gradient of the map must be $-v e$ or sufficiently $+v e$ over an interval $I=\left[\theta_{1}, \theta_{2}\right]$. If $f^{\prime}(\theta)<0$ everywhere on $I$, then the gradient of the $\theta$ map is greater than 1 , and with the condition:

$$
\frac{1}{\gamma} \ln \left(\frac{1+f\left(\theta_{1}\right)}{1+f\left(\theta_{2}\right)}\right)>2 \pi(m+1), m \geq 2,
$$

ensures that as $\theta$ ranges from $\theta_{1}$ to $\theta_{2}$ the map image overlaps with the interval $I$ at least $m$ times. Alternatively, if

$$
\frac{1}{\gamma} \frac{f^{\prime}(\theta)}{1+f(\theta)}>2
$$

everywhere on $I$ then the gradient is less than -1 , and with the condition:

$$
\frac{1}{\gamma} \ln \left(\frac{1+f\left(\theta_{2}\right)}{1+f\left(\theta_{1}\right)}\right)>2\left(\theta_{2}-\theta_{1}\right)+2 \pi(m+1), m \geq 2,
$$

the interval $I$ is mapped onto itself $m$ times, so chaotic trajectories will exist in either case, as the construction is analogous to the Smale horseshoe.

\subsubsection{Bifurcations near a Saddle point}

Whereas for a plain limit cycle oscillator the sine map is used for a prototype map, now the influence of the saddle point on the limit cycle is given effect. In this case $f(\theta)=A \sin \theta$ leads to a map based on $\ln (1+A \sin \theta)$. It is seen from the criterion for the $\theta$ map gradient (3.33), that for $A<\frac{\gamma}{\sqrt{1+\gamma^{2}}}$, then the invariant closed curve is an attractor for all small $\mu$. From the criterion for the winding of the map (3.34), it is seen that there are complex dynamics for $A>\tanh 3 \pi \gamma$.

Shilnikov et al.[23] describe the structure of the resulting synchronisation zones, in parameters $A$ and $-\ln \mu$, as $A$ determines the coupling and $-\ln \mu$ the period. The zones are based on the axis $-\ln \mu$ at coordinates $(2 \pi k, 0)$ where $k$ is an integer. The upper boundary is the bifurcation curve of a saddle node which curves up to meet the axis $A=1$. The lower boundary is the bifurcation curve of a saddle node which curves to 
lower rotation numbers but does't terminate on the $A=1$ axis. Therefore it intersects with synchronisation regions of lower rotation numbers. The rotation numbers coexist in these intersecting regions.

Interior to the synchronisation region as $A$ increases the invariant curve breaks down in a period doubling bifurcation where the curve is no longer diffeomorphic to a circle. As the invariant curve consists of a saddle fixed point with the unstable manifolds closed on the stable fixed point of a saddle node bifurcation, it is this stable fixed point which bifurcates in a period doubling bifurcation, so becomes a saddle point connected to the stable fixed points of the period 2 cycle. The bifurcation curve follows the boundaries of the synchronisation region and is closest to the $-\ln \mu$ axis in the middle of the zone.

Along the boundaries of the zone the dynamics retain their integrity as $A$ increase until the region of complex dynamics is reached where the closed invariant curve ends with homoclinic touches of the unstable manifold of the saddle point with the stable manifold. Along the boundary of the zone before the region of complex dynamics the closed curve commences to develop wiggles as it converges to the saddle point, and the homoclinic touches occur at the boundary of the region of complex dynamics $(A>\tanh 3 \pi \gamma)$. See figure (3.5). In the interval between the small $A$ region and the complex dynamics the oscillation of the tangent vector to the unstable manifold causes it to connect to the saddle point nonsmoothly. Leaving the synchronisation zone for $A<\frac{\gamma}{\sqrt{1+\gamma^{2}}}$ the closed curve persists and the dynamics are quasiperiodic, or have a rational rotation number with a long period. On the boundary above this point the dynamics as the synchronisation zone is left are either intervals of chaotic and simple dynamics alternate nearer the small $A$ interval or chaotic near the region of complex dynamics. 


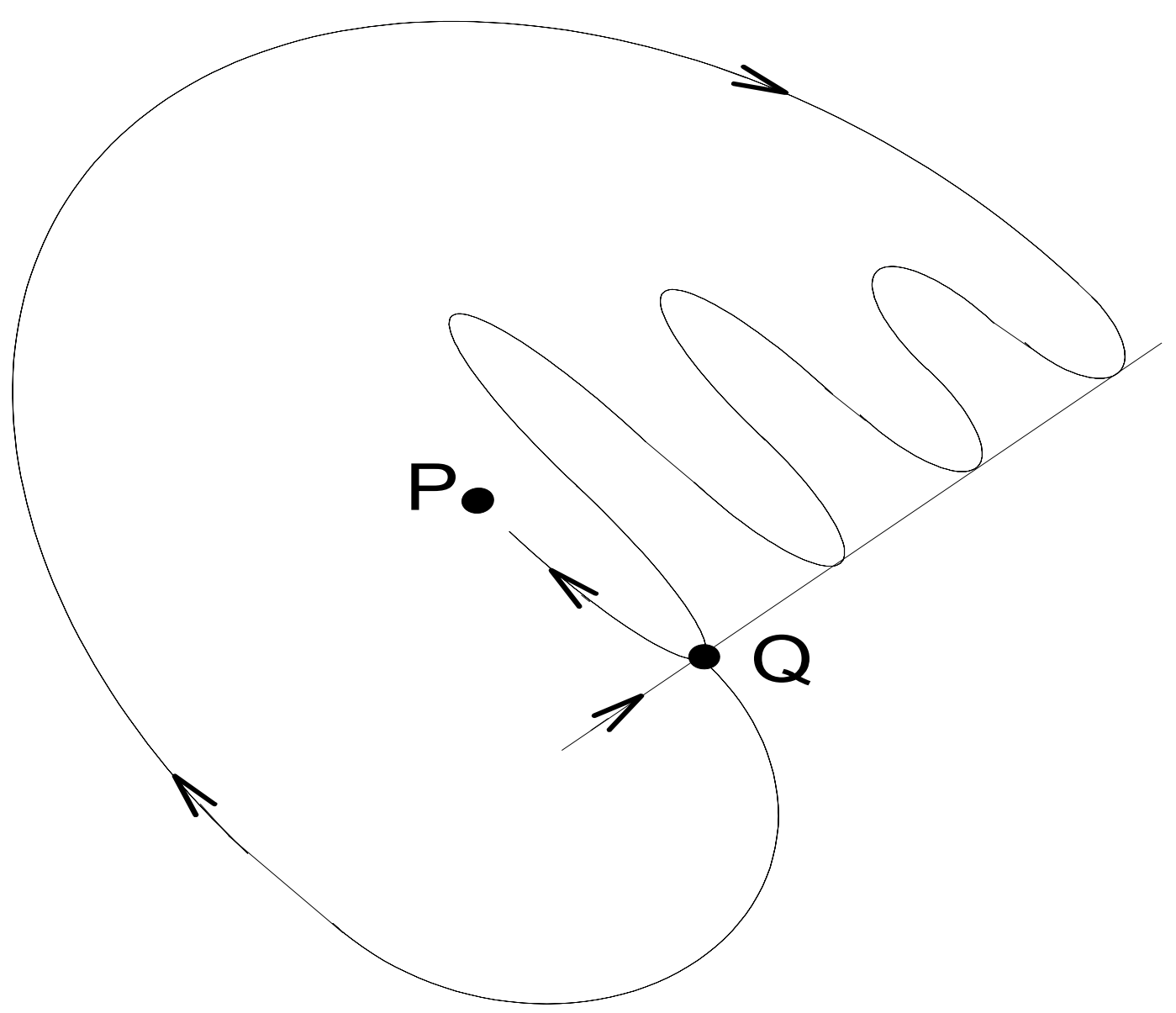

Figure 3.5: Homoclinic touches at boundary of locking zone, P stable fixed point, $\mathrm{Q}$ saddle point 


\subsection{Method of Averaging}

\subsubsection{Introduction}

The method of averaging is applied to solving perturbed systems where the motion of the unperturbed system occurs at a time constant much smaller than the effects of the perturbation. Originally Lagrange (1788) solved a problem $\dot{x}=\varepsilon f(x, t)$ by expanding $f(x, t)$ in a Fourier series and discarding the periodic terms retaining only the constant term. He studied planetary orbits by perturbing a system of $\mathrm{N}$ uncoupled oscillators. Subsequently Van der Pol (1934) used the method to analyse the Van der Pol oscillator. Lagrange used variation of parameters to solve a first order system $\dot{x}=A(t) x+\varepsilon g(t, x), x(0)=x_{0}$. For example $A(t)=\left(\begin{array}{cc}0 & 1 \\ -\omega^{2} & 0\end{array}\right)$ for the weakly nonlinear oscillator.

After [25], the solution of the homogeneous equation constitutes the fundamental matrix $\Phi(t)$. Substitute $x=\Phi(t) y$ giving:

$$
\dot{\Phi} y+\Phi \dot{y}=A(t) \Phi y+\varepsilon g(t, \Phi y) .
$$

Therefore,

$$
\Phi \dot{y}=\varepsilon g(t, \Phi y) .
$$

$y$ is solved for by inverting the fundamental matrix, so

$$
\dot{y}=\varepsilon \Phi^{-1}(t) g(t, \Phi(t) y),
$$

with initial values $y(0)=\Phi^{-1}(0) x_{0}$, revealing the problem of Lagrange $\dot{y}=\varepsilon f(t, y)$. Let $f(t, y)$ be periodic in $t$. Then $f(t, y)$ can be expressed as a Fourier series $g_{0}(y)+g_{1}(y) \sin t+h_{1}(y) \cos t+\ldots$. Supposing that $f(t, y)$ is averaged over a time longer than the period of the oscillation, then as $y$ varies in the time scale $\frac{1}{\varepsilon}$, the contribution from these terms is zero and the equation depends on $g_{0}(y)$. 


\subsubsection{Co-moving coordinates}

It is seen that the fundamental matrix for the Van der Pol equation is

$$
\left(\begin{array}{cc}
\cos \omega t & -\sin \omega t \\
-\omega \sin \omega t & -\omega \cos \omega t
\end{array}\right) .
$$

This establishes a frame of coordinates relative to the solution of the unperturbed system and is known in mechanics as the method of co-moving coordinates.

Generalising the method, the matrix

$$
\left(\begin{array}{cc}
\cos \left(\frac{\Omega t}{k}\right) & -\sin \left(\frac{\Omega t}{k}\right) \\
-\frac{\Omega}{k} \sin \left(\frac{\Omega t}{k}\right) & -\frac{\Omega}{k} \cos \left(\frac{\Omega t}{k}\right)
\end{array}\right),
$$

where $\Omega$ is the forcing frequency, $k$ an integer, and $\omega$ the natural frequency is $\approx \frac{\Omega}{k}$, is known as the Van der Pol transformation and establishes coordinates relative to the forcing frequency, and so gives a dynamical time $t=\frac{k}{\Omega}$ Poincaré plot.

\subsubsection{Averaging Theorem}

The averaged equation: $\dot{y}=\varepsilon \frac{1}{T} \int_{0}^{T} f(y, t, 0) d t \triangleq \varepsilon \tilde{f}(y)$ (where $f(y, t, \varepsilon)$ expresses higher order terms in $\varepsilon$ ), has an associated time $t=T$ flow map $P_{0}$. Similarly let the time $t=T$ flow map of the perturbed system be $P_{\varepsilon}$. The Averaging Theorem shows that a hyperbolic fixed point of $P_{\varepsilon}$ has a corresponding fixed point of the same stability type in $P_{0}$ as well as having solutions in unstable and stable manifolds corresponding to $O(\varepsilon)$. The time $t=T$ flow map approximates a Poincaré map on a differentiable manifold with a vector field restricted to a domain $D$. Thus the Poincaré map of the perturbed system $P_{\varepsilon \mid D}$ is topologically equivalent to $P_{0 \mid D}$ for $\varepsilon>0$ sufficiently small, as these types of behaviours are local.

This does not generally apply to global behaviour such as periodic orbits, and homoclinic bifurcations. For example a hyperbolic periodic orbit 
of averaged equations will correspond to an invariant closed curve in the Poincaré map $P_{\varepsilon \mid D}$. But the dynamics may be quite complex due to resonance effects and contain sets of periodic points as $\varepsilon \rightarrow 0$.

In the case where $f(x, t)$ is periodic in functions with incommensurate periods, then it is generally expressible as a finite sum $f(x, t)=\sum_{i=1}^{N} f_{i}(x, t)$ where $f_{i}$ is $T_{i}$-periodic in $t$. The averaged equation becomes:

$$
\dot{y}=\varepsilon \sum_{i=1}^{N} \frac{1}{T_{i}} \int_{0}^{T_{i}} f_{i}(y, t) d t, y(0)=x_{0} .
$$

\subsubsection{Validity of Averaging method}

For the validity of the method following the notation and explanation of V.I.Arnold [19], typically the unperturbed system is:

$$
\begin{aligned}
& \dot{\phi}=\omega(I), \\
& \dot{I}=0 .
\end{aligned}
$$

For example this can be seen to be the equation for dynamics on an $\mathrm{n}$ dimensional torus with $\mathrm{n}$ angular coordinates $\phi_{i}, i=1 . . n$. The dynamics of the $\phi_{i}$ are considered to be much faster than the dynamics of the $I_{i}$, so a perturbation of the $I_{i}$ variables is therefore of interest, as the perturbation of the $\phi_{i}$ variables averages out. If this system is perturbed in proportion to $\varepsilon$ then over times of order 1 the full solution is necessary, but over times of order $\frac{1}{\varepsilon}$ the dependence on $\phi_{i}$ can be averaged out.

Let the perturbed equations be:

$$
\begin{aligned}
\dot{\phi} & =\omega(I)+\varepsilon f(I, \phi, \varepsilon), \\
\dot{I} & =\varepsilon g(I, \phi, \varepsilon),
\end{aligned}
$$

where $f$ and $g$ are $2 \pi$ periodic in $\phi$. The averaged equation is therefore:

$$
\dot{J}=\varepsilon G(J),
$$

where $G(J)=\frac{\oint g(J, \phi, 0) d \phi}{\oint d \phi}$, the mean value of $g$ over the unperturbed ntorus. The presumption is that the solution of the $I$ component of the 
perturbed equation is close to the solution of the averaged equation over time $\frac{1}{\varepsilon}$. Consider a time period $1 \ll T \ll \frac{1}{\varepsilon}$ so this is an intermediate time period compared to the fast variables and the slow variables. The displacement of the projection of the perturbed trajectory on the base ( $I$ variables) is of the order $\varepsilon T \ll 1$. To a first approximation $I$ is a constant, $\varepsilon=0$, and $\phi$ given by the unperturbed equation, so

$$
\Delta I=\varepsilon T\left[\frac{1}{T} \int_{0}^{T} g(I, \phi(t), 0) d t\right]+o(\varepsilon T),
$$

as the expression in square brackets is the average velocity of the displacement over time $T$. This is nearly the time average of $g$ as $T \gg 1$. Let slow time $\tau=\varepsilon t$ so that $t=\frac{1}{\varepsilon}$ corresponds to $\tau=1$. So the motion of the slow variables occurs over the time scale $\frac{1}{\varepsilon}$. Then $\frac{\Delta I}{\Delta \tau} \approx$ time average of $g \approx \frac{\partial I}{\partial \tau}$, but $\frac{\partial J}{\partial \tau}=G(J)$ meaning that the time average of $g$ is determined from the space average of $g$ (over $\phi$ space), which is $G$.

Thus the averaging method is valid where the trajectories are uniformly distributed on the torus, that is quasi-periodic trajectories, and not near resonant trajectories where the frequencies are commensurate. Clearly the effect of the synchronization of frequencies is that the trajectories are no longer dense on the surface (wrt the nominal frequency difference) because of their stability in a locking region and the dimension of the n-torus is reduced. The space average, averages uniformly over the surface of the torus, whereas the time average covers the torus as a function of the length $T$ of the trajectory, except if it is resonant and then clearly the trajectory follows a lower dimensional path on the surface of the torus. And as long as $T \ll \frac{1}{\varepsilon}$ the approximation $g(I, \phi(t), 0)$ is valid for $g(I, \phi(t), \varepsilon)$ over the surface of the torus, as the second order terms in $\varepsilon$ remain insignificant, and the average serves for $G(J)$ as any trajectory therefore covers the torus in this time scale so variations in $\phi(t)$ (i.e. $\left.\varepsilon^{2} f()\right)$, are not significant. 


\subsection{Action/Angle coordinates}

\subsubsection{Hamiltonian oscillators}

Oscillatory dynamics can be described in terms of Hamiltonian theory by the perturbation of a Hamiltonian system. The Hamiltonian system is commonly expressed in action/angle coordinates $I / \phi$ as this simplifies the analysis. In this case the action variables vary slowly compared to the periodic angle variables. Let a Hamiltonian (conservative energy or volume preserving) system be expressed as:

$$
\begin{aligned}
& \dot{\phi}=\frac{\partial H}{\partial I}, \\
& \dot{I}=-\frac{\partial H}{\partial \phi},
\end{aligned}
$$

such that the unperturbed Hamiltonian $H_{0}$ depends only on $I$. The perturbed system is given by:

$$
H=H_{0}(I)+\varepsilon H_{1}(I, \phi, \varepsilon),
$$

where $H_{1}$ is $2 \pi$ periodic in $\phi$. The Hamiltonian equations are then:

$$
\begin{aligned}
& \dot{\phi}=\omega(I)+\varepsilon \frac{\partial H_{1}}{\partial I}, \\
& \dot{I}=-\varepsilon \frac{\partial H_{1}}{\partial \phi} .
\end{aligned}
$$

It is seen that in a Hamiltonian system with $n$ degrees of freedom and $n$ frequencies, that evolution of the slow variables does not occur as the averaged system has the form $\dot{J}=0$. This is a consequence of the periodicity of $H_{1}$ meaning that the integral of $\frac{\partial H_{1}}{\partial \phi}$ over a period is zero. The effect of this is that for Hamiltonian systems, usually the dynamics of the slow variables are invariant for long time frames. Phase curves starting initially between invariant tori remain there. In the case of 2 degrees of freedom the invariant tori divide the 3 dimensional Hamiltonian space into domains in which the phase curves are trapped. In the case of 3 or more degrees of 
freedom the invariant tori do not separate the Hamiltonian space and the phase curves can move slowly between the tori. This is known as Arnold diffusion.

\subsubsection{System action variables}

The notion of an adiabatic invariant is used to define the action variables of a Hamiltonian system. Quantities asymptotically preserved under sufficiently slow variations of the parameters of a Hamiltonian system are called adiabatic invariants.

Following Arnold [19]. Let $\dot{x}=v(x, \lambda)$ be a Hamiltonian system where $\lambda$ is a parameter (or a coefficient of a system). A function of $x$ and $\lambda$ is called an adiabatic invariant if for any sufficiently smooth function $\lambda(\tau)$ of the slow time $\tau=\varepsilon t$ (where $\varepsilon$ is the perturbation parameter), the variation of $I(x(t), \lambda(\varepsilon t))$ along a solution curve remains small in the time interval $0 \leq t \leq \frac{1}{\varepsilon}$, where $\varepsilon$ is small. i.e. $I(x, \lambda(\varepsilon t))=I(x(0), \lambda(0))+o(1)$ on the time scale $\frac{1}{\varepsilon}$. Having $\lambda(\tau)$ a function of slow time means to say that $\lambda(\tau)$ varies in slow time comparably to $x(t)$ in normal time. The system then becomes:

$$
\begin{aligned}
\dot{\phi} & =\omega(I, \lambda(\varepsilon t)), \\
\dot{I} & =0 .
\end{aligned}
$$

\subsubsection{Generating function}

Arnold introduces the idea of a generating function. For a Hamiltonian system, time independent, such that $\dot{p}=-\frac{\partial H}{\partial q}, \quad \dot{q}=\frac{\partial H}{\partial p}$ of size $n$ degrees of freedom and $2 n$ variables, then let $S(I, q)$ be a generating function to transform the coordinates $p, q \rightarrow I, \phi$. Then $p=\frac{\partial S}{\partial q}, \phi=\frac{\partial S}{\partial I}, H(p, q)=$ 
$H\left(\frac{\partial S}{\partial q}, q\right)=H_{0}(I)$. The Hamiltonian system becomes:

$$
\begin{aligned}
& \dot{I}=0, \\
& \dot{\phi}=\omega(I)=\frac{\partial H_{0}}{\partial I} .
\end{aligned}
$$

The implication of there existing a generating function $S$ and therefore of $I$ being a constant is that the Hamiltonian system is integrable in the sense that for an $n$ degrees of freedom Hamiltonian system, there exist $n$ integrals $F_{i}$, which are $i$ ) constants of motion, that is $\dot{F}_{i}=\left\{F_{i}, H\right\}=0$ and ii) functionally independent (can't be expressed as a function of the others) and iii) in involution, that is $\left\{F_{i}, F_{k}\right\}=0$ for $i, k=1 . . n$ where \{\} are the Poisson brackets $\left\{F_{i}, F_{k}\right\}=\frac{\partial F_{i}}{\partial q} \frac{\partial F_{k}}{\partial p}-\frac{\partial F_{i}}{\partial p} \frac{\partial F_{k}}{\partial q}$, and $H=F_{1}$. Geometrically $F_{i}$ and $F_{k}$ are orthogonal.

The effect is that the trajectories of the system must lie on the $n$-dimensional phase surface $F_{i}(p, q)=k_{i}$ and the involution requirement restricts the surface to be an $n$-dimensional toroid. The $\omega(I)=\frac{\partial H_{0}(I)}{\partial I}$ are interpreted as an angular velocity vector on the $n$-torus. The trajectories are $n$ frequency quasi-periodic if there is no vector of integers $m=\left(m_{1}, \ldots, m_{n}\right)$ such that $m \cdot \omega(I)=0$. Alternatively for the case of $n$ frequency periodic motion such a vector exists and $m \cdot \omega(I)=0$. A point $I$ in the base $B$ is called a resonant point if the vector $\omega(I)$ is a resonance vector.

\subsubsection{Perturbed integrable systems}

The system (3.54), (3.55) is integrable as in the time scale $\frac{1}{\varepsilon}$ the equations in $\phi$ are trivially linear. This only occurs in special cases. But if a system is nearly integrable in that $H(p, q)$ where $p=p_{1} \ldots p_{n}, q=q_{1} \ldots q_{n}$, contains a small parameter $\varepsilon$ and introduction of action/angle coordinates produces the system:

$$
\begin{aligned}
\dot{I} & =\varepsilon f(I, \phi), \\
\dot{\phi} & =\omega(I)+\varepsilon g(I, \phi),
\end{aligned}
$$


so that if $\varepsilon=0$ this system is integrable and if $0<\varepsilon \ll 1$ then the system is called nearly integrable and can be analysed using perturbation techniques.

Clearly a system remains Hamiltonian if the perturbation is Hamiltonian. The trajectories must follow constant energy manifolds as determined by the Hamiltonian. If the system is integrable then the $n$ constants of motion $F_{i}$ imply that the trajectories must lie on an $n$-dimensional torus. The structure of the solution is stable where they are quasi-periodic, but perturbation around the resonances leads to larger changes in the solution structure.

Although most Hamiltonian systems are non-integrable they are often studied as perturbations of integrable systems. The effect of a nonintegrable perturbation is that the constants of motion $F_{i}$ no longer hold. Supposedly the $n$-dimensional torus structure would be destroyed but Kolmogorov-Arnold-Moser theory shows that the torus structure is maintained for small values of $\varepsilon$ with complicated dynamics between the tori surfaces. Where there are homoclinic loops between the tori, these can bifurcate into a chaotic homoclinic entanglement. The constant energy surfaces do not all respond uniformly to nonintegrable perturbation. The resonant tori, characterised by $m \cdot \omega(I)=0$ in action/angle coordinates are destroyed by perturbation, being replaced by varied dynamics, while the non-resonant tori remain. But the system remains Hamiltonian, there are no attractors.

\subsubsection{Dynamics near resonant trajectories}

The solution near resonant manifolds can be studied by introducing local variables. Assume coordinates $\left(I_{1}, \phi_{1}\right)$, such that the resonant manifold lies in the $I_{1}=0$ plane. Then introduce $I_{1}=\delta(\varepsilon) \xi$. The layer near $I_{1}=$ 0 has the local variable $I_{1}=\delta(\varepsilon) \xi$, where $\delta(\varepsilon)=o(1)$ as $\varepsilon \rightarrow 0$ and is determined through balancing the perturbation terms in the dynamical 
equations (so that each equation is similarly perturbed). $\phi_{1}$ is replaced by $\psi=m \cdot\left(\phi_{1} \ldots \phi_{n}\right)$, so at resonance $\dot{\psi}=0, \psi=$ constant, resonance is an equilibrium point for $\psi$, and $\dot{\psi}=\delta(\varepsilon) \xi \cdot m \cdot \frac{\partial \omega\left(0, I_{2} \ldots I_{m}\right)}{\partial I_{1}}$ to first order. The dynamical equations are now in $\delta(\varepsilon) \dot{\xi}, \dot{I}_{2} \ldots \dot{I_{m}}, \dot{\psi}, \dot{\phi}_{2} \ldots \dot{\phi}_{n}$. It is found that $\delta(\varepsilon)=\sqrt{\varepsilon}$, and that $\psi$ obeys a second order pendulum like system (with potential force $\left.\frac{\partial V}{\partial \psi}=\varepsilon f_{1}\left(\left(0, I_{2} \ldots I_{m}\right),\left(\psi, \phi_{2} \ldots \phi_{n}\right)\right) \cdot m \cdot \frac{\partial \omega\left(0, I_{2} \ldots I_{m}\right)}{\partial I_{1}}\right)$, that is a two dimensional dynamical system in a time scale of $\frac{1}{\sqrt{\varepsilon}}$. It can be seen that this system is conservative as $\operatorname{trace}(D[\dot{\xi}, \dot{\psi}])=0$. This is in spite of any dissipation in the original system. However as the time scale is only of order $\frac{1}{\sqrt{\varepsilon}}$ a second order approximation is warranted. This could lead to center points becoming attractors or repellers.

\subsection{Melnikov's method}

\subsubsection{Introduction}

The formulation of a problem in the Lagrange form $\dot{x}=\varepsilon f(x, t, \varepsilon)$ is more difficult for strongly nonlinear systems of the form $\dot{x}=f(x)+\varepsilon g(x, t)$. $f(x)$ is assumed Hamiltonian and integrable, and $\varepsilon g(x, t)$ is a small perturbation, $T$-periodic in $t$, which need not be Hamiltonian. Melnikov's method gives information on global behaviours of a system in particular the homoclinic bifurcation of the homoclinic loop to a saddle point of a planar system. The global solution of the unperturbed system is used in the computation of the perturbed solution.

\subsubsection{Geometry of a homoclinic loop}

Let $f(x)$ have a homoclinic orbit $q^{0}(t)$, with a hyperbolic saddle point $p_{0}$. $\Sigma^{t_{0}}$ is a cross section of the non-autonomous flow (where $\dot{\theta}=1$ ), at time $t_{0}$. The Poincaré map $P_{\varepsilon}^{t_{0}}$ maps $\Sigma^{t_{0}} \rightarrow \Sigma^{t_{0}}$. The stable and unstable manifolds 
of the perturbed system are perturbations of $q^{0}(t)$ so that:

$$
\begin{array}{r}
q_{\varepsilon}^{s, u}\left(t, t_{0}\right)=q^{0}\left(t-t_{0}\right)+\varepsilon q_{1}^{s, u}\left(t, t_{0}\right)+O\left(\varepsilon^{2}\right) \text { for } W_{s}: t \epsilon\left[t_{0}, \infty\right), \\
W_{u}: t \epsilon\left(-\infty, t_{0}\right] .
\end{array}
$$

Therefore $q_{1}^{s, u}$ is found from the first variational equation:

$$
\dot{q}_{1}^{s, u}\left(t, t_{0}\right)=D f\left(q^{0}\left(t-t_{0}\right)\right) q_{1}^{s, u}\left(t, t_{0}\right)+g\left(q^{0}\left(t-t_{0}\right), t\right) .
$$

This is obtained from substituting the perturbation expansion of $q_{\varepsilon}^{s, u}\left(t, t_{0}\right)$ into the system and solving for the terms in $\varepsilon$ for $q_{1}^{s, u}$, where $q^{0}(t)$ is the solution of the zero order equation in the equation hierarchy.

Let $d\left(t_{0}\right)=q_{\varepsilon}^{u}\left(t_{0}\right)-q_{\varepsilon}^{s}\left(t_{0}\right)$, where $q_{\varepsilon}^{s, u}\left(t_{0}\right)=q_{\varepsilon}^{s, u}\left(t_{0}, t_{0}\right)$. This is determined from the intersection of the normal through $q^{0}(0)$, a point on the unperturbed homoclinic loop, with the perturbed stable and unstable manifolds at time $t_{0}$. So

$$
d\left(t_{0}\right)=\varepsilon \frac{f\left(q^{0}(0)\right) \wedge\left(q_{1}^{u}\left(t_{0}\right)-q_{1}^{s}\left(t_{0}\right)\right)}{\mid f\left(q^{0}(0) \mid\right.}+O\left(\varepsilon^{2}\right),
$$

where $a \wedge b$ is the wedge product $=a_{1} b_{2}-a_{2} b_{1}$, as $\left(b_{1}, b_{2}\right)^{\perp}=\left(-b_{2}, b_{1}\right)$. This is the projection of $q_{\varepsilon}^{u}\left(t_{0}\right)-q_{\varepsilon}^{s}\left(t_{0}\right)$ on the normal $f^{\perp}$, which is the closest distance of the unstable and unstable manifolds to the point $q^{0}(0) \cdot q^{0}(0)$ remains the same point on the homoclinic loop $\forall t_{0}$ of the autonomous flow whenever $t_{0}$ is defined, as $q^{0}(0)$ is defined in the unperturbed system at time $t=0$, whereas $q_{1}^{s, u}\left(t_{0}\right)$ varies with $t_{0}$, i.e. it is parameterised by $t_{0}$, as $d\left(t_{0}\right)$ changes with $t_{0}$ at the same point on the unperturbed trajectory.

The Melnikov function is then defined as:

$$
M\left(t_{0}\right)=\int_{-\infty}^{\infty} f\left(q^{0}\left(t-t_{0}\right)\right) \wedge g\left(q^{0}\left(t-t_{0}\right), t\right) d t,
$$

$d\left(t_{0}\right)=\frac{\varepsilon M\left(t_{0}\right)}{\mid f\left(q^{0}(0) \mid\right.}+O\left(\varepsilon^{2}\right)$ as from,

$$
\begin{aligned}
\Delta\left(t, t_{0}\right) & =f\left(q^{0}\left(t-t_{0}\right)\right) \wedge\left(q_{1}^{u}\left(t, t_{0}\right)-q_{1}^{s}\left(t, t_{0}\right)\right), \\
& =\Delta^{u}\left(t, t_{0}\right)-\Delta^{s}\left(t, t_{0}\right),
\end{aligned}
$$


the time varying distance function (wrt $t_{0}$ ), and

$$
\dot{\Delta}^{u, s}=\operatorname{trace} D f\left(q_{0}\left(t-t_{0}\right)\right) \Delta^{u, s}+f\left(q^{0}\left(t-t_{0}\right)\right) \wedge g\left(q^{0}\left(t-t_{0}, t\right)\right),
$$

from the variational equation. $\operatorname{trace}(D f)=0$ as $f$ is Hamiltonian, and integrating $\dot{\Delta}^{u}$ over $\left(-\infty, t_{0}\right]$ and $\dot{\Delta}^{s}$ over $\left[t_{0}, \infty\right)$ gives $\Delta^{u}\left(t_{0}, t_{0}\right)+\Delta^{s}\left(t_{0}, t_{0}\right)=$ $M\left(t_{0}\right)=d\left(t_{0}\right) \mid f\left(q^{0}(0) \mid+O\left(\varepsilon^{2}\right)\right.$, as $\Delta^{u}\left(-\infty, t_{0}\right)-\Delta^{s}\left(\infty, t_{0}\right)=0$ because the manifolds intersect at the saddle point $p_{0}^{\varepsilon}$.

\subsubsection{Bifurcations of a homoclinic loop}

What are the possible dynamics of the bifurcation of a homoclinic loop? The unstable and stable manifolds might simply cross over and the direction of the flow reverse. A limit cycle might appear or vanish in the interior of the loop depending on the direction of the parameter adjustment. If $M\left(t_{0}\right)$ has simple zeros and is independent of $\varepsilon$ then for $\varepsilon>0$, sufficiently small the stable and unstable manifolds intersect transversely. If $M\left(t_{0}\right) \neq 0$ for any $t_{0}$ then the manifolds do not intersect anywhere. The implication of this is that the traverse homoclinic orbits show the existence of a Smale horseshoe and therefore chaotic dynamics.

Similarly a Melnikov function can used to analyse the stability of a limit cycle inside a homoclinic loop. Let $q^{\alpha}\left(t-t_{0}\right)$ be a periodic orbit of the unperturbed system with period $T_{\alpha}$, then there exists an orbit $q_{\varepsilon}^{\alpha}\left(t, t_{0}\right)$ not necessarily periodic which can be expressed as:

$$
q_{\varepsilon}^{\alpha}\left(t, t_{0}\right)=q^{\alpha}\left(t-t_{0}\right)+\varepsilon q_{1}^{\alpha}\left(t, t_{0}\right)+O\left(\varepsilon^{2}\right),
$$

uniformly in $t \epsilon\left[t_{0}, t_{0}+T_{\alpha}\right]$, for $\varepsilon$ sufficiently small. The subharmonic Melnikov function is defined as:

$$
M^{\frac{m}{n}}\left(t_{0}\right)=\int_{0}^{m T} f\left(q^{\alpha}(t)\right) \wedge g\left(q^{\alpha}(t), t+t_{0}\right) d t,
$$

where $q^{\alpha}\left(t-t_{0}\right)$ is a periodic orbit of period $T_{\alpha}=\frac{m}{n} T$, and $T$ is the period of the perturbation. If $M^{\frac{m}{n}}\left(t_{0}\right)$ has simple zeroes, and is independent of $\varepsilon$, 
and $\frac{\partial T_{\alpha}}{\partial h_{\alpha}} \neq 0$, where $h_{\alpha}=H\left(q^{\alpha}(t)\right)$, then there exists a subharmonic orbit of period $m T$. The subharmonic Melnikov function measures the movement of the perturbed trajectory on the Poincare plot for $m$ revolutions of the torus $\equiv n$ cycles of the periodic orbit. So if $t_{0}$ is at a fixed point then the Melnikov function is zero. 


\section{Chapter 4}

\section{Mathematical Modelling of the Cardiovascular System}

\subsection{Introduction}

The motivation for the derivation of a more complicated map for heart rate interval is that the existing map [26] used to demonstrate Arnold tongues in coupling between the respiratory and cardiovascular systems has limited dynamics and in particular can not exhibit chaos. A more elaborate map is thought to be helpful to exhibit a wider range of possible behaviour for the heart interval in order for the dynamics of coupling between the respiratory and cardiovascular systems to be explored more fully. The approach is to model the heart interval with diffential equations and to derive a map from these. Then the map is generalised and its parameters associated with significant parameters of heart physiology. Some physiology of the human respiratory and cardiovascular systems is described in Appendix A. 


\subsection{Background}

Recent models of the cardio-vascular system are based on the Windkessel model (DeBoer et al.[27]) of an arterial compartment characterised by a compliance and a fluid outflow controlled by capillary resistance. J. T. Ottesen [28] has developed a non-pulsatile model of the cardio-vascular system to study the effects in the time delay of the parasympathetic and sympathetic control. The left ventricle is treated as a continuous source of fluid quantified by a heart rate $H$ and a stroke volume $V_{\text {str }}$. There are two compartments characterised by a compliance with dimensions of volume/pressure, $c_{a}, c_{v}$ modeling the aorta and the veins going into the right side of the heart. Connecting the two compartments is the capillary system modelled by a resistance $R$. As the right ventricle removes the fluid it is effectively a sink at a pressure potential of zero. The veins supply fluid to the right ventricle via the right atrium and the flow into the right atrium is modelled by a resistance $r$. The state variables are the mean fluid pressure in the aorta and the veins, $P_{a}(t), P_{v}(t)$. This leads to two differential equations for the uncontrolled non-pulsatile cardiovascular system:

$$
\left(\begin{array}{c}
\dot{P}_{a}(t) \\
\dot{P}_{v}(t)
\end{array}\right)=\left(\begin{array}{cc}
-\frac{1}{c_{a} R} & \frac{1}{c_{a} R} \\
\frac{1}{c_{v} R} & -\frac{1}{c_{v}}\left(\frac{1}{r}+\frac{1}{R}\right)
\end{array}\right)\left(\begin{array}{c}
P_{a}(t) \\
P_{v}(t)
\end{array}\right)+\frac{1}{c_{a}} H V_{\text {str }}\left(\begin{array}{l}
1 \\
0
\end{array}\right) .
$$

The effect of the baroreflex control is added to this system by introducing $H$ the heart rate, as an additional state variable instead of a constant, which is controlled by a function $f$ of $P_{a}(t)$. As the parasympathetic delay is much less than the sympathetic delay (about 10x), the parasympathetic delay is neglected and $f$ is a function of $P_{a}(t)$, and $P_{a}(t-\tau) . f$ is a composition of $h$, a 2 dimensional function giving the effect of the parasympathetic tone and the sympathetic tone on the heart rate, and $g$, the $S$ curve response of the baroreceptor which gives the parasympathetic tone and the sympathetic tone as a parameterised function of $P_{a}(t)$, and $P_{a}(t-\tau)$ respectively. 
The third equation of the model for the heart rate is then

$$
\dot{H(t)}=f\left(P_{a}(t), P_{a}(t-\tau)\right)
$$

Ottesen analyses the stability of this system by linearising the model around the equilibrium point and studies the characteristic equation. The effect of the time delay is to introduce a term $Q(\lambda) e^{-\lambda \tau}$ in the usual characteristic polynomial equation of a linear system. A 2 parameter space is divided into regions of one positive root, two positive roots, and no positive roots. Ottesen finds he is able to explain the phenomenon of $10 \mathrm{~Hz}$ Mayer waves of blood pressure by changes in the sympathetic time delay with a sensitivity to $R$, and that the model responds realistically to step changes in $R$ caused by physical exercise which is verified by experimental data. Ottesen also considers the effect of the simplification $r=0$, as well as the effect of a nonconstant stroke volume.

Fowler and McGuiness [29] extend this approach simplifying the model to a single delay/ recruitment equation. This is linearized to analyse the stability, giving a transcendental equation $\sigma=-B-G \mathrm{e}^{-\sigma}$. The $B, G$ parameter plane contains regions of stability, damped oscillation and, unstable oscillation separated by a Hopf bifurcation curve.

To study the phenomenon of cardiovascular synchronisation a pulsatile model is needed as it is the dynamics of the heart phase that lead to synchronisation. Thus rather than a heart rate equation as for the nonpulsatile model, a heart phase dynamical equation is introduced. McGuinness et al. [26] study cardiovascular synchronisation abstracting the cardiovascular system as two relaxation oscillators. Respiratory coupling is via modulation of the integration rate. During the respiration cycle the heart rate is slowed during the first half of the cycle and increased during the second half of the cycle. A 1D map of heart interval in terms of respiration phase is given. The deviation of the cumulative respiration phase from a constant rate, at its maximum, is given by the coupling parameter $\epsilon$ in the middle of the respiration cycle. Cardio-vascular coupling is a pulse 
coupling whereby the respiration cycle is shortened if a heart beat occurs close enough to the end of the respiration cycle. The parameter $A$ is the amplitude of the phase pulse and if the addition of this pulse to the current phase crosses the 1 threshold a new respiration cycle is started.

There have been several studies in the past of coupled relaxation oscillators, either 2 or $N$ oscillators. These use various pulse coupling schemes. A. A. Brailove [1] has each discharge of the relaxation oscillator providing a pulse to the coupled oscillator(s) which is integrated. Chia-Chu Chen [30] introduces a threshold effect where coupled pulses in the initial part of the integration have no effect. This is exactly analogous to the phase effectiveness curve of the heart oscillator. The scheme of McGuinness et al. can be seen to exhibit both extremes of strength in the coupling as well as representing a model of the cardiovascular system. It can be seen that coupling of the respiration to the heart is via a $O(\varepsilon)$ strength perturbation of a constant rate $f_{\mathrm{r}}$ relaxation oscillator representing the effect of Respiratory Sinus Arrhythmia (RSA, see Appendix A), on the oscillator threshold. Graphically the triangular deviation of the oscillator map intersects the $\theta_{i+1}=\theta_{i}$ line in a tangent bifurcation to generate the locking zones, depending on the perturbation $\varepsilon$ and the nominal period of the relaxation oscillator $T=\frac{1}{f_{\mathrm{r}}}$. Similarly a pulse coupled scheme results in the phase difference of the coupled oscillators drifting until the stable fixed point created by the pulse coupling is reached. The locking time depends on the width of the pulse and the width of the locking zone on the height of the pulse.

H. Seidel and H. Herzel [31] model the cardiovascular system using a pulsatile model. This is a system of differential equations based on the Windkessel model as for Ottesen. The pulsatility is expressed by dividing the heart cycle into the systolic part where the heart is contracting and the diastolic part where the fluid in the aorta is squeezed into the vascular system. Whereas the fluid pressure in the diastolic part is expressed using the simple Windkessel model $\dot{p}=-\frac{p}{\tau_{v}(t)}$, the pressure in the systolic part of 
the cycle is a pressure pulse driving function $p=d_{i-1}+S_{i} \frac{t-t_{i}}{\tau_{\mathrm{sys}}} \exp \left\{1-\frac{t-t_{i}}{\tau_{\mathrm{sys}}}\right\}$. The Seidel and Herzel model is elaborated in several directions. The Windkessel time constant is time varying. This reponds to baro-receptor sympathetic activity. Additionally the metabolism of the sympathetic transmitter noneprephrine is expressed in a first order delay differential equation for the vascular concentration. The delay of $1.65 \mathrm{~s}$ is due to the transmission of nervous impulses and the transport of the neurotransmitter. The heart rate is controlled by para-sympathetic and sympathetic loops where the Ottesen model uses only the sympathetic loop. These have differing delays, $.3 \mathrm{~s}$ and $1.65 \mathrm{~s}$.

There is no equation for the para-sympathetic neuro-transmitter as this metabolises quite quickly but there is an equation for the cardiac concentration of the sympathetic neuro-transmitter. This variable affects both the sympathetic influence on the sino-atrial node and the contractility of the heart $S_{i}$ and therefore the systolic pressure. Due to the particular chemistry of the parasympathetic transmitter there is a phase effectiveness curve where the effect of the parasympathetic activity is dependent on the phase of the sino-atrial node.

Cross coupling between the parasympathetic and sympathetic loops is expressed by their product being in the denominator of the time constant of the sino-atrial node. RSA is modelled by a sine half wave term, at the respiration frequency, in the equations for para-sympathetic and sympathetic activity. This models RSA as centrally coupled in the nervous system rather than mechanically coupled through the chest cavity. There is no cardiovascular coupling (CVC) in this model.

Seidel and Herzel numerically solve the system firstly to study the effect of changes in parameters. They observe a baro-receptor loop response peak of .1 Hz corresponding to damped oscillations which is some times a $10 \mathrm{sec}$ sustained rhythm corresponding to Mayer waves. The effect of RSA is observed as a variation in the heart interval at the respiration frequency.

Variations in the sympathetic time delay are observed to cause oscilla- 
tions in the heart interval corresponding to a Hopf bifurcation. Variations in the baro-receptor coupling, (baro-receptor gain) lead to limit cycles of the heart interval denoted as alternans - an arrhythmia of alternating heart periods, and an oscillation of the baro-reflex control loop, the superposition of which with the cardiac pacemaker constitutes a torus. As gain increases there is entrainment between the baro-receptor loop oscillation and the heart oscillator and finally chaos.

\subsection{Studying Synchronisation in the Cardio- vascular System}

The current study seeks to investigate the synchronisation in the cardiovascular system. As this is a function of the phase of the respiration and the cardiac oscillators a pulsatile model is needed.

The differential equations of Seidel and Herzel [31] are followed. This model is more fully explained in [32]. Initially a model of only the parasympathetic loop was studied as the synchronisation of the heart is seen to act through the phase effectiveness curve $F(\phi)$ of the sino-atrial node which is a function of the parasympathetic neurotransmitter chemistry. Subsequently the sympathetic loop was added to study the effect of the responsiveness of the heart oscillator to perturbation and the effect on synchronisation as there is a cross product term between the parasympathetic and sympathetic influences and the gain of the sympathetic loop is about $1.5 \mathrm{x}$ the parasympathetic loop before taking the phase effectiveness curve into account, so this should be the relative effect on synchronisation. As the phase effectiveness curve attenuates the effect of the nervous influence by on average $.05 \mathrm{x}$ it is the sympathetic loop that has the greatest effect on the response of the heart oscillator.

The systolic pressure is determined by the contractility of the heart which is related to the volume of fluid entering the heart and sympathetic 
nervous influence. The systolic pressure of the fluid built up at each stroke is modelled as proportional to the previous heart interval time. But this is an approximation as the volume retained in the peripheral system varies as the resistance to flow varies. This also assumes that the pulmonary system plays no dynamical role in the fluid flow, but as there is at least one heart interval delay for the effect of the current heart stroke to affect the returning fluid perhaps a dependence on the second to last heart interval time would be more realistic. Seidel and Herzel include in their model a system of equations for the contractility of the heart including sympathetic transmitter concentration in the heart. The systolic pressure at each stroke is a proportion of the previous interval time and the contractility of the heart added together.

The Windkessel time constant, a product of the resistance to flow and the compliance of the aorta is also modelled as a function of peripheral sympathetic transmitter concentration. The characteristic $S$ shaped function of the baroreceptor response is included as the transfer function of the parasympathetic activity to the parasympathetic influence as is the time delay and similarly for the sympathetic activity. The RSA is added centrally to the parasympathetic and sympathetic activity, rather than being considered as a mechanical effect of the chest cavity and added to baroreceptor activity. The following equations were used,

Baroreceptor activity $v_{b}$ :

$$
v_{b}=k_{1}\left(p-p^{0}\right)+k_{2} \frac{\mathrm{d} p}{\mathrm{~d} t} .
$$

Possibly $k_{2}$ is small and its effect must be limited by the saturation of the baroreceptor at the systolic pressure. $p^{0}$ is a parameter of the dynamic range of the baroreceptor.

Parasympathetic activity $v_{p}$ :

$$
v_{p}=\max \left(0, v_{p}^{(0)}+k_{p}^{b} v_{b}+k_{p}^{r}\left|\sin \left(\pi f_{r} t+\Delta \phi_{p}^{r}\right)\right|\right) .
$$

Sympathetic activity $v_{s}$ :

$$
v_{s}=\max \left(0, v_{s}^{(0)}-k_{s}^{b} v_{b}+k_{s}^{r}\left|\sin \left(\pi f_{r} t+\Delta \phi_{s}^{r}\right)\right|\right),
$$


where $\Delta \phi_{p, s}^{r}$ are initial conditions for the respiratory influence on the nervous activity, usually set equal to 0 .

Sinus node phase $\varphi$ :

$$
\frac{\mathrm{d} \varphi}{\mathrm{d} t}=\frac{1}{\boldsymbol{T}^{0}} f_{s} f_{p},
$$

$\boldsymbol{T}^{0}$ is the heart interval length generated by the sino-atrial node. $f_{s}$ is the sympathetic influence on the sino-atrial node.

Parasympathetic influence on the phase velocity of the sinus node $f_{p}$ :

$$
f_{p}=1-k_{\varphi}^{p}\left(v_{p}\left(t-\theta_{p}\right)+\left(\hat{v}_{p}-v_{p}\left(t-\theta_{p}\right)\right) \frac{v_{p}\left(t-\theta_{p}\right)^{n_{p}}}{\hat{v}_{p}^{n_{p}}+v_{p}\left(t-\theta_{p}\right)^{n_{p}}}\right) F(\varphi),
$$

$\theta_{p}$ is the time delay of the parasympathetic loop, about $.5 \operatorname{secs}, F(\varphi)$ is the phase effectiveness curve.

Cardiac concentration of the sympathetic transmitter $c_{c \mathrm{Na}}$ :

$$
\frac{\mathrm{d} c_{c \mathrm{Na}}}{\mathrm{d} t}=-\frac{c_{c \mathrm{Na}}}{\tau_{c \mathrm{Na}}}+k_{c \mathrm{Na}}^{s} v_{s}\left(t-\theta_{c \mathrm{Na}}\right),
$$

$\theta_{\mathrm{cNa}}$ is the transport delay of the sympathetic transmitter epinehrine, and $\tau_{\mathrm{cNa}}$ is the time constant for the metabolism of the transmitter.

Sympathetic influence on the phase velocity of the sinus node $f_{s}$ :

$$
f_{s}=1+k_{\varphi}^{c \mathrm{Na}}\left(c_{c \mathrm{Na}}+\left(\hat{c}_{c \mathrm{Na}}-c_{c \mathrm{Na}}\right) \frac{c_{c \mathrm{Na}}^{n_{c \mathrm{Na}}}}{\hat{c}_{c \mathrm{Na}}^{n_{c \mathrm{Na}}}+c_{c \mathrm{Na}}^{n_{c \mathrm{Na}}}}\right) .
$$

Phase-effectiveness curve F:

$$
F(\varphi)=\varphi^{1.3}(\varphi-0.45) \frac{(1-\varphi)^{3}}{(1-0.8)^{3}+(1-\varphi)^{3}} .
$$

Instantaneous fluid pressure $p$ :

$$
\frac{\mathrm{d} p}{\mathrm{~d} t}=\frac{-p}{\tau_{v}(t)}+S_{n} \cdot t \cdot \exp \left(-\frac{(t-.04)^{2}}{.00125}\right) k_{I},
$$

$S_{n}$ is the contractility. $k_{I}$ is a constant which normalises the area under the pulse function and includes the compliance of the aorta so that the change in pressure depends on the contractility. $\tau_{v}(t)$ is the Windkessel time constant which is time varying and depends on the peripheral sympathetic 
transmitter concentration. This equation is an adaption of the Seidel and Herzel equation so that the system is a continuous rather than piecewise continuous as in the implementation of Seidel and Herzel where the systolic and diastolic intervals are considered separately.

Windkessel time constant $\tau_{v}$ :

$$
\tau_{v}=\tau_{v}^{(0)}-\hat{\tau_{v}}\left(c_{v \mathrm{Na}}+\left(\hat{c}_{v \mathrm{Na}}-c_{v \mathrm{Na}}\right) \frac{c_{v \mathrm{Na}}^{n_{v N a}}}{\hat{c}_{v \mathrm{Na}}^{n_{v N}}+c_{v \mathrm{Na}}^{n_{v \mathrm{Na}}}}\right) .
$$

The Windkessel time constant including the fixed compliance of the artery and a varying peripheral resistance is a Hills function of peripheral sympathetic transmitter concentration with several parameters. The $\hat{c}_{v \mathrm{Na}}$ value is the limiting value of the function and the exponent controls the curvature. The function is linear for low values of the argument.

Peripheral sympathetic transmitter concentration $c_{v \mathrm{Na}}$ :

$$
\frac{\mathrm{d} c_{v \mathrm{Na}}}{\mathrm{d} t}=-\frac{c_{v \mathrm{Na}}}{\tau_{v \mathrm{Na}}}+k_{c_{v \mathrm{Na}}}^{s} v_{s}\left(t-\theta_{v \mathrm{Na}}\right),
$$

where $\tau_{v \mathrm{Na}}$ is the metabolic time constant of the sympathetic transmitter, $\theta_{v \mathrm{Na}}$ is the delay of the sympathetic nervous influence, including electrical delay in the nervous system and delay time for the passage of the epinehrine across the synapse to the peripheral system.

Cardiac contractility without saturation $S_{n}^{\prime}$ :

$$
S_{n}^{\prime}=S^{(0)}+k_{S}^{c} c_{c \mathrm{Na}}+k_{S}^{t} t_{n-1}
$$

The contractility is based on a constant value with additive terms proportional to the cardiac sympathetic transmitter concentration and the length of the previous heart interval time.

Cardiac contractility with saturation $S_{n}$ :

$$
S_{n}=S_{n}^{\prime}+\left(\hat{S}-S_{n}^{\prime}\right) \frac{S_{n}^{\prime n_{s}}}{S_{n}^{\prime n_{s}}+\hat{S}^{n_{s}}} .
$$

The contractility is limited with a Hills function. 


$\begin{array}{llllll}\mathrm{p}^{(0)} & 50.0 \mathrm{~mm} \mathrm{Hg} & k_{S}^{t} & 10 \mathrm{~mm} \mathrm{Hg} \mathrm{s}^{-1} & S^{(0)} & 25 \mathrm{~mm} \mathrm{Hg} \\ k_{1} & .02 \mathrm{~mm} \mathrm{Hg}^{-1} & \hat{S} & 70.0 \mathrm{~mm} \mathrm{Hg} & k_{S}^{c} & 40 \mathrm{~mm} \mathrm{Hg} \\ k_{2} & .00125 \mathrm{~s} \mathrm{~mm} \mathrm{Hg}^{-1} & k_{c \mathrm{Na}}^{s} & 1.2 & \theta_{p} & 0.5 \mathrm{~s} \\ v_{p}^{0} & 0.0 & \theta_{c \mathrm{Na}} & 1.65 \mathrm{~s} & \tau_{c \mathrm{Na}} & 2.0 \mathrm{~s} \\ k_{p}^{b} & .3 & k_{\varphi}^{c \mathrm{Na}} & 1.6 & n_{s} & 2.5 \\ k_{p}^{r} & .1 & \hat{c}_{c \mathrm{Na}} & 2.0 & n_{p} & 2.0 \\ \Delta \phi_{p}^{r} & 0 & \Delta \phi_{s}^{r} & 0 & \theta_{v \mathrm{Na}} & 1.65 \mathrm{~s} \\ f_{r} & .2 \mathrm{~s}^{-1} & k_{I} & 346.94 & \hat{v}_{p} & 2.5 \\ v_{s}^{(0)} & .8 & \tau_{v}^{(0)} & 2.2 \mathrm{~s} & k_{c_{v N a}}^{s} & 1.2 \\ k_{s}^{b} & .7 & \hat{\tau}_{v} & 1.2 \mathrm{~s} & k_{\varphi}^{p} & 5.8 \\ k_{s}^{r} & .1 & \hat{c}_{v \mathrm{Na}} & 10.0 & \tau_{v \mathrm{Na}} & 2.0 \mathrm{~s} \\ \boldsymbol{T}^{0} & 1.1 \mathrm{~s} & n_{v \mathrm{Na}} & 1.5 & n_{c \mathrm{Na}} & 2.0\end{array}$

Table 4.1: Table of nominal parameter values

\subsubsection{Numerical solution of system}

The heart oscillator was modelled using the DDE23 differential delay system solver in MatLab. The system of equations (4.1)-(4.13) has 5 state variables because as well as the heart phase $\varphi(4.4)$, fluid pressure $p(4.9)$, equations, and cardiac (4.6) and peripheral (4.11) concentrations of sympathetic transmitters $c_{c \mathrm{Na}}, c_{v \mathrm{Na}}$, the previous heart beat time interval $t_{n-1}(4.12)$ is used to determine the volume of blood pumped in the heart beat at the start of the heart oscillation. So the 4 state variable system used is nonautonomous as it is driven by this quantity. This represents an implicit integration of the inverse phase function to find the heart interval.

The DDE23 solver can solve a non-autonomous system of equations with a finite number of delays. Therefore it is capable of solving the heart equations numerically with both the parasympathetic and sympathetic loops. Initially only the parasympathetic loop was studied. Subsequently the sympathetic loop and peripheral resistance was added to study the effect. 
The DDE23 solver requires as input a history function which specifies the history of the system dependent variables up to the longest delay. For the system studied only the pressure variable is delayed so only this history function is required. As the exact history is unknown it is approximated initially using nominal values and the Windkessel model for aortic pressure. The $t_{-1}$ is an initial condition of the system, as is $d_{-1}$ the diastolic pressure at the end of the heart interval (identical to $p(t)$ at $\phi=1$ ). These are set to appropriate values when the model is started. The $t_{n-1}$ is then iterated for a number of heart cycles to remove the transient effect of the initial inaccuracy in the history function.

It was found that, as the step size is adaptive, the pulsatile nature of the system caused problems with the DDE23 solver. That is as the pressure pulse of the heart beat was continuous rather than discontinuous the solver did not track the rapid change in the pressure state variable very well. Therefore the system was solved one heart interval at a time. This meant that an approximate history function was inadequate, as the solver would require an accurate history prior to the initial condition, so the output of the solver for each interval needed to be stored in an array for a time, up to the maximum delay in the system. The history function supplied to the solver looked up values in the history array, when the solver needed state values beyond the length of the current interval calculation. Otherwise the history function uses the state values saved by the DDE23 solver.

The DDE23 solver has a number of options which increase its usefulness. The usual relative and absolute tolerance can be set. A particular option which is used in the solution of the heart interval is the 'Events' option. An event function handle is supplied in the options field of the function call which monitors the steps of the solution and detects a zero crossing in either direction of a function of the solution state, and then can stop the integration. In the solution of the heart interval length this facility is used to detect the end of the heart phase cycle at $\phi=1$. The integration 
is then stopped at the exact heart interval length instead of having to intergrate over a fixed time interval longer than the heart interval length and then search the solution data for the end of the heart phase cycle. Another option is 'discontinuities' which restarts the integration at given discontinuities in the solution corresponding to initial conditions of a solution which uses the previous solution as the history function.

This is a similar approach to Seidel and Herzel [31] who use a 4th order Runge-Kutta algorithm (so the error is $O\left(h^{5}\right)$, where $h$ is the step size), with a fixed step size less than the smallest delay, to simplify programming, and a ring buffer for the solution history. The DDE23 uses a RungeKutta BS $(2,3)$ algorithm as described by Shampine et al. [33]. In this case therefore the integrator error is of order $O\left(h^{4}\right)$, whereas the error estimate for the stepsize control is of order $O\left(h^{3}\right)$.

After a fixed number of cycles to remove the grossest transient the rotation number of the heart oscillator with respect to the respiration oscillator is calculated until it has stabilised. This is taken to be relative to the rotation number to allow the variation of the rotation number with respiration frequency to be distinct. As the rotation number varies a little with the respiration phase this will take a number of respiration cycles. About 5000 heart intervals were calculated at each respiration frequency to find the rotation number as it took this length of time to average out the effect of the variation in the heart interval in a respiration cycle. While the nonlinearity of the phase effectiveness curve is necessary to difference the phases of the respiration and heart interval and generate a synchronizing force, the phases are also added generating a higher frequency component and the respiration fundamental component is also present causing significant jitter in the rotation number over a respiration cycle. Without synchronising the calculation to respiration cycle, which proved to be difficult because of the jitter, a large number of heart intervals was needed to find a precise rotation number. 


\subsubsection{Behaviour of heart system model}

The system seems quite elaborate for a study of oscillator coupling but it was found that the entire system is involved in the coupling dynamics so that all the equations of Seidel and Herzel are necessary for practical results. It is the peripheral resistance that has the greatest effect on fluid pressure and the Windkessel constant adjusts to maintain an approximately constant diastolic pressure via the sympathetic loop as a result of the high gain of this reflex. However RSA coupling occurs via the phase effectiveness curve of the parasympathetic loop altering the heart rate, which affects the fluid pressure, such that the peripheral control loop is counteracting the RSA. The fluid flow is related to the pressure via the compliance of the aorta. So the flow rate is proportional to the difference between the systolic and diastolic pressures or the contractility. Increasing contractility implies increased flow due to higher average pressure and increased heart rate also implies increased volume.

Seidel and Herzel model the heart stroke piecewise through adding an exponential pulse function onto the diastolic pressure of the previous heart interval during a time period called the systolic interval and then solve the Windkessel model during the diastolic interval. For a continuous model the differentiated step function is added to the Windkessel model for the systolic interval so that the differential equation is valid throughout the heart interval. The piecewise method allows a replica of the physiological situation, where a bump occurs at the systolic peak as fluid back pressure closes the heart valve, but neglects the effect of peripheral flow during the systolic interval perhaps affecting the heart interval time. The nonpiecewise method used equated the area under an $\mathrm{e}^{(x-a)^{2}}$ pulse function to the pressure increase of the heart stroke and added the pulse function to the Windkessel model (4.9).

The system of Seidel and Herzel includes a model of heart contractility and the system was found to be sensitive to these equations also. The heart contractility depends on the filling time via the myocardial fibres and the 
cardiac sympathetic transmitter concentration. It can be seen that where the contractility would be purely proportional to the filling time then this has the effect of degenerating the sympathetic loop gain as the fluid pressure would decrease with shorter heart intervals, whereas the effect of the sympathetic loop is to increase pressure through increasing heart rate. In this case of contractility proportional to filling time it was found that the bifurcation to oscillations with increased sympathetic time delay did not occur. Using a fixed constant for the contractility it was found that the bifurcations were sensitive to the value of the constant. For higher values of fixed contractility only damped oscillations were present and these became stable oscillations at a lower value. This seems to be an effect of nonlinearities in the $\mathrm{S}$ curves and the operating level of the system as the higher value constant increased the heart interval length and the average pressure level. The contractility was found to be essential to maintaining the system equilibrium point or pressure level. With the contractility as a constant to govern the pressure level, the equilibrium point was lost at about $80 \mathrm{mmHg}$ diastolic pressure. That is to say the pressure would decrease to zero and the system cease to function. The effect worsened with the contractility depending only on the previous interval length. The Seidel and Herzel equations model the contractility as a fixed term plus a term proportional to the previous time interval length and a term proportional to a saturating function of the transmitter concentration. As increasing transmitter concentration decreases the heart interval, this cancels the degenerating effect on the sympathetic loop gain, of the dependence of the contractility on the previous heart interval.

Figure (4.1) shows output of the model for typical parameters. The timescale of the graphs of parasympathetic and sympathetic activity are shifted by the respective delays wrt the pressure to indicate at what time they affect the phase progression although the graphs are still synchronised with the pressure graph. To validate the model some parameters were varied to compare the results with those of Seidel and Herzel. Fig- 

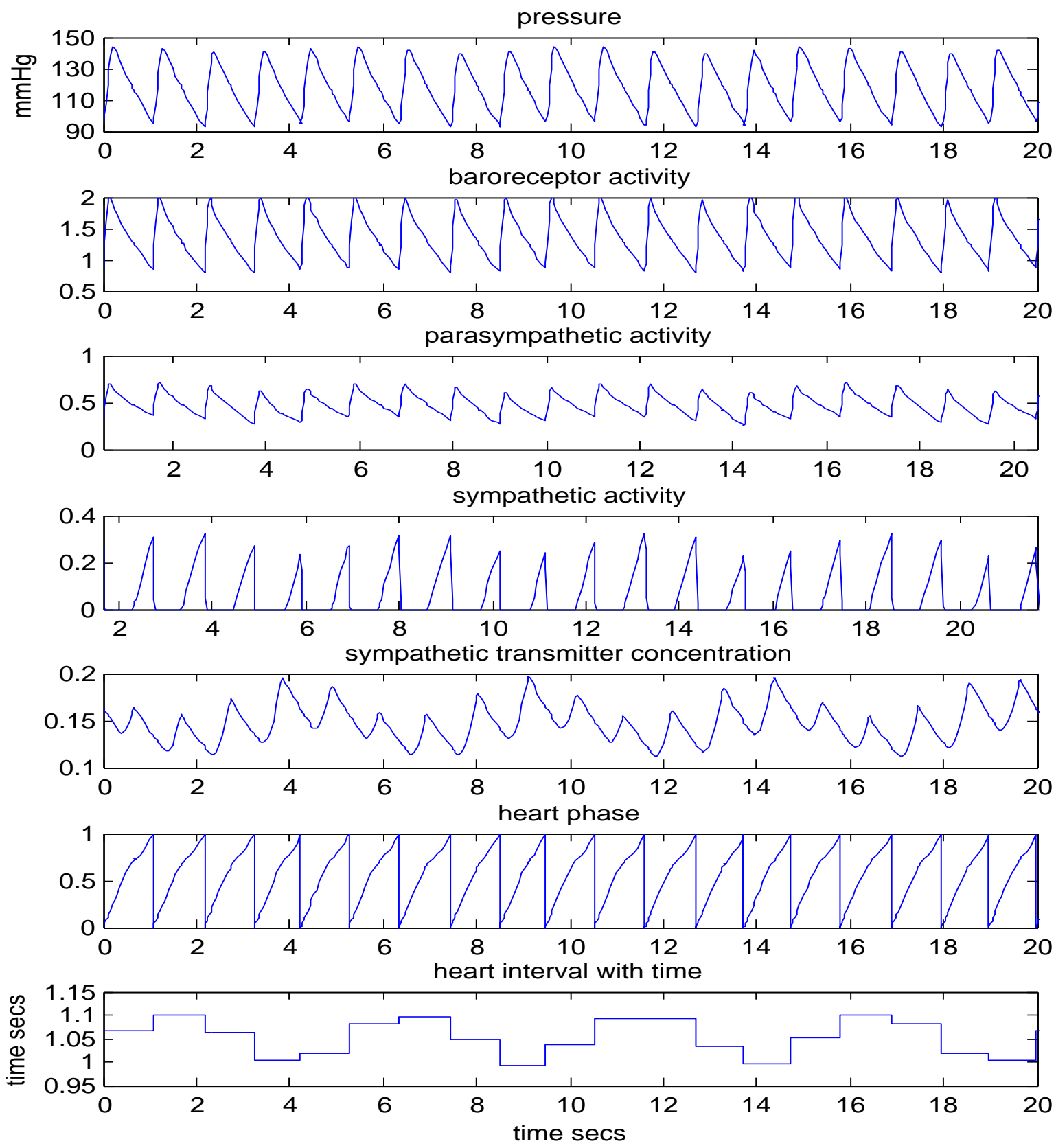

Figure 4.1: Output of the heart system model 
ures (4.2)-(4.3), show the effect of increasing the sympathetic loop delay from $t=1.65 \mathrm{~s}$ to $t=2.5 \mathrm{~s}$. The results are nearly identical to those of Seidel and Herzel. It was found that the model is very sensitive to the contractility (systolic pressure). A variation of +2.5 percent would quench the oscillation or -2.5 percent would double the amplitude. This is attributed to the baroreceptor S curves compressing the amplitude of the parasympathetic and sympathetic responses, reducing the loop gain and limiting the amplitude of the oscillation, and so the stability is sensitive to the average pressure level.

Another parameter tested was the parasympathetic loop gain. At higher levels of gain an alternans arrhythmia was found as per Seidel and Herzel, and loop oscillation which could be entrained. See figure (4.4). This entrainment employs the same phase reponsiveness curve mechanism as the RSA. The explanation for this effect depends on the delay times of the parasympathetic and sympathetic loops and the control of the average fluid pressure by the sympathetic loop. Initially the sympathetic transmitter peak corresponds approximately with the parasympathetic activity peak in the middle of the heart interval. The sympathetic transmitter trough is after the systolic peak because the sympathetic transmitter rise time follows the Windkessel time constant whereas the fall time is due to the metabolic time constant which is longer. The sino-atrial time constant is controlling the initial phase advance. The sympathetic transmitter is decaying at this point to its minimum, and so is the parasympathetic influence which is also attenuated by the phase effectiveness curve. The parasympathetic loop controls the middle of the heart interval due to the $.5 \mathrm{sec}$ delay from the start of the heart interval and the effect of the phase effectiveness curve increasing at this point. As the sympathetic transmitter metabolic time constant is longer the parasympathetic influence decreases faster and the sympathetic transmitter controls the end of the interval.

As the parasympathetic loop gain increases, the interval time becomes longer. The diastolic pressure therefore decreases as much as the sympa- 


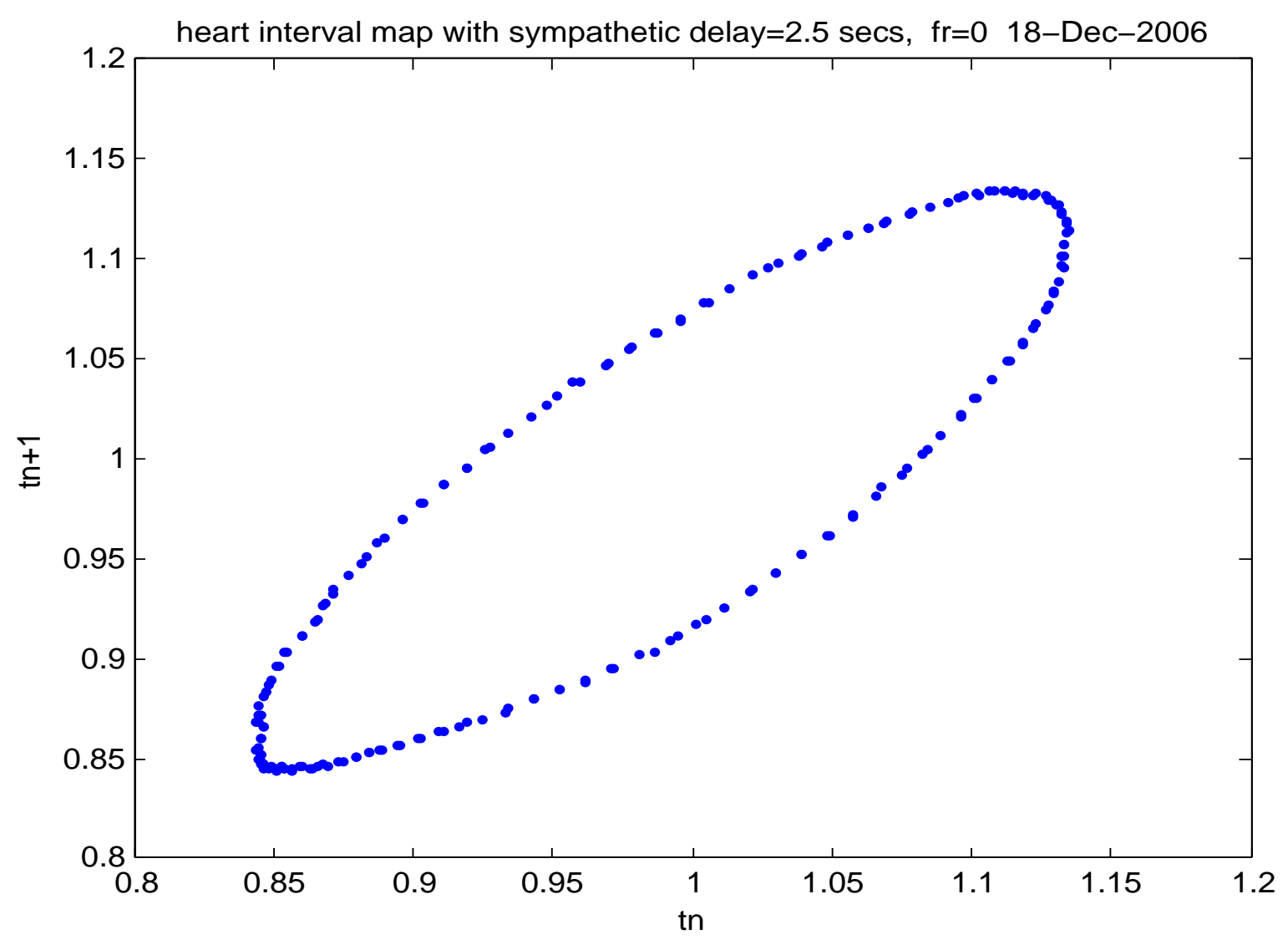

Figure 4.2: heart interval return map 


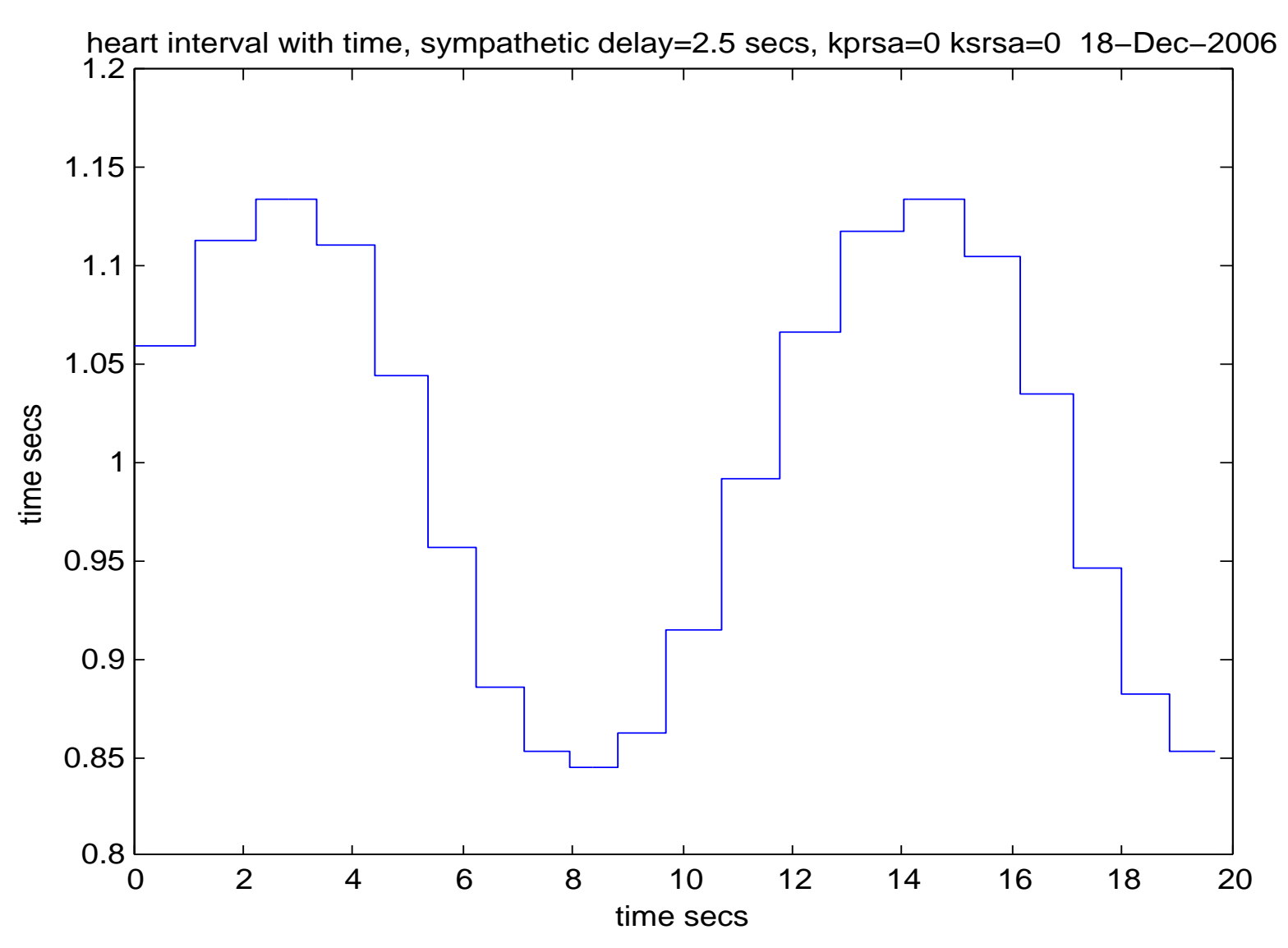

Figure 4.3: time depiction of heart interval length 


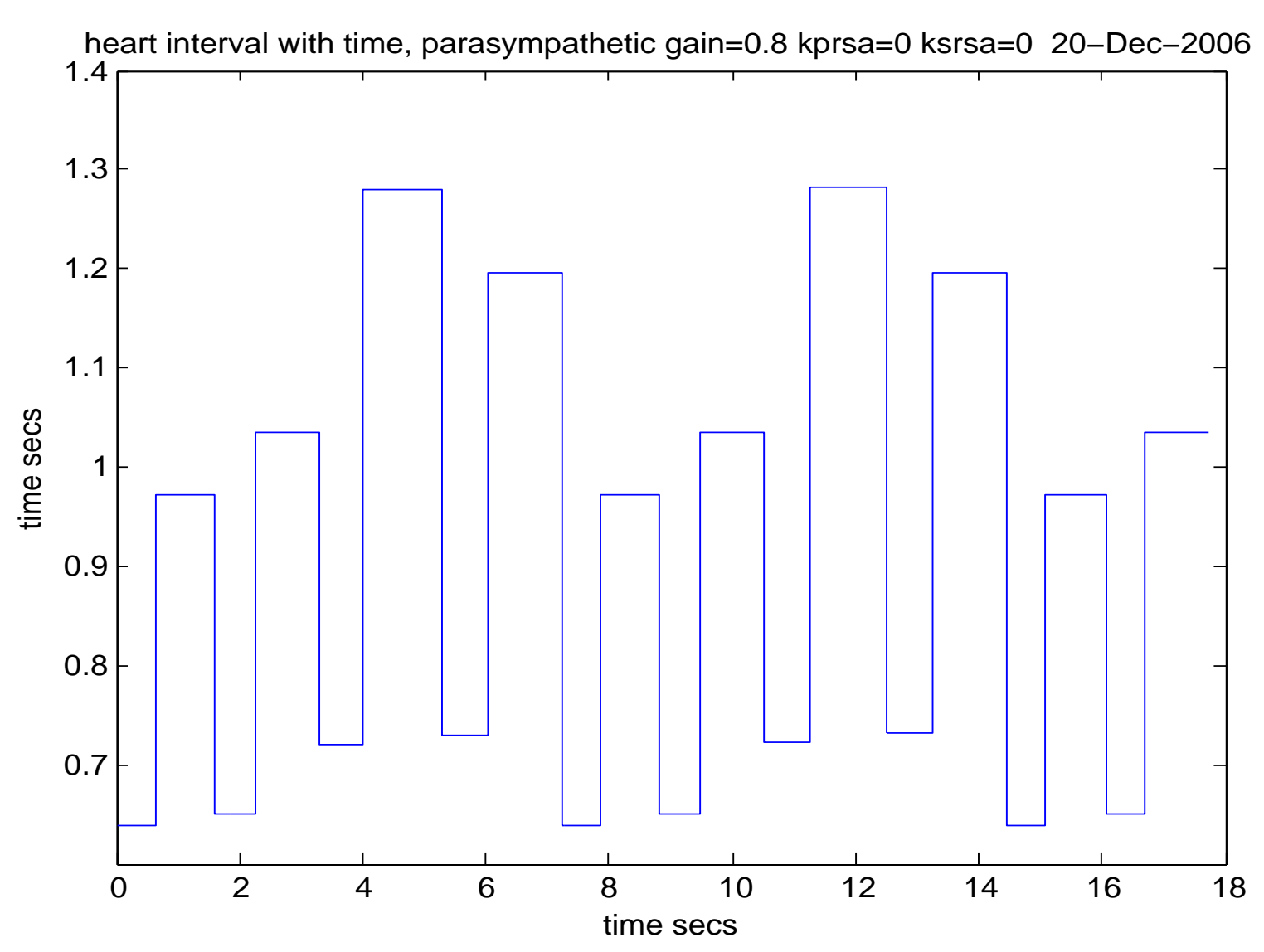

Figure 4.4: 8:1 entrainment of alternans arrhythmia 
thetic control loop allows because it needs a small difference from the set point to counteract the effect of the increased parasympathetic activity.

At the bifurcation point the interval has lengthened, and the heart phase rises very sharply at the end of the interval due to the stronger influence of the sympathetic transmitter. The sympathetic activity threshold now corresponds to heart phase $\phi=0$ and corresponds to an inflection in the decay of the sympathetic transmitter

As the sympathetic activity threshold crosses into the $n-1$ interval the following sympathetic activity peak is adding to the sino-atrial node time constant to shorten the interval $n$ as it moves away from the opposing parasympathetic influence. Additionally the contractility $n$ is decreasing as the minimum of the sympathetic transmitter moves closer to zero phase, so decreasing the subsequent parasympathetic lengthening influence in interval $n$. The $n-1$ interval is still responding to the increased parasympathetic loop gain but less so due to the effect of the difference in time constants, but this is also counteracted by the overall decrease in the baroreflex influence being dominated by the sino-atrial time constant. As the interval $n$ shortens and the contractility $n$ decreases, the following interval $n+1$ has its associated $n-1$ sympathetic activity shift back into the $n+1$ interval more under counteracting effect of the $n+1$ parasympathetic activity so adds to the lengthening effect on the $n+1$ interval. The long interval therefore now has two sympathetic activity threshold crossings and the short interval none.

As the average pressure decreases the sympathetic loop gain increases due to the reduction in amplitude compression of the $S$ curves and oscillation occurs in the sympathetic loop.

\subsubsection{Frequency response of heart system}

The frequency response of the heart interval length to the RSA or centrally coupled noise was studied. This was found by using a low level of excita- 
tion in the RSA terms of the equations (4.1)-(4.13). The Fourier spectrum is frequency modulated and the side bands of the carrier frequency give the magnitude of the deviation of the heart interval frequency depending on the excitation frequency provided the excitation is small and the FM spectrum has only 1 side band. The frequency deviation should be much less than the modulation frequency for this. See M. Schwartz p273, [34]. So one method of finding the frequency response is to examine the spectrum around the carrier frequency at low coupling to determine the frequency modulation of the carrier.

Alternatively as the heart interval is a frequency modulated delta function sequence, the modulation spectrum should be present in the base band. Intuitively this is seen by observing that integrating a frequency modulated delta function sequence at a time constant longer than the carrier period (and shorter than the modulation period) should give a signal proportional to the modulating signal. The heart interval signal is analysed by considering it to be a delta function sequence frequency modulated by the RSA.

The frequency modulation model of the HRV is a simplification. HRV is formalised by the Integral Pulse Frequency model (IPFM) which is a relaxation oscillator model in which both the integration rate and the threshold are modulated. This corresponds to the different effects of parasympathetic and sympathetic influence due to their different chemical actions. The Poincaré return time is most often treated as being due to a constant frequency but clearly any model is possible for the phase advancement between the Poincaré surfaces. [35],[36],[37],[38].

The spectrum was found by exciting the RSA terms in the system with a random constant value for each interval. In the frequency spectrum the input to the RSA is therefore white noise with a constant frequency spectrum and it is the power spectrum of the output that is calculated. The coupling was small $=.04$ to avoid the effect of nonlinearity. 100000 heart intervals were calculated and the Fourier power spectrum computed. If 
the total length of the heart intervals is $T$ then the components of the spectrum at multiples of $\frac{1}{T}$ correspond to Fourier series coefficients. Logically whatever is the signal outside of the rectangular window it could be a periodic function of the signal inside the window and in that case the spectrum would be a Fourier series with components at $\frac{1}{T}$. The Fourier series of the periodic function does exist but the transform does not, or it is a delta function sequence weighted by the Fourier coefficient, as the integration over 0 to $T$ is multiplied by $\Sigma_{n=-\infty}^{\infty} \mathrm{e}^{-j n \omega T}$ to obtain the transform of the periodic signal. Multiplying the periodic signal by a $T \mathrm{e}^{-j \frac{\omega T}{2}} \frac{\sin \frac{\omega T}{2}}{\frac{\omega T}{2}}$ to obtain the time frame, the zeros of the $\frac{\sin (x)}{x}$ function coincide with the other Fourier series components so the value of the time interval spectrum at the Fourier component frequency must be that of the Fourier series component amplitude. The Fourier components are what is meaningful because knowledge of these allows the signal to be completely reconstituted. The frequency spectrum values of the Fourier Transform between those corresponding to the Fourier series coefficients are sums of values of a $\frac{\sin (x)}{x}$ function weighted by the Fourier coefficients.

It is seen that the increase in the spectrum envelope at low frequencies is due to the finite time interval length and is weighted by the $\frac{1}{x}$ term giving a linear increase in the log spectrum. This gives a time average of the Fourier coefficients at a resolution of $\frac{1}{T}$. This is a high resolution for a long period $T$ but does not average the noise for each coefficient effectively. An ensemble average of many shorter time lengths is used to find more accurate values of the spectrum at the components of the Fourier series but at a lower frequency resolution. So a time interval length of about 8192 seconds was used with a Hanning window $H(t)=1-\cos \left(\frac{2 \pi t}{T}\right)$ to reduce leakage of the rectangular window due to the discontinuity at each end, as well as using an integral number of the average heart interval length for the time interval to reduce the discontinuity, which gives an ensemble of about 120 time intervals. The power spectrum is found as the magnitude squared of the Fourier transform of the heart interval data as 
the autocorrelation of the heart interval data is difficult to calculate being based on delta functions. As the heart intervals are discrete the system is a sampled data system. The sampling frequency is the number of intervals $n$ divided by the total length of the intervals $T$, so $\frac{n}{T}$. So the spectrum is repeated at intervals of $\frac{n}{T}$.

One method of detection of the modulation would be to phase lock the sampling frequency to the data and determine the time deviation for each interval exactly. The spectrum is then found from the length of each deviation equiplaced at the sampling frequency, and applying a DFT. This is equivalent to simply transforming the heart interval data placed at the intervals of the sampling frequency. As discussed earlier this would be an approximation. This would assume that the modulation was being sampled at the sampling frequency whereas clearly the time interval length is determined instantaneously by the integration rate and the threshold at the end of the heart interval, so the modulation is sampled at this time. The data could be made equispaced by assuming a model for the modulation signal and interpolating. Akselrod and Keselbrener [39], use a DFT model of varying resolution to interpolate the time interval data.

As the system is a frequency modulated pulse train the spectrum also exists in the base band. The spectrum was directly computed by a piecewise integration of the Fourier Transform so each interval was represented by a $\delta$ function spaced by the time intervals. In figure (4.5) the response of the sympathetic loop can be seen in the response as the peak at $.1 \mathrm{~Hz}$. It was seen that noise coupled into the sympathetic loop was subject to the metabolic dynamics of the sympathetic transmitter and consequently the higher frequency band was attenuated. This is illustrated in figure (4.6) when the parasympathetic loop coupling is set to zero. In physiological data a parasympathetic response would also be noticeable but also a roll off of the response and this seems to indicate that the sympathetic loop is subject to more noise and that the parasympathetic loop has higher gain than the nominal value of the model. As the system is a sampled data 


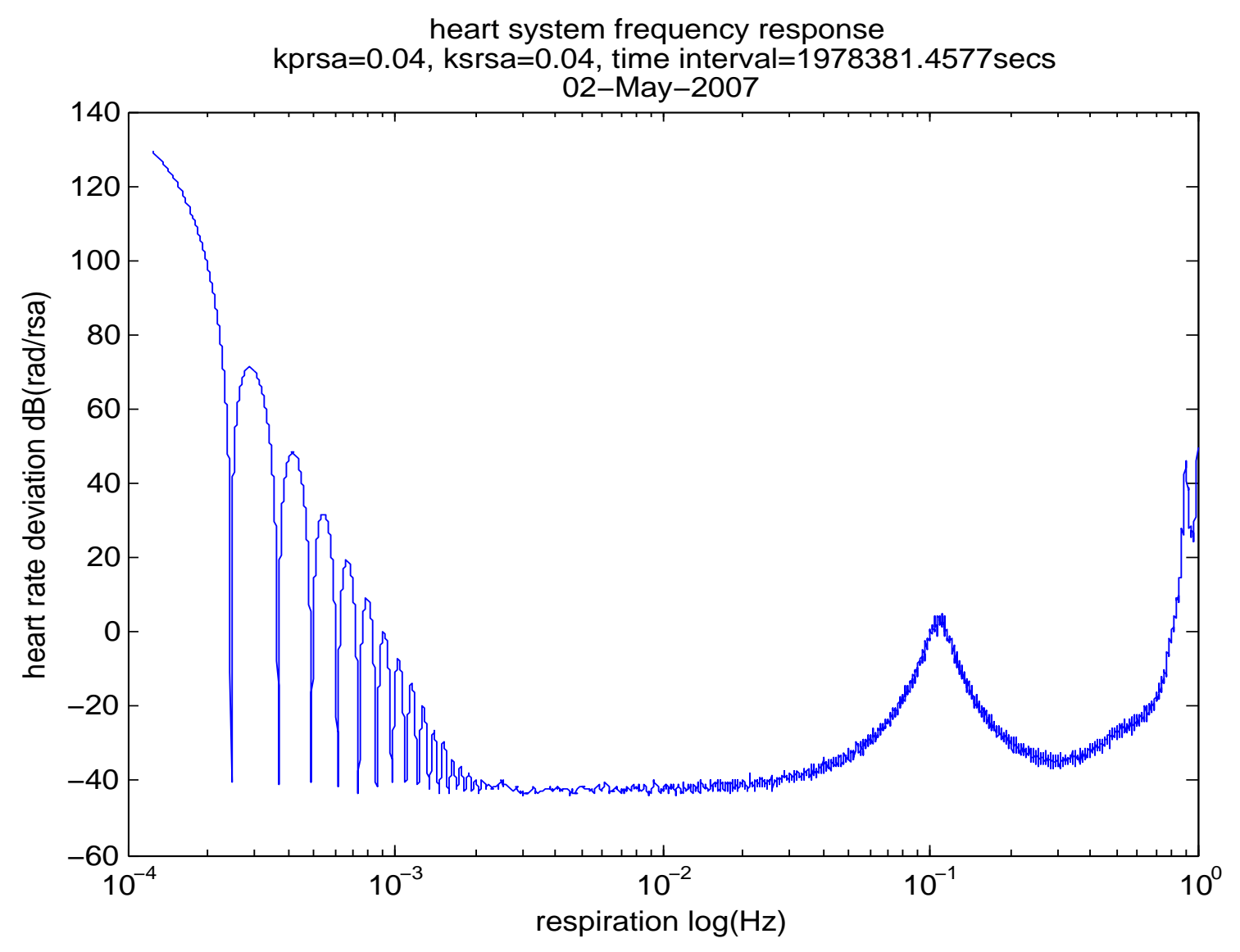

Figure 4.5: frequency response of heart system 


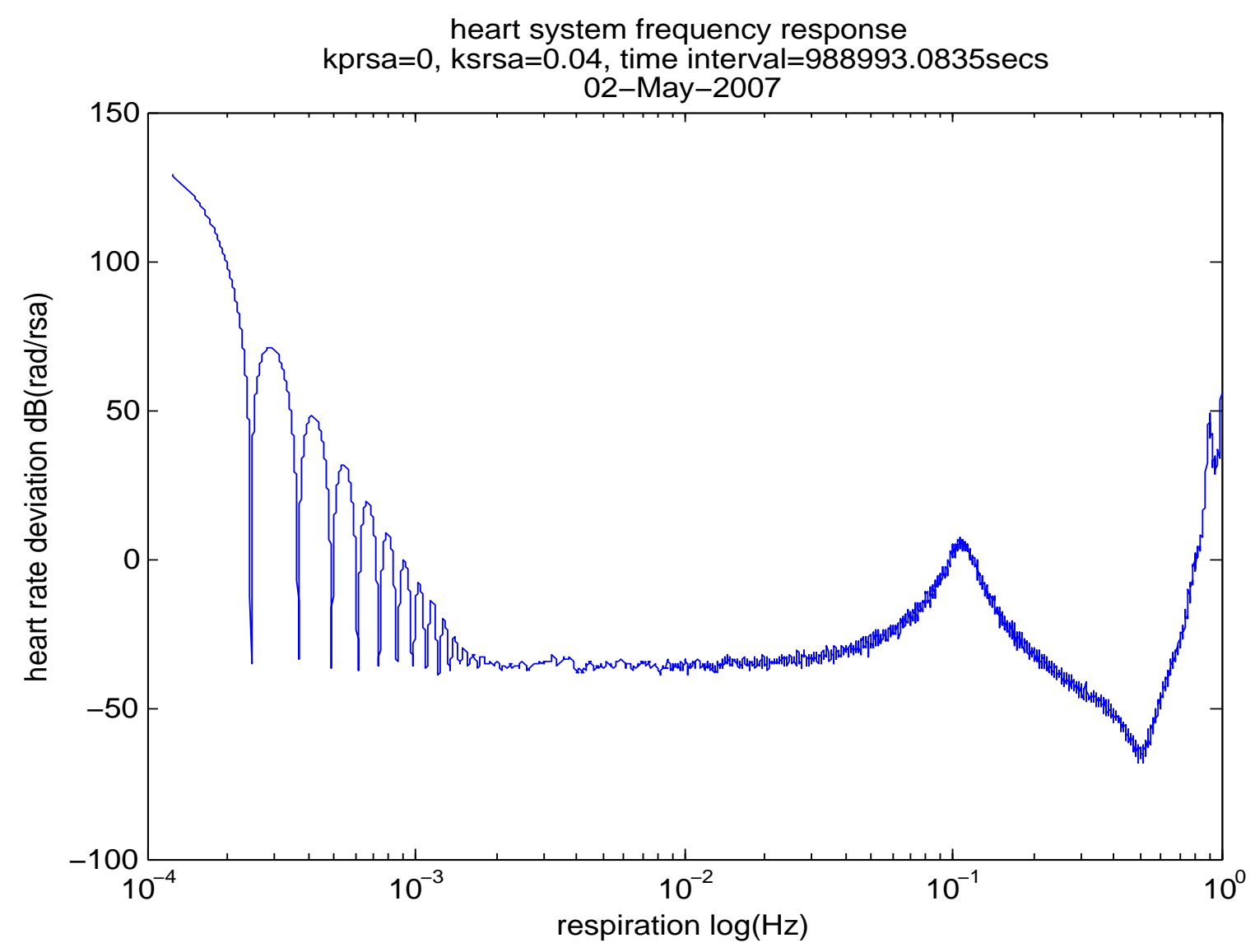

Figure 4.6: response with parasympathetic coupling set to zero 
system aliasing effects are apparent around $f_{c} / 2$ that is $.5 \mathrm{~Hz}$. The average interval length of the data is $\sim .99$ secs so the fundamental frequency is $\sim 1.01 \mathrm{~Hz}$. 


\section{Chapter 5}

\section{Coupling in the Cardiovascular System}

\subsection{Introduction}

Considering the cardiovascular system as two coupled oscillators the simplest mathematical model might be:

$$
\begin{aligned}
& \dot{\phi}_{1}=\omega_{1}+\varepsilon f_{1}\left(\phi_{1}, \phi_{2}\right)+\xi_{1}(t), \\
& \dot{\phi}_{2}=\omega_{2}+\varepsilon f_{2}\left(\phi_{1}, \phi_{2}\right)+\xi_{2}(t),
\end{aligned}
$$

where $\omega_{i}$ are the frequencies of each oscillator in the absence of coupling or noise, $f_{i}$ the coupling functions periodic in $\phi_{1}, \phi_{2}$, and $\xi_{i}$ noise or higher dimensional dynamics of the oscillator system which have the effect of noise such as chaos or toroidal oscillations. The trajectories $\phi_{i}$ have a zero Lyapunov exponent and are defined on the real line, that is they are a lift of an oscillator defined on an interval. The coupling functions need not be identical so that the coupling is asymmetrical. A driven oscillator system has one of the $f_{i}$ zero and so a uniform phase advance for that oscillator in the absence of noise. The dynamics of the phase difference depend on the nature of the coupling. 
For synchronisation the coupling must be a function of the phase difference $\phi_{d}=m \phi_{1}-n \phi_{2}$, where $m, n$ are integers, so that an equilibrium point of the phase difference dynamics is a fixed phase relationship between $\phi_{1}, \phi_{2}$. i.e. see section (3.3). Consider the phase difference dynamics $m \dot{\phi}_{1}-n \dot{\phi}_{2}=\dot{\phi}_{d}$. If the coupling functions $f_{i}$ are not entirely a function of the phase difference $\phi_{d}$ then the dynamical equation is effectively nonautonomous driven by terms in $\phi_{1}$ and $\phi_{2}$, possibly nonlinear. As long as the variation in the phase difference does not exceed $\pm \pi$ the signal will remain in synchronisation. As elaborated in section (3.3) the phase difference $\phi_{d}$ is not strictly a constant because of the non-autonomous terms. Expressing the phases relative to a common zero let $\phi_{i}^{\prime}=\phi_{i}+\phi_{i}^{c}$. Writing the phase difference $m \phi_{1}^{\prime}-n \phi_{2}^{\prime}=m\left(\phi_{1}+\phi_{1}^{c}\right)-n\left(\phi_{2}+\phi_{2}^{c}\right)=0$ shows that $m \phi_{1}-n \phi_{2}=n \phi_{2}^{c}-m \phi_{1}^{c}$ a constant for synchronisation, so linearly translated coordinates are identical at synchronisation, and that if $\phi_{d}$ varies by more than $\pm \pi$ then a cycle has slipped. When synchronisation is maintained, $\phi_{d}$ is periodic over the period of the synchronisation $\left(n T_{1}=m T_{2}\right)$.

Considering the symmetry of the coupling functions, a function can be decomposed into an odd part $f_{o}$, where $f_{o}(x)=-f_{o}(-x)$, and an even part $f_{e}$, where $f_{e}(x)=f_{e}(-x)$. Even parts of the coupling functions will cancel when differenced so that the phase difference dynamics are odd or have an even part if the coupling is asymmetrical. An even part in the phase difference vector field shifts the equilibrium points from the nominal zero phase difference of an odd function as it is equivalent to a phase shift of the coupling function. It is seen that asymmetrical coupling does not change the nature of the dynamics of the phase difference so that a driven oscillator system is a good model for studying bifurcations of coupled oscillators.

Generally the coupling term $k\left(\phi_{1}, \phi_{2}\right)$ of the phase difference dynamics $\phi_{d}=\phi_{1}-\phi_{2}$, is a function of $\phi_{1}$ and $\phi_{2}$. Suppose it is continuously differentiable $C^{1}$ on $T^{2}$, so $2 \pi$ periodic in $\phi_{1}, \phi_{2}$, and so can be expressed as a two dimensional Fourier series. Therefore all trignometric terms in $m \phi_{1} \pm n \phi_{2}$ are possible. The coefficient of the respective $m \phi_{1}-n \phi_{2}$ difference terms 
gives the coupling coefficient for a particular $\frac{m}{n}$ ratio and the remaining terms of the series are synchronous with the period of the synchronisation when the system is synchronised, so their effect averages out to zero over this period according to the averaging theorem (see F. Verhulst [25] p149). When the system is not synchronised then the oscillators must be at their nominal frequencies as the averaging theorem is valid for quasi-periodic frequencies also. Higher order terms at multiples of the locking ratio result in a non-sinusoidal coupling function.

Non-isochronicity in an oscillator (section (3.2)), can be expressed by another dimension $r$ (or dimensions), which gives higher order dynamics to the synchronisation process. For example an electronic phase locked loop is generally second order or higher [40]. In this case the oscillators are based on the phase model but the coupling is via a dynamical system. So the coupling function is $f(r)$ and $\dot{r}=g\left(r, \phi_{d}\right)$. Generally the coupling is $f_{i}\left(r, \phi_{1}, \phi_{2}\right)$, and $\dot{r}=g\left(r, \phi_{1}, \phi_{2}\right)+\xi_{r}(t)$. A higher dimensional oscillator (non-chaotic) is necessarily of the nature of a limit cycle attractor so the synchronisation properties must depend on the coupling on the limit cycle.

\subsection{A Phase locked loop model of Cardiovascular system synchronisation}

It can be seen that while the sino-atrial node can be modelled as a simple relaxation oscillation, the heart oscillator is made non-isochronous by virtue of the baroreceptor loop. This loop modifies the progression of the phase depending on the pressure in the aorta. Considering a 3D phase map of the heart oscillator system, cylindrical co-ordinates $r, \theta, z$ could be used, where $\theta$ is the sino-atrial phase, $r$ is the pressure in the aorta, and $z$ is the time axis which makes the system autonomous. An increase in pressure slows down the phase progression and lengthens the heart inter- 
val. So the system is non-isochronous in the $r$ dimension and responds to perturbations of pressure. As the sympathetic loop has a higher gain than the parasympathetic loop it is the more significant for second order effects on the RSA. So sympathetic transmitter concentration is the most significant variable in expressing the non-isochronicity of the system, that is the dynamics of the level of the sympathetic transmitter have a second order effect on the RSA. It can be seen (e.g. from figure (4.1), and equation (4.3)) that the level follows the respiration with some delay and the diastolic pressure is directly related to the level of sympathetic transmitter.

The heart oscillator can be compared to the electronic frequency controlled oscillator in a 2nd order phase-locked loop [40]. As a system the driven 2nd order phase locked loop consists of two 1D oscillators and a coupling function which is a first order system of the phase difference. So as a $3 \mathrm{D}$ system the dynamics are restricted by the oscillator subsystems and the extra dimension gives 2 nd order dynamics to the synchronisation. Chaotic behaviour must not be possible as the Lyapunov exponents of the oscillators are zero which restricts the remaining Lyapunov exponent to be $<0$ for bounded behaviour. But it is possible to generate chaotic behaviour by perturbing the driving signal.

An electronic phase locked loop is characterised by a difference between the capture range, that is the level of detuning between the oscillator centre frequency and the input signal frequency at which the oscillator will lock for all initial conditions of oscillator frequency and phase, and the hold range, that is the detuning over which the oscillator will remain in lock from an initial locked state, as the input signal frequency is changed.

A phase locked loop has coupling dependent on the gain of the system which is a parameter, whereas in the cardiovascular system the RSA is relatively weakly coupled and fixed by physiological considerations. In a phase locked loop the phase difference between the oscillator and the driving signal is detected explicitly by a phase detector giving a sinusoidal, or triangular, phase difference characteristic, rather than through a nonlinear 
process as in the cardiovascular system. It is a coupled oscillator system as the voltage controlled oscillator is driven by phase difference dynamics.

A similar mechanism applies to the RSA input to the heart oscillator. In a locked state there is a constant phase difference which is necessary to hold the frequency controlled oscillator at the driving frequency. The RSA input directly modulates the heart interval length via the sino-atrial nervous input proportionate to the depth of the RSA, and the depth of the RSA is a function of the respiration phase. Without the nonlinear expression of sino-atrial node phase through the gain of the phase responsiveness curve $F(\phi)$, as in figure (5.3), the effect of the respiration would simply be to modulate the heart interval length, but the product term of heart phase and respiration phase generates a change in heart frequency and therefore heart interval length, proportionate to the phase difference. The effect of the perturbations of the heart interval by the RSA is to cause changes in pressure and this is therefore counteracted by the nonisochronicity expressed by the parasympathetic and sympathetic loops which degenerate the open loop effect of the RSA.

Sum and difference terms of heart phase and respiration phase are generated by the multiplication of the phase reponsiveness curve $F(\phi)$ with the sino-atrial input due to respiration. The rational relationship of the oscillators is fixed by the multiple of the harmonic terms of phase difference. A possible complication in the system is the baro-receptor S-curve which compresses higher pressures so that the effect of the respiratory influence is eliminated while the pressure is high, and only acts at the end of the heart interval as the pressure decreases. Considering the delay of the parasympathetic metabolism (.5 secs) it can be seen that as the nervous influence due to pressure comes out of compression at a time related to the peak pressure the falling edge of the pressure has the respiratory influence imposed on it as well as variations in pressure due to the action of the cardio-vascular system. This edge then falls within the most dynamic part of the phase responsiveness curve. The effect would cause the signal 
to the sino-atrial node to be of the form of a pulse modulated in width.

In practice it was found that there was little compression from the Scurves, so normally the system is operating on a nearly linear part of the curve. It did seem that the S-curves were ineffectual when using the nominal parameter values, so it is possible that the validity of these values should be verified as they only seem to operate for very high pressure levels. Increasing the compression of the S-curves did result in the locking zones increasing for low levels of coupling but diminishing as coupling increased as the respiratory influence saturated the S-curves. Equally the linearity of the S-curves is illustrated by the dependance of the phase locking on the phase responsiveness curve $F(\phi)$. Replacing the phase responsiveness curve by a constant, the locking zones diminished in width by $25 x$. So the multiplication of the phase responsiveness curve by the coupled respiration is nominally the only nonlinearity leading to phase locking. The various S-curves are operating in a nearly linear region and must account for the small residual synchronising effect.

For the sympathetic loop there is no phase responsiveness curve as the metabolism and effect of the neurotransmitter is different, so synchronisation depends on the para-sympathetic baroreflex loop. However there is cross coupling between the sympathetic loop and the para-sympathetic loop at the sino-atrial node as shown by the product term $f_{s} f_{p}$ in equation (4.4) for heart phase in the model, so there is a second order dependence of synchronisation on the sympathetic loop. The terms are of the form $f_{p}=1-F(\phi) k_{p}\left(v_{b}(t)+x(\theta)\right), f_{s}=1+k_{s}\left(v_{s}^{(0)}-v_{b}(t)+x(\theta)\right)$, where $F(\phi)$ is the phase responsiveness curve, $x(\theta)$ is the respiratory influence (expressed by the $k_{p}^{r}\left|\sin \left(\pi f_{r} \mathrm{t}+\Delta \phi_{p}^{r}\right)\right|$ in eqns (4.2),(4.3)), $v_{s}^{(0)}$ is the sympathetic transmitter threshold, and $v_{b}(t)$ expresses that the decay of the pressure is approximately proportional to the interval phase.

A second order phase locked loop contains a low pass filter which modifies the dynamics of the loop. The low pass filter increases the order or dimension of the system. The dimension of the heart oscillator sys- 
tem is also increased by the time delay of the reflex loop which causes the oscillator to be an infinite dimensional system. Whether the higher dimensions are significant will depend on the magnitude of the higher order Lyapunov exponents. From a practical point of view the significant difference between an electronic phase locked loop and the heart system is that the low pass filter, as well as modifying the dynamics of the phase locked loop reponse also removes artifacts of the phase detection process. In the electronic phase locked loop this is a $2 f$ component. The sino-atrial node phase responsiveness is quite nonlinear so many components are present besides the detected phase difference. These components cause fluctuations in the synchronizing force which lead to jitter in the phase difference between the two oscillators. Whether a phase locking zone exists will depend on the magnitude of the jitter.

Figure (5.1) shows a simplified heart system represented as a phase locked loop. In the figure $K_{r s a}$ is the respiration coupling and $K_{h}$ is the gain of the heart oscillator frequency response. $F(\phi)$ is the phase responsiveness curve. The heart oscillator is affected through the parasympathetic control. The heart oscillator system counteracts the disturbance caused by the respiratory influence on the pressure through the parasympathetic and sympathetic loop and through the peripheral resistance after some delay depending on the path. This causes the locking zone width to be a function of respiration frequency although this frequency responsiveness is not shown on figure (5.1). It seems that the parasympathetic loop which acts within a heart interval is part of the heart contractility subsystem. It seems to be the sympathetic loop that adjusts the heart interval and peripheral resistance depending on fluid pressure in the aorta and other arteries.

The locking dynamics can be expressed as a first order differential equation. Consider the $1: 1$ locking region as other ratios are similar but occur through higher harmonics in the phase responsiveness curve $F(\phi)$. The phase responsiveness curve can be approximated by the function $A-$ 


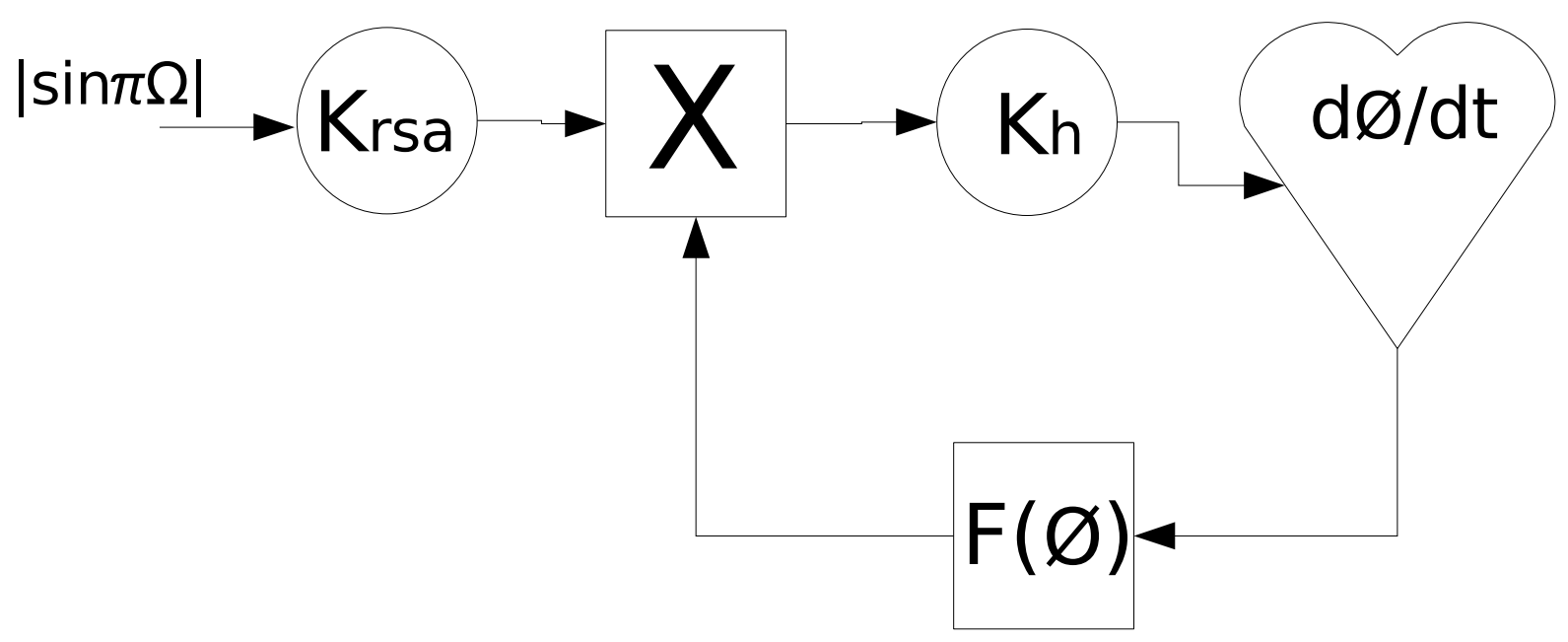

Heart system first order phase locked loop

Figure 5.1: heart system first order phase locked loop 
$B \sin \left(\phi_{h}\right)$ for the $1: 1$ ratio. $A=.03222, B=.10428$. These values are obtained by solving in Maple with the same ordinate for the zero crossing and the same value at the ordinate of the peak value of the phase responsiveness curve. The fundamental component of the respiratory influence function $\left|\sin \left(\pi f_{r} t\right)\right|$ (from eqns $(4.2,4.3)$ ) is $-\frac{4}{3 \pi} \cos \left(\omega_{r} t\right)$. From the diagram 5.1 , let

$$
\begin{aligned}
& \frac{d \theta_{r}}{d t}=\omega_{r}, \\
& \frac{d \phi_{h}}{d t}=\omega_{h} \quad+K_{r s a} K_{h} \frac{4}{3 \pi} \cos \left(\theta_{r}\right) F\left(\frac{\phi_{h}}{2 \pi}\right),
\end{aligned}
$$

be the equations of the pll illustrated, and using the fundamental component of the respiratory influence, and where $\phi_{h}, \theta_{r}$ are the heart phase and respiration phase respectively, $\omega_{h}$ is the nominal heart frequency and $\omega_{r}$ is the frequency of respiration. Taking the difference of the two equations (5.2),(5.3), and using the above approximation for the phase responsiveness curve $F(\phi)$, let

$$
\frac{d\left(\phi_{h}-\theta_{r}\right)}{d t}=\omega_{h}-\omega_{r}+B K \sin \left(\phi_{h}-\theta_{r}\right)
$$

where $K=\frac{2}{3 \pi} K_{r s a} K_{h}$. Let $\phi_{d}=\phi_{h}-\theta_{r}$ be the phase difference, then the dynamics of the RSA locking are:

$$
\frac{d \phi_{d}}{d t}=\Delta \omega+B K \sin \left(\phi_{d}\right)
$$

It is seen that a time $=T$ map at the frequency of the respiration would be a sine map. For this first order phase locked loop there is an analytical solution:

$\phi_{d}(t)=2 \cdot \arctan \left(\frac{\tan \left(\frac{1}{2} t \sqrt{\Delta \omega^{2}-(K B)^{2}}+\frac{1}{2} \phi 0 \cdot \sqrt{\Delta \omega^{2}-(K B)^{2}}\right) \sqrt{\Delta \omega^{2}-(K B)^{2}}-K B}{\Delta \omega}\right)$,

where $\phi 0=\frac{2 \cdot\left(\arctan \left(\frac{\Delta \omega \cdot \tan (\phi(0) / 2)+K B}{\sqrt{\Delta \omega^{2} B^{2} K^{2}}}\right)\right.}{\sqrt{\Delta \omega^{2} B^{2} K^{2}}}$.

Figure 5.2 shows the plot of the vector field of the first order phase locked loop normalised wrt $K B$. The equilibrium points occur at the intersection with the $\phi$ axis. The intersection with positive slope has isoclines 

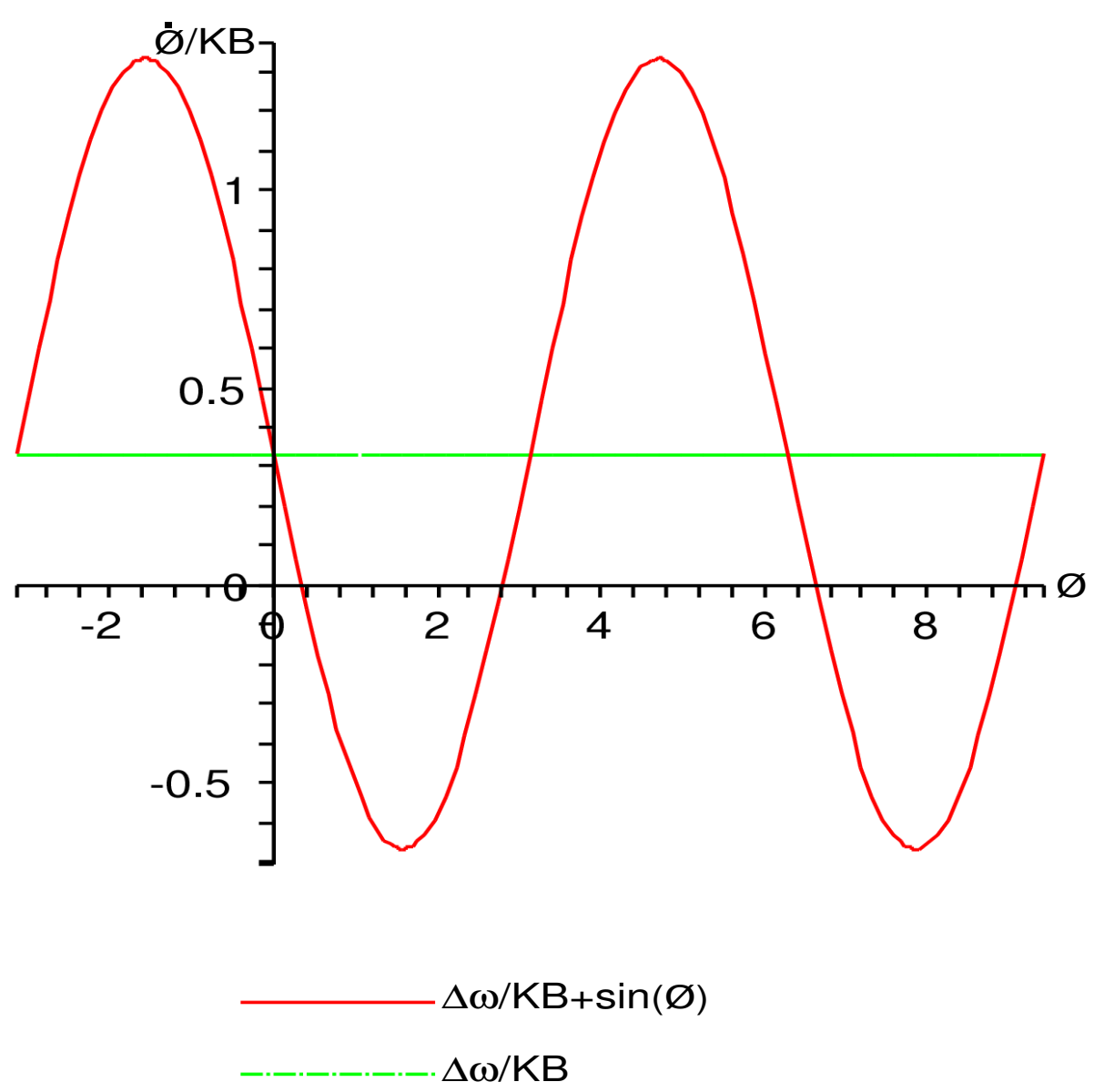

Figure 5.2: phase line plot for first order phase locked loop 
away from the point so it is the intersection with negative slope that is stable. For locking $\left|\frac{\Delta \omega}{K B}\right|<1$ therefore both the pulling range and the hold range are $\pm K B$ radians.

\subsection{The Synchronization mechanism in the Cardiovascular system}

It is clear that the respiration affects the heart interval as a function of the phase difference between the heart interval and the respiration cycle, when one considers the phase responsiveness curve of the sino-atrial node. This curve expresses the phase of the heart oscillator by sensitivity of the rate of phase advance to parasympathetic input depending on the phase of the oscillator. Synchronisation requires that the coupling is a function of the phase difference between the two oscillators so that the phase difference is an autonomous dynamical system.

For the model equations (4.1)-(4.13) the phase responsiveness curve $F(\phi)$ is as given by Seidel and Herzel ([31]) and is a curve fitted to theoretical data from an ionic model of the sino-atrial node.

Clearly a corresponding $m \phi_{1}-n \phi_{2}$ term has to be generated for the $\frac{m}{n}$ locking region. Generally the nonlinearities of a system must generate the required phase difference terms for a system driven by a single tone. For a more complicated driving signal such as the respiration in the cardiovascular system there are additional harmonics in the driving signal as well which explicitly generate higher order phase differences. In the model the coupling function $f(\phi, \theta)=\left|\sin \left(\frac{\theta}{2}\right)\right| F(\phi)$.

The half sine function $f(t)=\left|\sin \left(\frac{\pi}{T} t+\Delta\right)\right|$ has the Fourier series:

$$
f(t)=\frac{2}{\pi}-\frac{4}{\pi} \cdot\left(\frac{\cos 2\left(\frac{\pi}{T} t+\Delta\right)}{1 \cdot 3}+\frac{\cos 4\left(\frac{\pi}{T} t+\Delta\right)}{3 \cdot 5}+\frac{\cos 6\left(\frac{\pi}{T} t+\Delta\right)}{5 \cdot 7}+\cdots\right),
$$

where $T$ is the respiration period. The phase responsiveness curve $F(x)=$ 


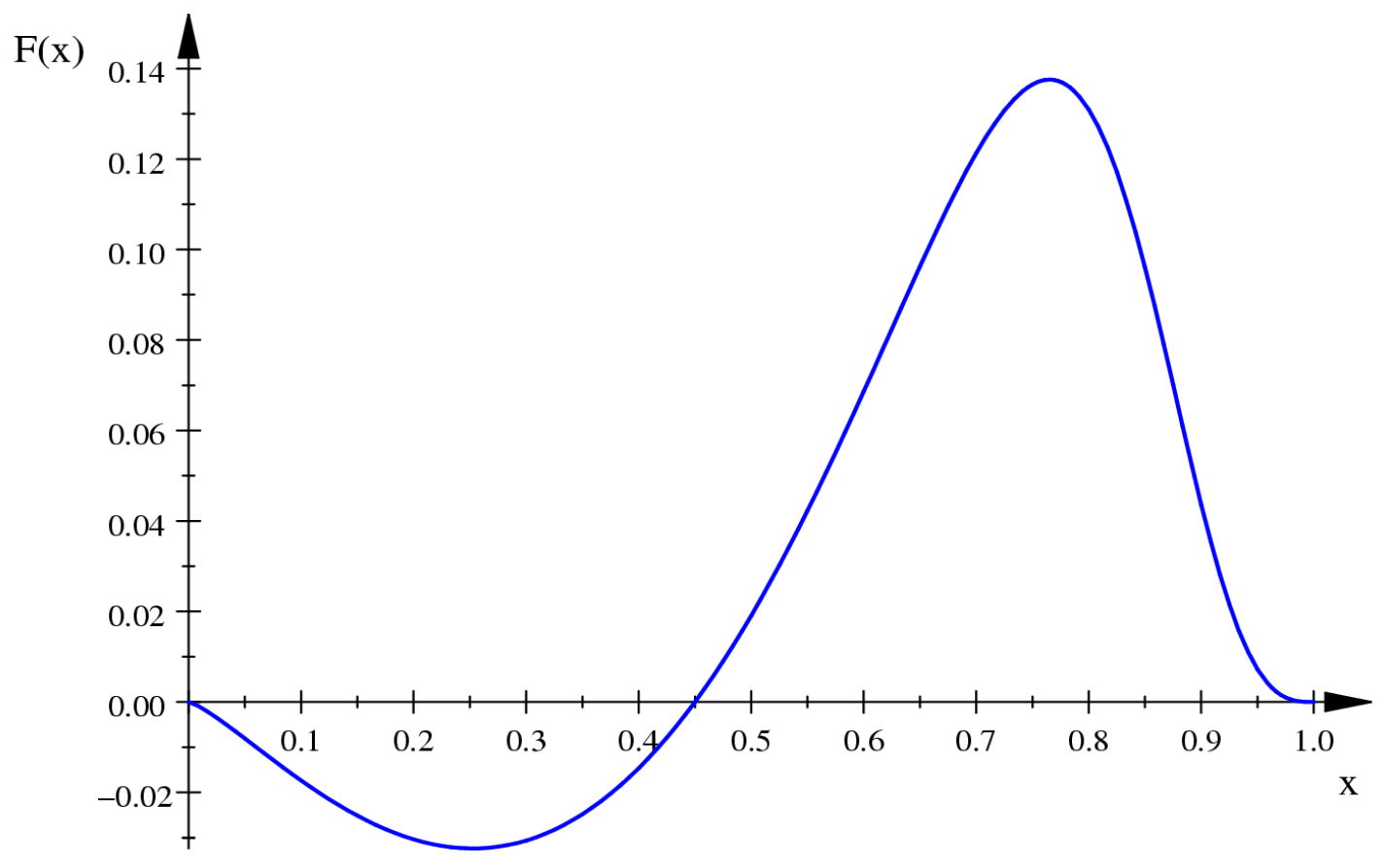

Figure 5.3: heart oscillator phase responsiveness curve 
$x^{1.3}(x-0.45) \frac{(1-x)^{3}}{(1-.8)^{3}+(1-x)^{3}}$ is multiplied with the perturbation due to respiration to generate phase difference and sum components which modulate the heart rate. The phase responsiveness curve can be expressed in a Fourier series:

$$
F(x)=\frac{a_{0}}{2}+\sum_{n=1}^{\infty}\left(a_{n} \cos \left(\frac{2 n \pi x}{a T}\right)+b_{n} \sin \left(\frac{2 n \pi x}{a T}\right)\right),
$$

where $a$ gives the heart interval period as a fraction of $T$. The first five coefficients are

\begin{tabular}{|l|l|l|}
\hline $\mathrm{n}$ & $\mathrm{a}_{n}$ & $\mathrm{~b}_{n}$ \\
\hline 0 & 0.0289 & \\
\hline 1 & -0.0040 & -0.0383 \\
\hline 2 & -0.0113 & 0.0016 \\
\hline 3 & -0.0022 & 0.0047 \\
\hline 4 & 0.0007 & 0.0024 \\
\hline 5 & 0.0009 & 0.0008 \\
\hline
\end{tabular}

Figure 5.3 shows the phase responsiveness curve. Figure 5.4 shows the respiration influence on the sino-atrial node assuming that the respiration perturbs the autonomic system influence linearly. Figure 5.5 shows how the heart interval length varies vs respiration phase for a 5:1 heart rate to respiration rate ratio. The RSA influence occurs via the parasympathetic loop so an increase in influence lengthens the heart interval. So the heart interval length relative to the respiration phase varies over a range of $10 \%$. This effectively gives the map for the heart interval length as a function of respiration phase.

The first terms of the series (5.7), (5.8) are multiplied together to show the components that are present to modulate the heart interval time. When there is synchronisation $a=\frac{m}{n}$, so there are $m$ respiration cycles to $n$ heart 


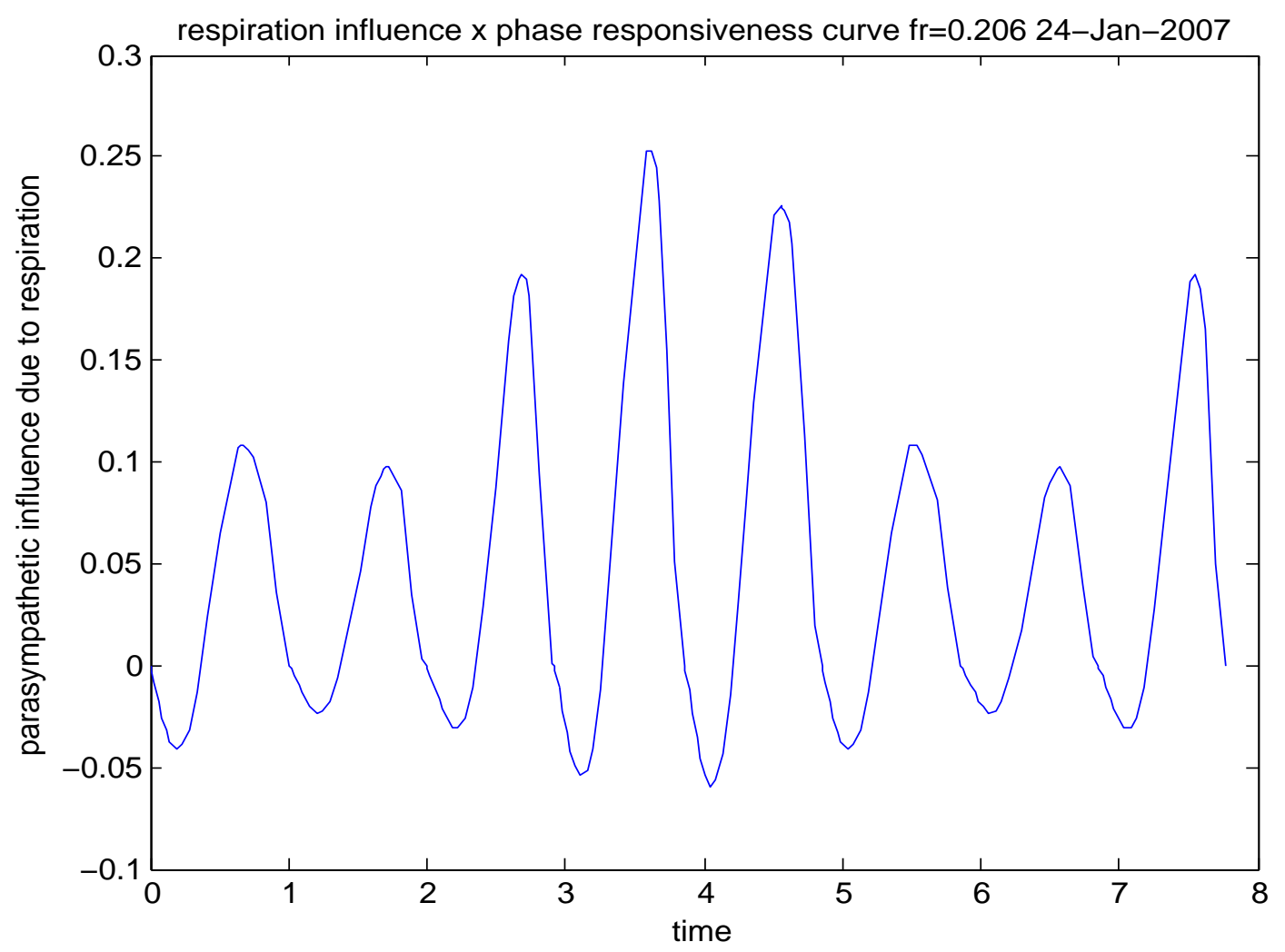

Figure 5.4: Effect of respiration on heart rate calculated from model data 


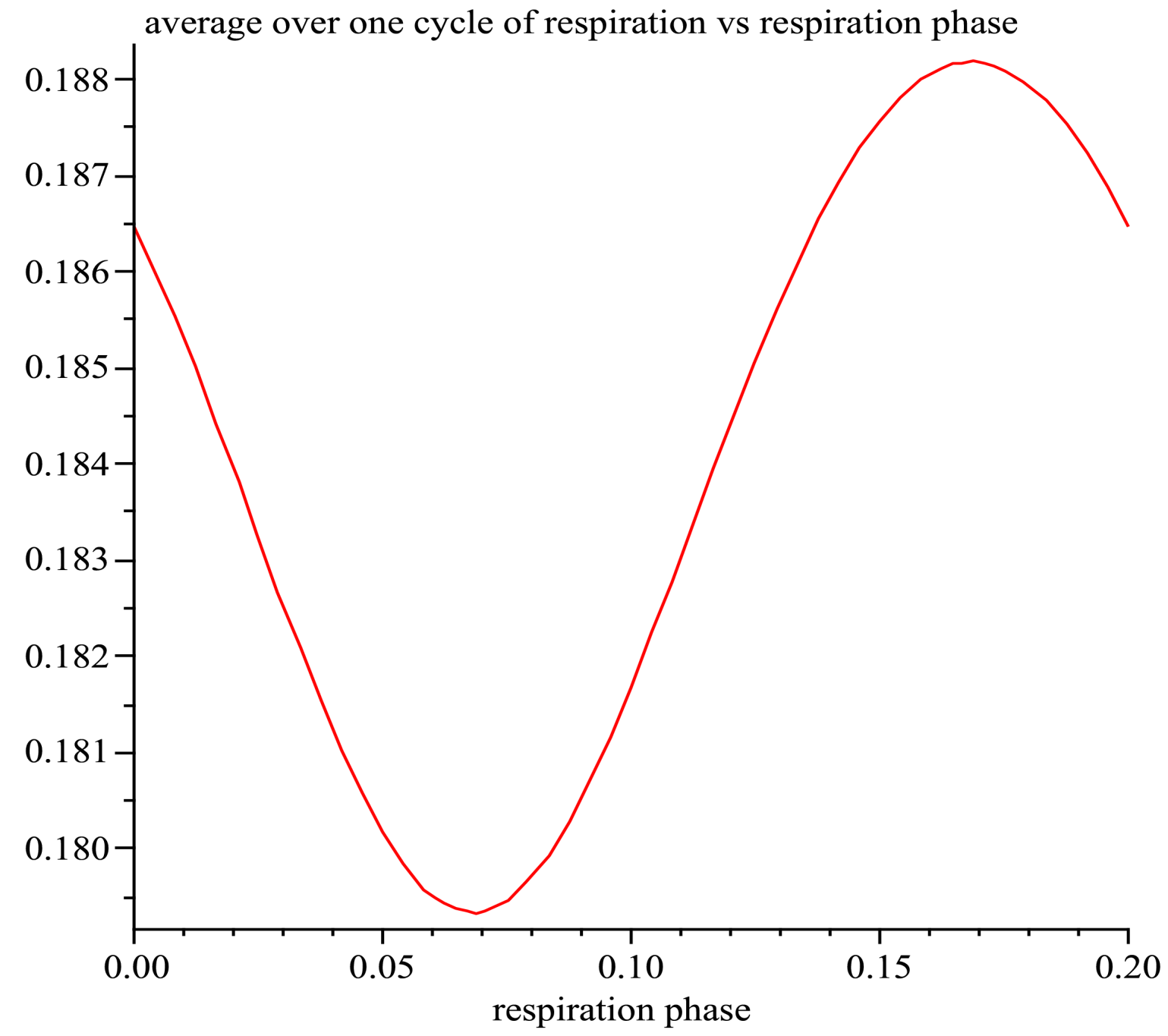

Figure 5.5: average value of the respiration influence vs respiration phase 
cycles, and approximating phase as identical to time:

$$
\begin{aligned}
f(t) F(t)=\frac{1}{3 \pi}\left(3 a_{0}\right. & +6 a_{1} \cos \left(\frac{\pi t}{a T}\right)+6 b_{1} \sin \left(\frac{\pi t}{a T}\right)-2 a_{0} \cos \left(\frac{\pi t}{T}+\phi_{d}\right) \\
& -2 a_{1} \cos \left(\frac{\left(\frac{1}{a}-1\right) \pi t}{T}-\phi_{d}\right)-2 b_{1} \sin \left(\frac{\left(\frac{1}{a}-1\right) \pi t}{T}-\phi_{d}\right), \\
& -2 a_{1} \cos \left(\frac{\left(\frac{1}{a}+1\right) \pi t}{T}+\phi_{d}\right)-2 b_{1} \sin \left(\frac{\left(\frac{1}{a}+1\right) \pi t}{T}+\phi_{d}\right) .
\end{aligned}
$$

There is a constant term, three fundamental terms, two sum terms, and two difference terms. Dynamically as the frequency of the heart oscillator changes under the effect of these quantities which are a function of the phases of the two oscillators, the phase difference $\phi_{d}$ between the heart interval and the respiration adjusts so that it balances the difference between the nominal frequency of the heart interval and the respiration as the heart interval changes to be rationally related to the respiration period.

What is the effect of the different terms in the Fourier series on the synchronisation? Approximately, if the variability in the coupling effect does not exceed $\pm \pi$, then a difference term will be able to synchronise the oscillators at a ratio $\frac{m}{n}$. The remaining oscillating terms of the coupling add to change the length of the heart interval depending on its relative place in the $m$ respiration cycles. This causes the deviation from the instantaneous synchronisation criterion of $\left|m \theta_{r}-n \phi_{h}\right|=$ const. But the average over the $m$ respiration cycles must be constant as the total phase over the synchronisation period must lead to $m$ ( $n$ heartcycles) = $n$ ( $m$ respirationcycles). Multiplying a system (5.1) by integers $m$ and $n$ respectively, does not change the frequency content of the coupling functions. So synchronisation at a particular ratio $\frac{m}{n}$ depends on the relative magnitudes of the terms.

If $m f_{1}=n f_{2}$ when there is synchronisation then $n T_{1}=m T_{2}$ where $T_{1}$ and $T_{2}$ are the periods of the oscillators. Then the synchronisation has a frequency of $m f_{1}=n f_{2}$ so Fourier components may exist at all integer multiples. Where there is no synchronisation then the signal is quasiperi- 
odic and the spectrum is continuous. This leads to the Fourier transform of the autocorrelation of the signal (the power spectrum) criteria for synchronisation where the autocorrelation spectrum shows peaks at intervals corresponding to the frequency of the synchronisation and the oscillator frequencies are rationally related. This must require a long time period to resolve the frequency components of the synchronisation. There must also be spectral peaks at the frequencies of the oscillators.

\subsection{RSA Synchronization detection}

Two methods to detect synchronisation of heart and respiration were used in numerical solutions to our model equations (4.1)-(4.13). The first method detects synchronisation through the phase difference of the oscillators. The phase difference of the respiration is tracked at each heart interval time. This corresponds to iterations of a map given by the Poincaré section through a torus. Synchronisation is found when there is a repeating sequence of phases. After the rotation number has stabilised the phase of respiration is set as a reference value, and the heart intervals calculated for 100 heart beats, while tracking the respiration phase to detect the reccurence of the reference value. Sequences which do not recur within 100 heart beats are not detected.

As the locking zones might be quite narrow for low values of coupling it is possible that zones might fall between discrete frequency steps as the respiration frequency is swept across a range. So to detect any synchronisation initially a quantified uncertainty is allowed in detecting the respiration reference phase. At heart beat $n$ the reference phase is detected to within $\pm \Delta$. So, for $m$ cycles of respiration the actual respiration frequency could be with equal probability, $f_{r} \pm f_{r} \cdot \frac{\Delta}{m}$ cycles/sec where $f_{r}$ is the nominal respiration frequency. So a frequency step of $2 \cdot f_{r} \cdot \frac{\Delta}{m}$ will detect all ratios up to $m$ respiration cycles. The uncertainty is a decreasing function of $m$ so synchronisation ratios with greater than $m$ respiration cycles will 
not be detected if they lie in between frequency steps. Synchronisation ratios with less than $m$ respiration cycles will be detected first.

Similarly the locking ratio uncertainty equivalent to the rotation number uncertainty is $\frac{m}{n} \pm \frac{\Delta}{n}$. Once the $m$ and $n$ values are determined they can be used to compare a calculated rotation number to $\frac{m}{n}$.

Plotting the rotation number against frequency for a respiration frequency range shows a Cantor set of locking zones with constant rotation number. Quasi-periodic behaviour is shown as a region of linear increase in rotation number against frequency. Noise processes may be present as well as other dynamical processes which will perturb the synchronising process. External dynamical processes driving the synchronisation can be exponential, or periodic, or quasiperiodic. A chaotic process can be treated as noise. If a driving process is exponential either it will decay away or synchronisation will be lost as the phase difference increases continually with the oscillators slipping cycles. Therefore the state variable of the phase difference might have a periodic component or noise component which is averaged over time.

A program was written to detect the synchronisation of respiration with heart interval using the Matlab model. As the synchronisation ratios present are initially unknown these have to be detected first. To do this the respiration frequency is stepped with a coarse $\Delta$ across a frequency range of interest and a synchronisation ratio determined for each frequency step. A number of heart intervals are allowed as a transient response to the respiration frequency step. At the end of each heart interval the phase of the heart oscillator is zero so the phase difference between the respiration and the heart is given by the respiration phase. At a heart interval time the respiration phase is observed to be a reference value and then if synchronisation is present this phase will re-occur $n$ heart intervals and $m$ respiration cycles later. The reference phase value is not affected by the higher order product terms but the intermediate values of phase do not satisfy the $|m \phi-n \theta|=$ const criterion for synchronisation but oscillate back and 
forth around the nominal value given by the criterion. So to determine the existence of synchronisation the reference respiration phase value is updated each time it is detected to establish a mean value. This also allows for residual transient response and any noise. The interference of noise and nonautonomous dynamics means that in some situations detection would need to be based on stochastic methods.

In the current study it was assumed no nonautonomous signals were present. An uncertainty of $\Delta$ in the phase detection is allowed for initally and detection is then refined. This will mean that a significant ratio will be detected anyway even if there is not any entrainment of the heart interval after a sufficient number of heart intervals. This establishes a limit on the number $n=\frac{1}{\Delta}$ of heart intervals it is useful to count up to.

This establishes a locking ratio and the respiration phase at this ratio to subsequent heart intervals is averaged over a number of cycles to confirm the locking ratio and find the mean reference phase. Having found important synchronisation ratios at a range of respiration frequencies the exact extent of each locking zone is then established by searching across two frequency steps for the zone boundaries using an interval bisection of the frequency interval.

The results of running the heart model showed that the effect of the uncertainty of $\Delta$ in the initial detection did result in a large number of narrow apparent locking zones but as the initial results were refined practically all the locking zones disappeared except for 2 or 3 of the significant zones. This was a consequence of the locking regions being much less significant than anticipated. Therefore it was found to be more practical to plot the rotation number over a small range of respiration frequency where the ratio would be present.

A second method used the autocorrelation of the power spectrum of the heart interval times to indicate any synchronisation graphically. The autocorrelation has the effect of integrating harmonically related components. The Fourier components of the oscillator signal which are integer 
multiples of the time frame are meaningful. Clearly using a time frame $T$ which contains an integral number of heart oscillator intervals $n$ is advantageous as this identifies the Fourier components with harmonics of the heart interval. In calculating an ensemble average it is necessary to use the same length of time $T$ for each frame of signal. This means that the number of heart intervals is not exactly integral due to the slight variance from the average heart interval length of a finite number $n$ of heart intervals. For the synchronisation ratio, as it would be unknown the multiple of synchronisation periods would often not be related to the time frame $T$. This results in a band of $\frac{n}{T}$ Fourier frequency components around the sychronisation frequency, because the spectrum has the form of $\frac{\sin x}{x}$ with zeros at a spacing of $\frac{1}{T}$ relative to the centre frequency, with the centre frequency at the frequency of synchronisation.

Calculating the Fourier components of a sine wave nonsynchronous with the time interval it is seen there are sum and difference frequency terms to integrate. i.e. $\cos \left(\left(\omega_{m}-\frac{2 \pi n}{T}\right) t\right)+\cos \left(\left(\omega_{m}+\frac{2 \pi n}{T}\right) t\right)$. The sum term contributes little as it varies rapidly. It is the the fractional part of the sine wave in the interval $T$ which contributes to the amplitude of the component, as the whole cycles average to zero. For a rapidly varying sine wave this must be for a correspondingly shorter part of the integration time. The difference term therefore has a fractional period of the sine wave contributing for a greater part of the integration over the interval $T$ depending on the closeness of the nonsynchronous sinewave to a $\frac{n}{T}$ Fourier component. It might be assumed that the time $T$ is sufficiently long so that many cycles of heart interval are included and that the fractional interval length is relatively small compared to $T$ so that the average frequency of the heart intervals is close to a Fourier component $\frac{n}{T}$. This is also true for the slower oscillator of the synchronising pair, in this case the respiration. For an $\frac{m}{n}$ ratio the period of the synchronisation is $m T_{r}=n T_{h}$ and this could be a significant fraction of $T$. It is this period that is fundamental in the frequency spectrum and multiples of this frequency appear at the Fourier 
components $\frac{n}{T}$.

For quasiperiodic behaviour there is no periodicity in the time frame $T$ and $\frac{1}{T}$ is the fundamental component. So qualitatively the spectrum consists of components at a spacing of $\frac{1}{T}$, which depend in amplitude on how synchronous the waveform is in the interval $T$, that is how many cycles of synchronisation occur within the time interval $T$ or none at all for quasiperiodic waveforms. At multiples of the frequency of the synchronisation $\left(m T_{r}=n T_{h}\right)$, there is a peak at $\frac{n}{n T_{h}}$ which is the average frequency of the heart interval, and the respiration components are at integer multiples of $\frac{m}{m T_{r}}$. The effect of quasiperiodic behaviour is that $m, n$ change suddenly from being relatively small to relatively large or infinite as the quasi-periodicity is indistingushable from a period of synchronisation larger than $T$, and the periodicity at the synchronisation frequency in the signal is suddenly lost. The period of the signal is now $T$ so the synchronising effect on the heart intervals of the respiration now appears in all $\frac{1}{T}$ components. The components of $\frac{1}{T}$ are more spread out around the multiples of the two oscillator frequencies, giving an autocorrelation power spectrum that is broadened around its main peak at $\frac{1}{T_{h}}$ also. This is apparent in the baseband where the single component at the synchronisation frequency when the respiration and heart interval are synchronous becomes many $\frac{1}{T}$ components when the respiration is not synchronous. In a nonlinear system where there are Arnold tongues the oscillators remain locked and synchronised as the respiration frequency changes, so the magnitude of the $\frac{1}{T}$ components also remains fixed, until the boundary of the Arnold tongue is crossed when there is a sudden jump in the magnitude of the $\frac{1}{T}$ components, as the rotation number also changes with a step.

The figures (5.6)-(5.9) show the result of power frequency spectrum analysis of heart interval data generated by the Matlab model. They do bear out the reasoning of fundamental Fourier components appearing for quasiperiodic behaviour. The graphs are calculated from a time interval $T$ of 1024 times the average heart interval length for 33 x 1024 heart 
heart interval power spectrum

rot $\mathrm{no}=5 \mathrm{fstep}=0.0010283 \mathrm{~Hz}, \mathrm{kprsa}=0.2, \mathrm{ksrsa}=0.2$

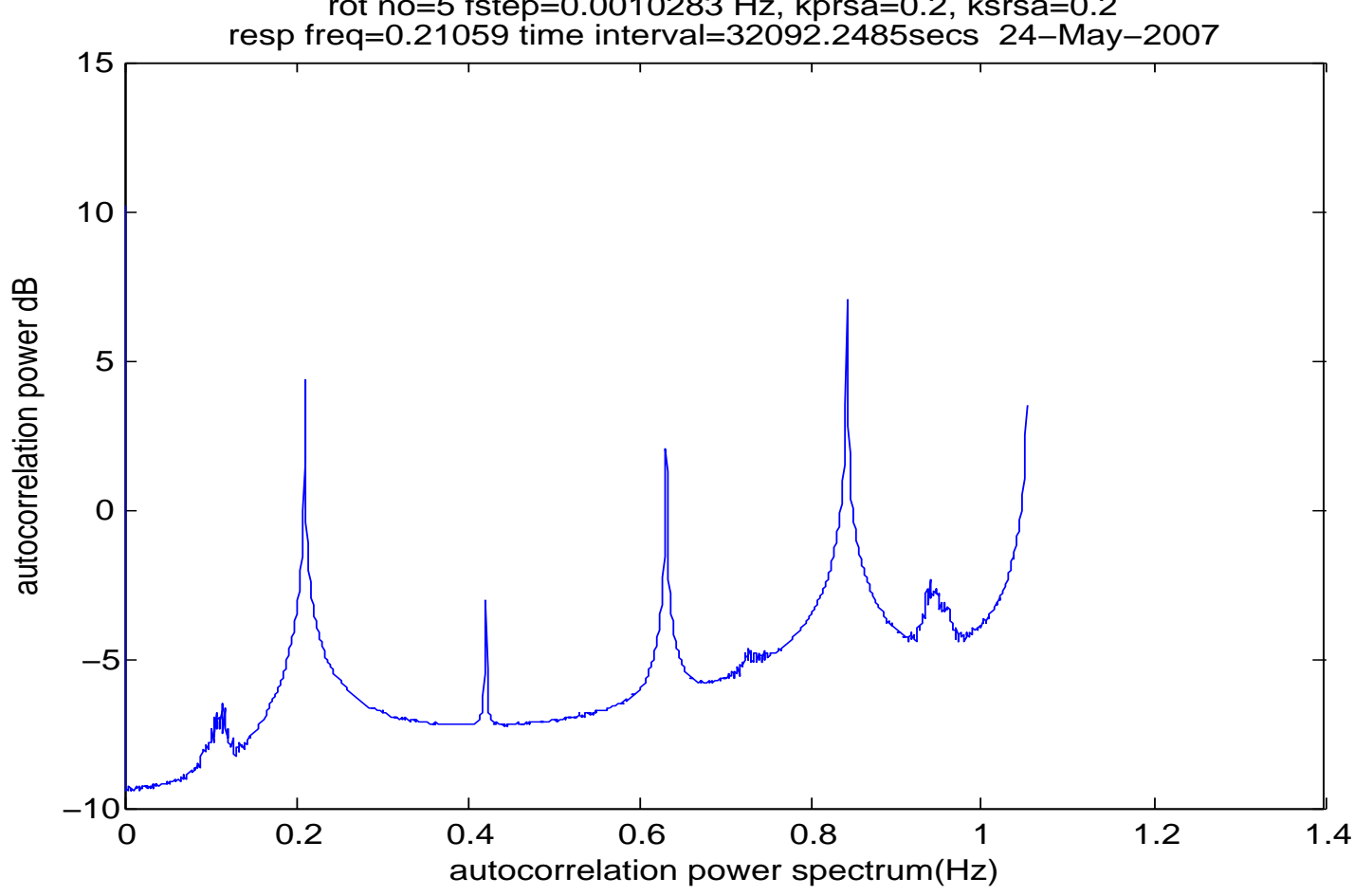

Figure 5.6: autocorrelation power for 5:1 ratio 
heart interval power spectrum

rot no $=5.0001$ fstep $=0.0010281 \mathrm{~Hz}, \mathrm{kprsa}=0.2, \mathrm{ksrsa}=0.2$

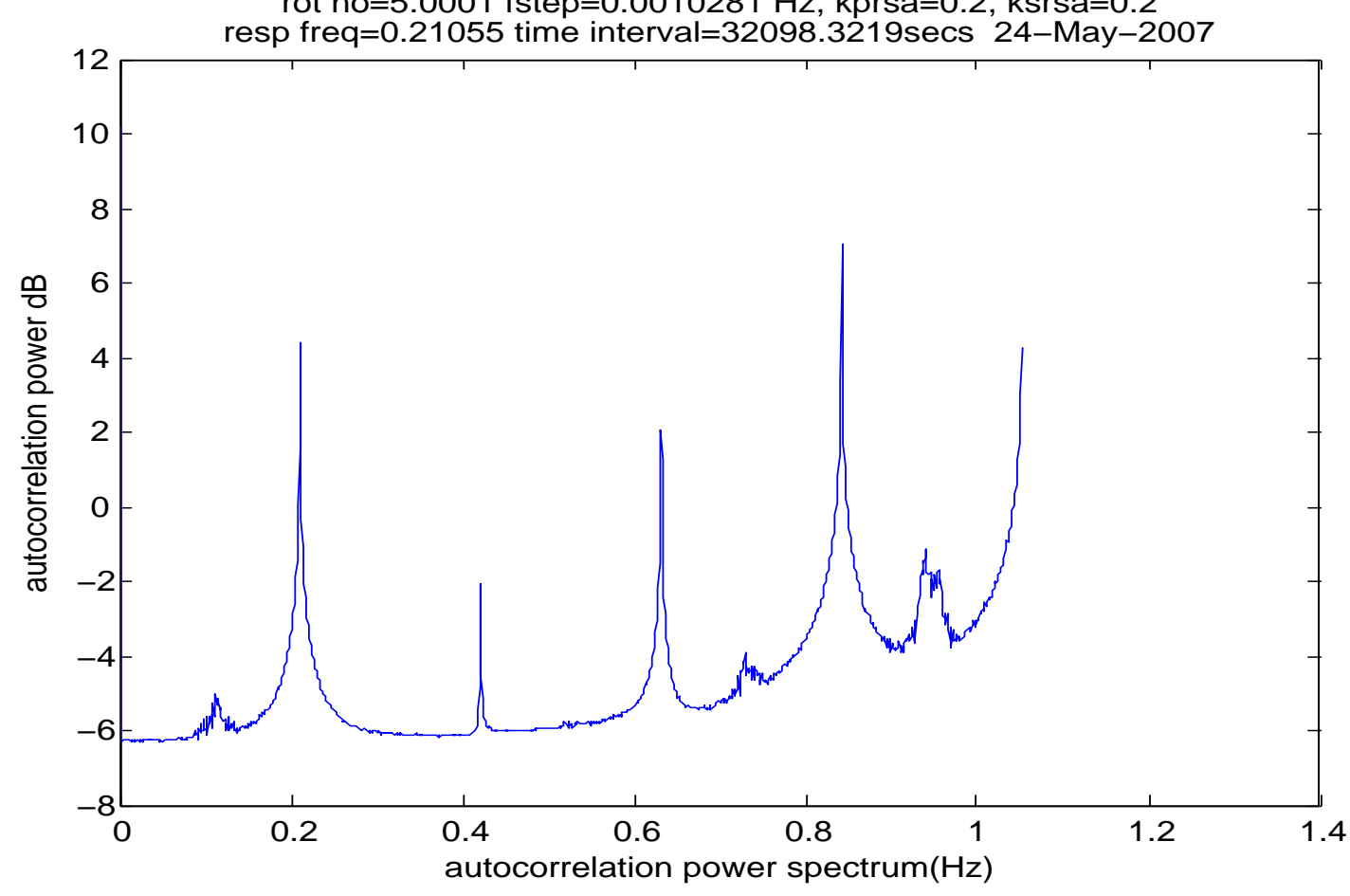

Figure 5.7: a rotation number slightly greater than 5:1 
heart interval power spectrum

rot no $=5.269$ fstep $=0.0010291 \mathrm{~Hz}$, kprsa $=0.2$, ksrsa $=0.2$

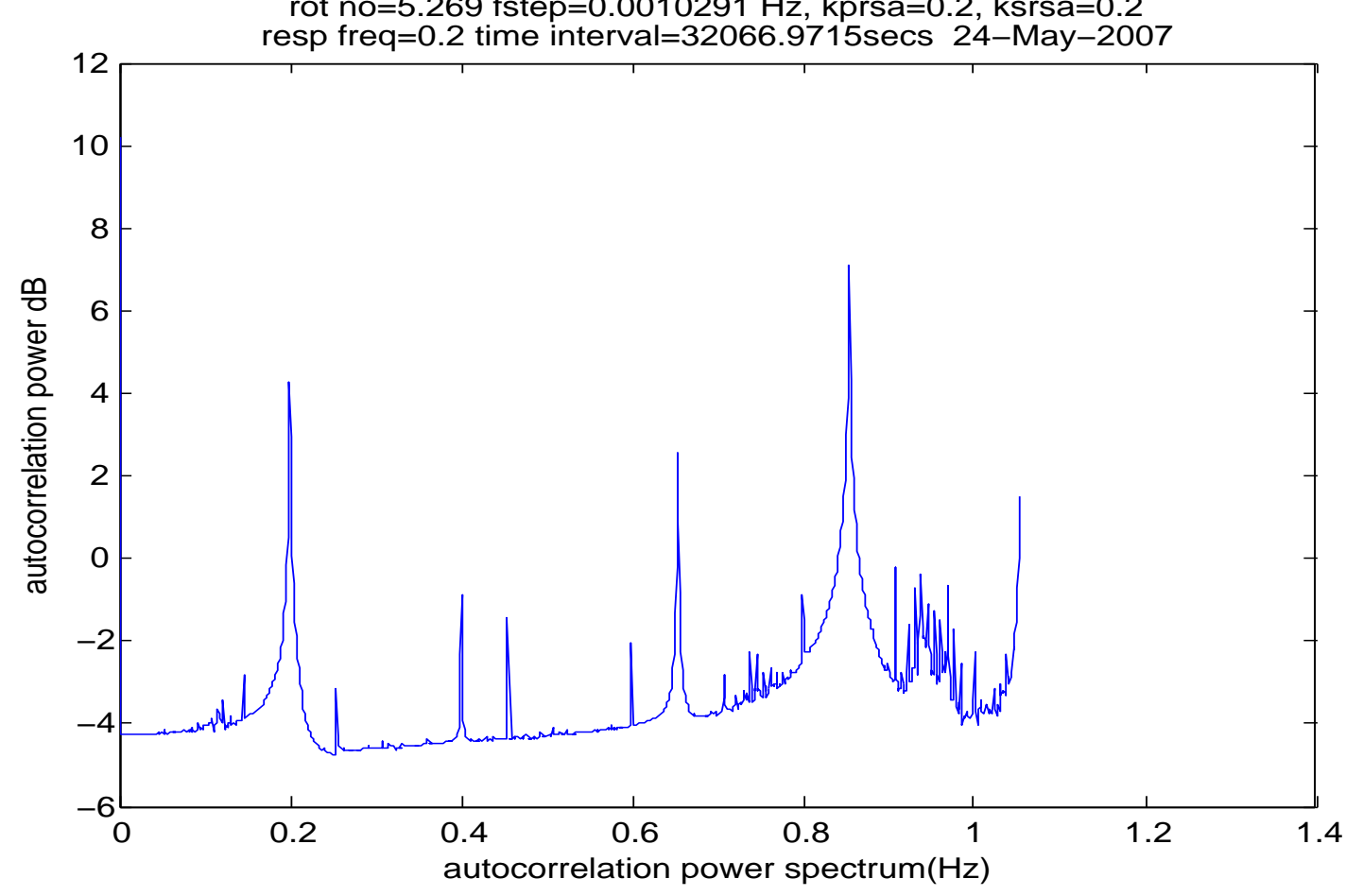

Figure 5.8: quasiperiodic behaviour 
heart interval power spectrum

rot no $=4.5$ fstep $=0.001031 \mathrm{~Hz}, \mathrm{kprsa}=0.2, \mathrm{ksrsa}=0.2$

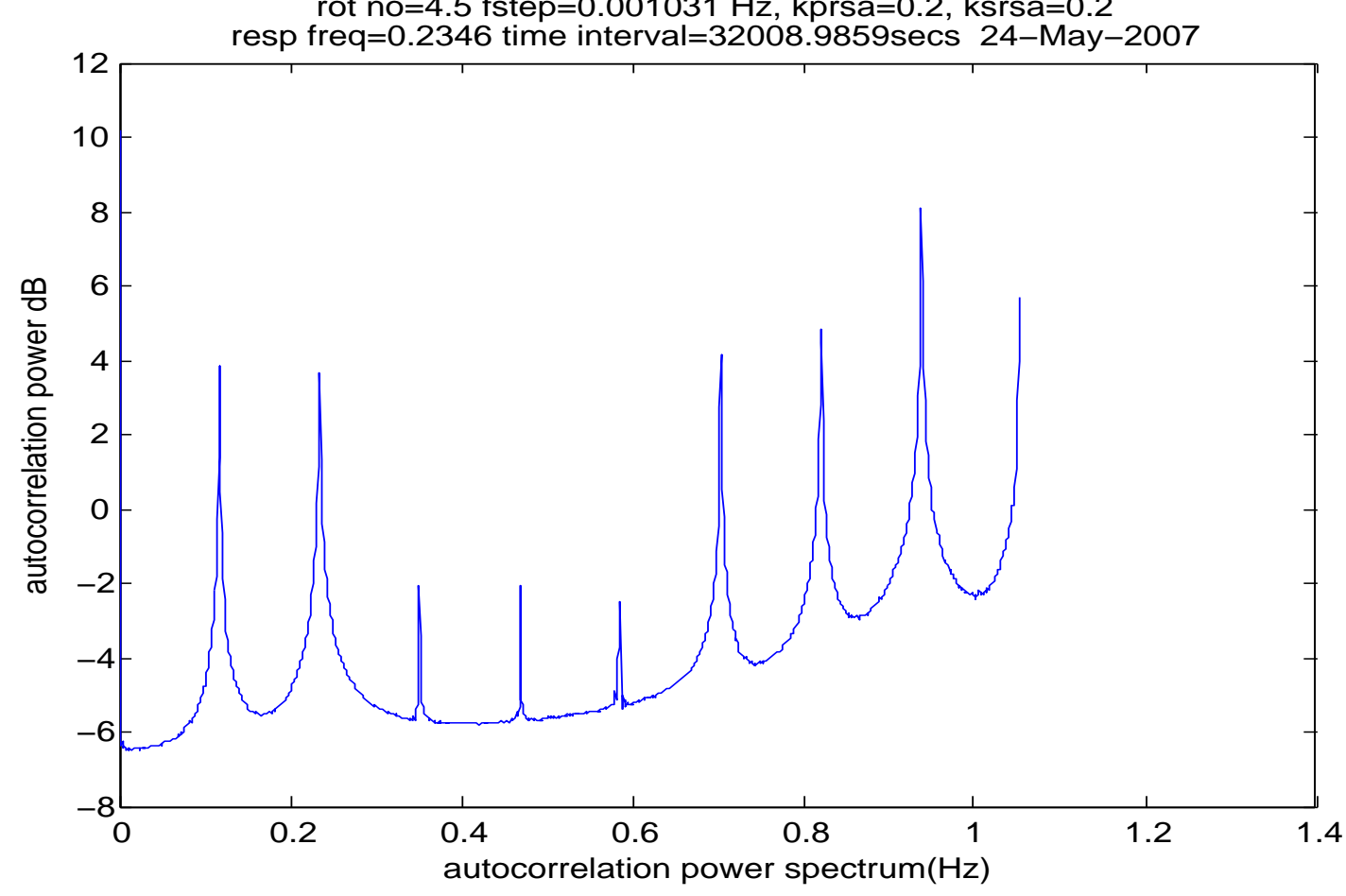

Figure 5.9: locking ratio 9:2 
intervals. The total time length of the data is printed on the graphs. This gives an ensemble of about 32 intervals over which the frequency data is averaged. 1024 frequency points are taken which means that the highest frequency is 1 step less than the average heart interval frequency, the carrier component. The average interval frequency for each time interval $T$ must vary statistically, according to the heart interval statistics.

Figures (5.6) and (5.7) show a locking ratio of 5:1 and just outside this ratio with a rotation number of 5.0001 respectively. The most distinctive feature is the increase in fundamental $\frac{1}{T}$ components for figure (5.7). The level increases by about $3 \mathrm{~dB}$ for a .0001 change in the rotation number. Another feature, not expected, are low levels of narrow band noise around $2 x$ the respiration frequency. These are also sensitive to the locking zone. These are not readily explicable and seem to relate to dynamical processes occuring perhaps in the context of a system resonance. It is noted that although the RSA coupling coefficient is $2 x$ the nominal physiological value, coupling is relatively weak and the rotation numbers still a little unstable at this coupling, as seen in section (5.5). The fundamental respiration components, also the synchronisation frequency at a 5:1 locking ratio, are apparent at slightly greater than $.2 \mathrm{~Hz}$ as the heart interval frequency is a little more than $1 \mathrm{~Hz}$. Figure (5.8) clearly shows quasiperiodic behaviour. The level of the fundamental $\frac{1}{T}$ components is elevated throughout the spectrum. Other components result from the spectrums of the carrier and the respiration modulation no longer being integral multiples. Figure (5.9) shows the 9:2 ratio. In this case the fundamental $\frac{1}{T}$ components are at a lower level as expected and also there exist $\frac{1}{2} \mathrm{x}$ respiration sub harmonic frequency components at the synchronisation frequency. 


\subsection{RSA locking regions of the Cardiovascular system}

The coupling of the respiration to the heart oscillator through RSA was studied to find its significance in the cardiovasular system. To detect locking zones the respiration frequency was swept across a respiration frequency range containing a significant synchronisation ratio. The RSA coupling has proved to be quite weak so only a narrow range of frequencies was needed to show an entire locking region.

The plots were made for a number of significant ratios with normal physiological parameters and a range of coupling coefficients for the parasympathetic and sympathetic loops. The effect of the RSA coupling is seen to be quite weak. The response of the peripheral resistance to pressure changes and the contractility variation significantly degrade the locking regions as it was found that the locking regions were an order of magnitude greater with constant peripheral resistance and contractility. At a normal coupling coefficient $k_{r s a}=.1$ it can be seen that the locking region is not clearly defined. Figure 5.10 shows the variation in rotation number as the number of heart intervals increases. This is caused by the heart interval changing within the respiration cycle. Without synchronisation of the heart interval and the respiration a number of heart intervals are required to determine the rotation number. The variance of the respiration phase at each heart interval add, as it is assumed not to be correlated, so the rotation number is converging at a rate $\frac{1}{\sqrt{N}}$. This is the case for noise or if the frequencies are incommensurate. If the frequencies are synchronised then the harmonically related jitter is also periodic at $q$ respiration cycles for a $\frac{p}{q}$ synchronisation and the rotation number is precise at each $q$ cycles of respiration. 


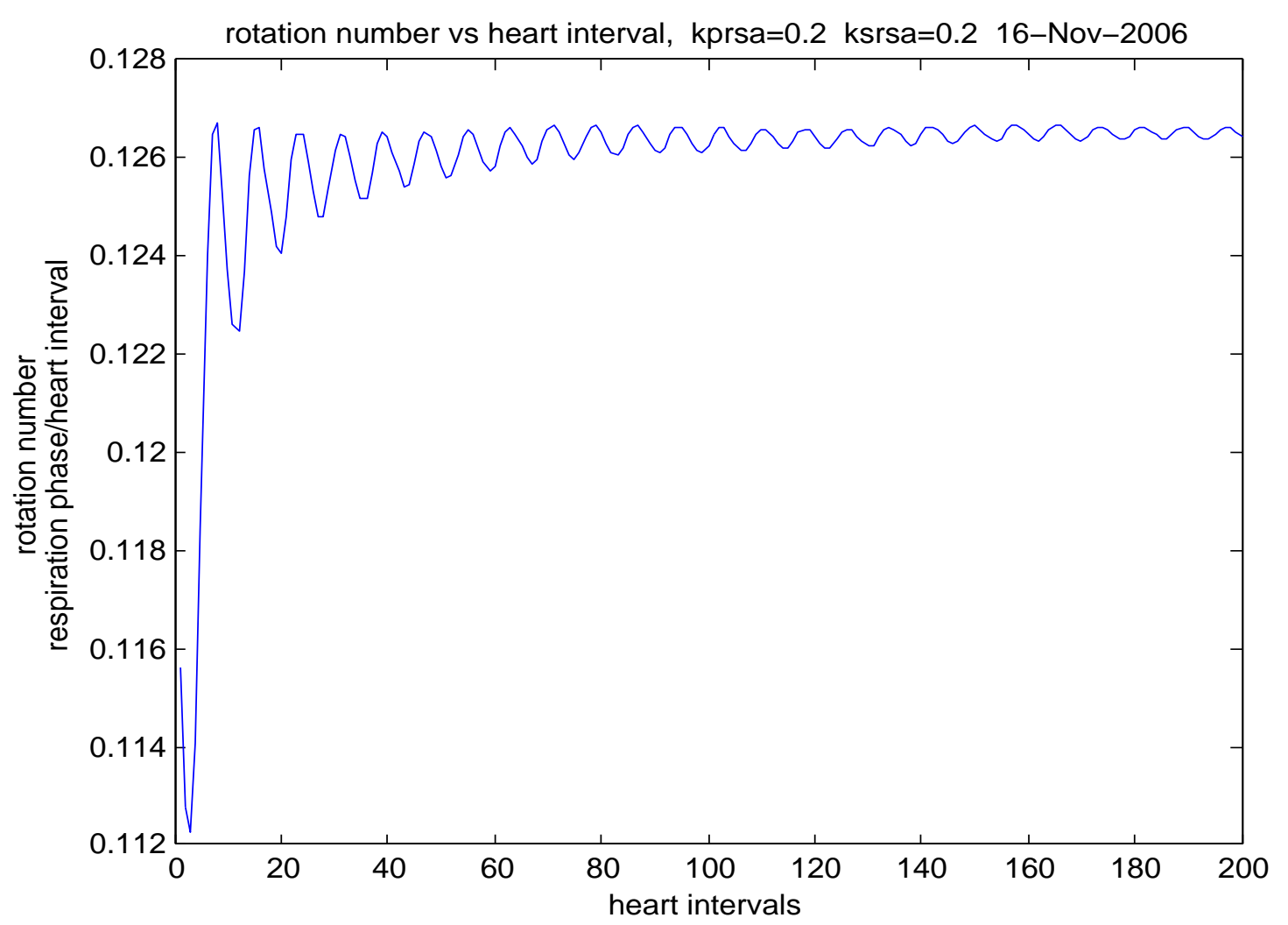

Figure 5.10: convergence of rotation number 


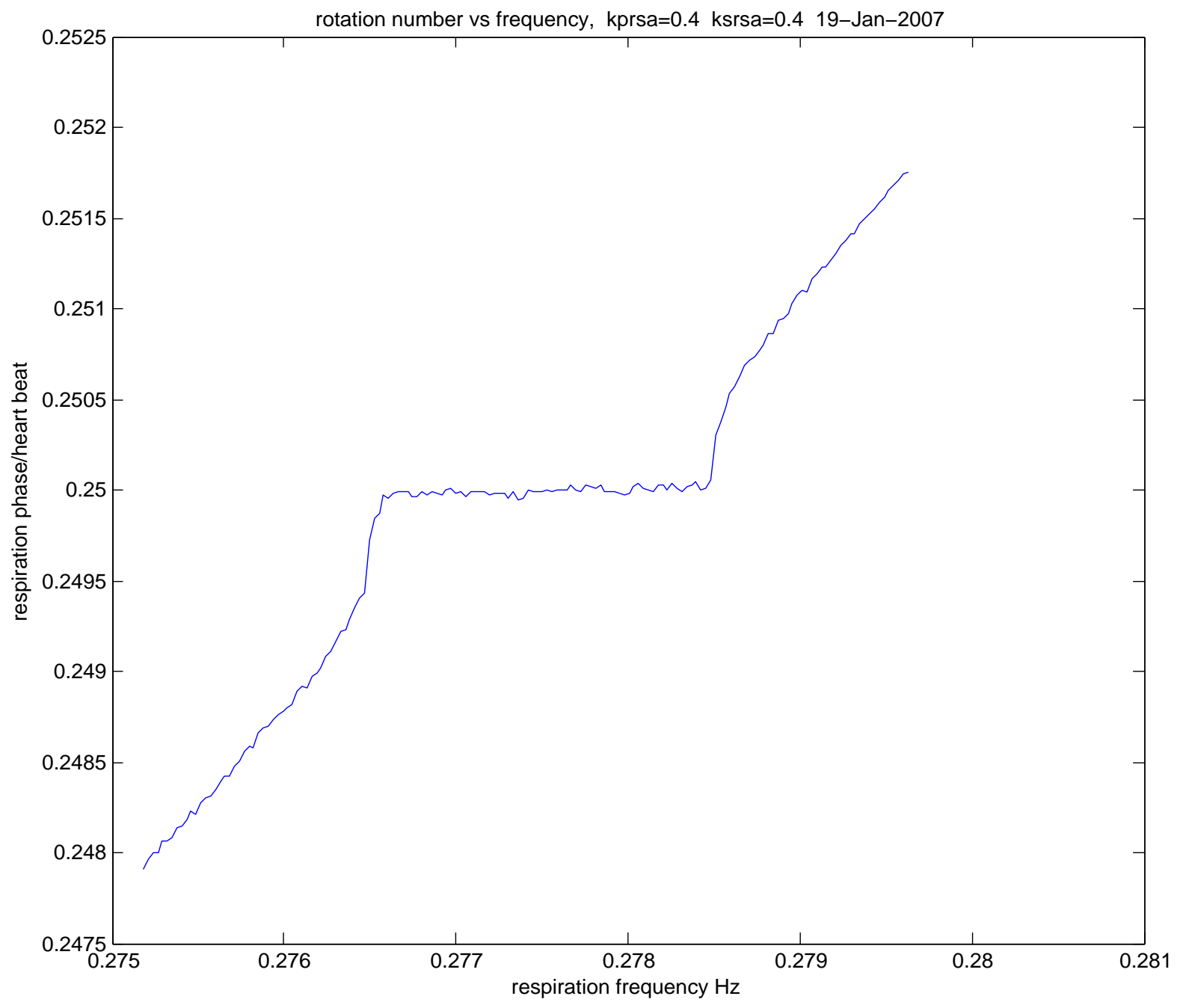

Figure 5.11: rotation number plot for 4:1 region 


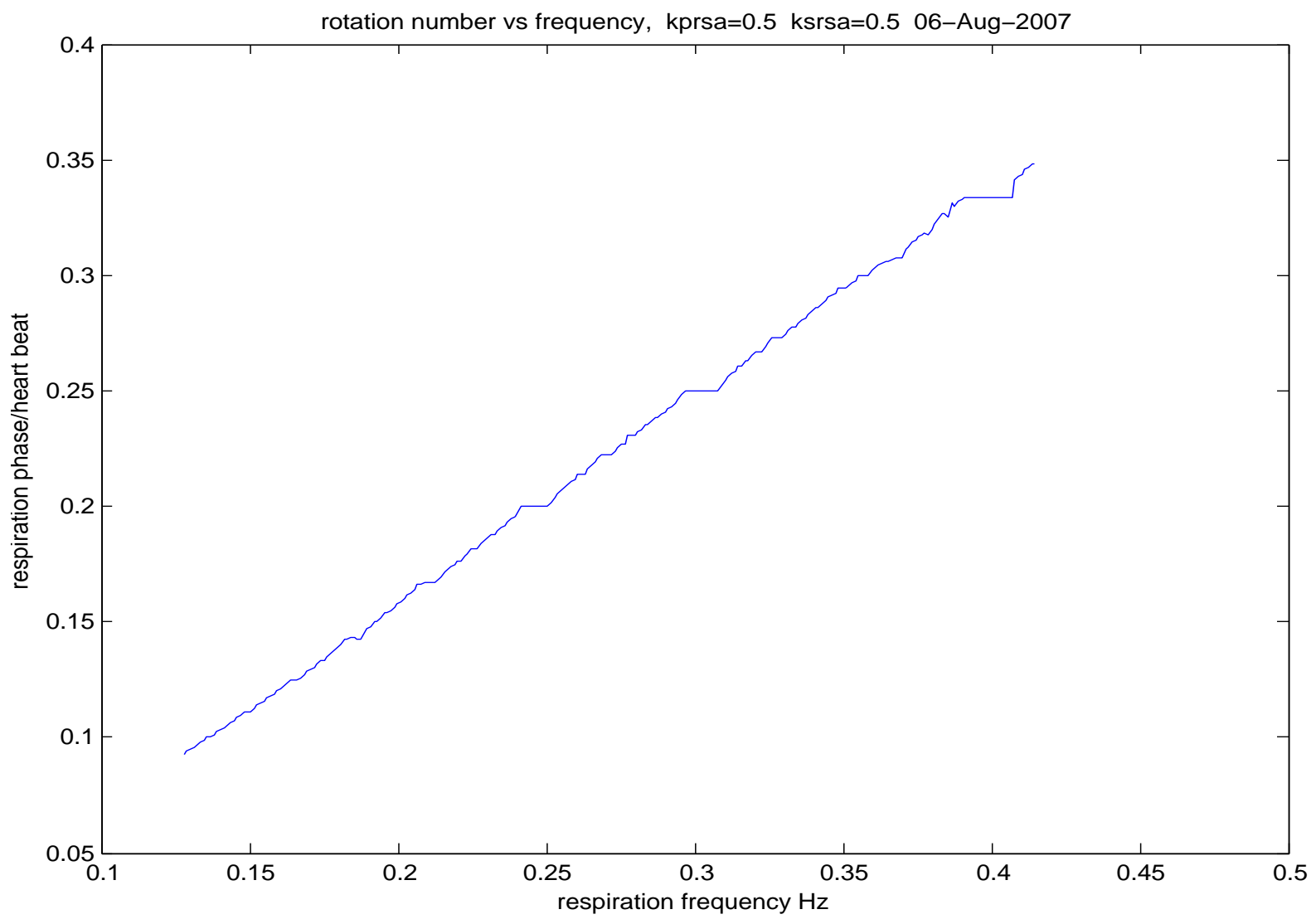

Figure 5.12: range of respiration frequencies showing locking regions from 3:1 


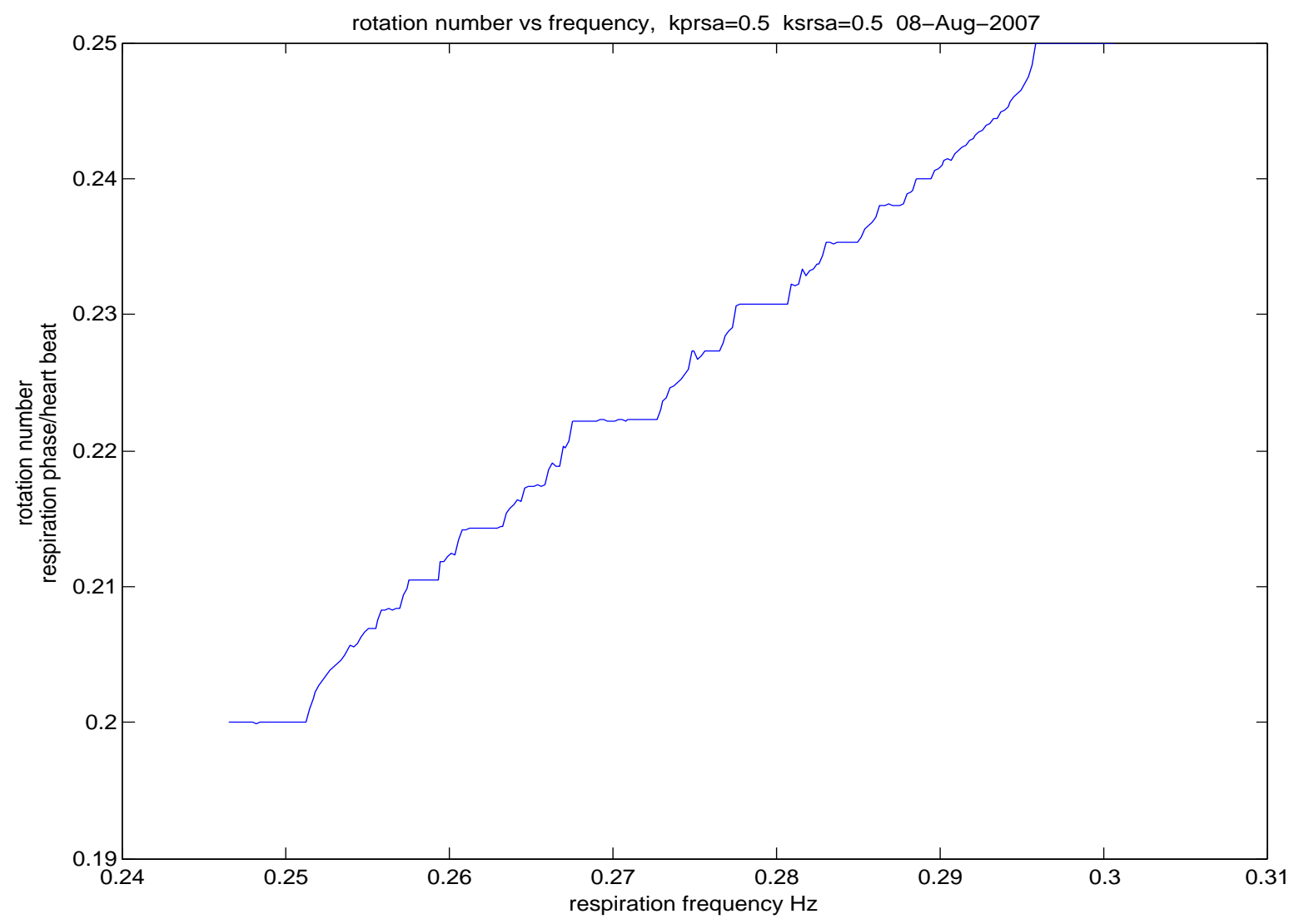

Figure 5.13: detail of locking regions between 5:1 and 4:1 


\begin{tabular}{|l|l|l|l|l|l|l|}
\hline & & ratio & & & & \\
\hline & & $3: 1$ & $4: 1$ & $5: 1$ & $6: 1$ & $7: 1$ \\
\hline RSA & .1 & .0009 & .0003 & .0002 & .0001 & .00005 \\
\hline & .2 & .0016 & .0006 & .00025 & .0002 & .0001 \\
\hline & .3 & .0019 & .0006 & .0005 & .0004 & .0003 \\
\hline & .4 & .0034 & .00185 & .0016 & .0016 & .0008 \\
\hline & .5 & .0204 & .0127 & .0100 & .0069 & .0058 \\
\hline
\end{tabular}

Table 5.1: Locking zone width, in respiration frequency $(\mathrm{Hz})$

\begin{tabular}{|l|l|l|l|l|l|l|}
\hline & ratio & $3: 1$ & $4: 1$ & $5: 1$ & $6: 1$ & $7: 1$ \\
\hline RSA & .1 & $.3428-.3437$ & $.2573-.2577$ & $.20492-.20615$ & $.1718-.1719$ & $.1476-.1476$ \\
\hline & .2 & $.3496-.3512$ & $.2627-.2633$ & $.2105-.21075$ & $.1759-.1761$ & $.1521-.1522$ \\
\hline & .3 & $.3574-.3593$ & $.2687-.2693$ & $.2156-.2161$ & $.1818-.1822$ & $.1588-.1591$ \\
\hline & .4 & $.3664-.3698$ & $.2766-.2785$ & $.2435-.2451$ & $.1905-.1921$ & $.1674-.1682$ \\
\hline & .5 & $.3868-.4072$ & $.2957-.3084$ & $.2413-.2513$ & $.2063-.2132$ & $.1817-.1875$ \\
\hline
\end{tabular}

Table 5.2: Locking zone boundaries, in respiration frequency $(\mathrm{Hz})$

The law $k \cdot \varepsilon^{x}$ is used to explain the locking region widths where $k$ is a constant of proportionality and $\varepsilon$ is the coupling coefficient $\left(=k_{r s a}\right)$. Following are tables of locking ratio data obtained from numerical simulation.

It is seen that the nominal RSA coupling coefficient must have a constant of proportionality to reflect the actual coupling, so this must be included in a power law rule, $a \cdot(c \cdot \varepsilon)^{x}$ so the constant of proportionality must also be subject to the power law. The locking zone width is related to the Farey sequence that the ratio first occurs in and this is given by the denominator of the locking ratio, so $x=n$ where $n$ is the denominator of the Farey sequence.

One problem is that due to the frequency response of the system the actual coupling is higher at higher respiration frequencies. This is due to 
the respiration frequency becoming comparable to the sympathetic time delay so the peripheral resistance no longer counteracts the RSA. A resonance effect could be envisaged. The lower ratios will have higher coupling than indicated by the nominal value. This is shown by the data of the 3:1 ratio as the locking zones increase dramatically at this ratio. A second departure from the norm is a nonlinear effect which becomes apparent at a coupling coefficient of $>.4$. In this case the RSA coupling is dominating the baroreflex feedback and the heart oscillator is being driven in an open loop fashion.

Using Maple the data was first fitted to $k \cdot \varepsilon^{n}$ for each locking zone 3-7 using the .1-.4 range of coupling coefficients.

\begin{tabular}{|c|c|c|c|c|c|}
\hline \multicolumn{6}{|c|}{ Coefficient of linear fit to $\varepsilon^{n}$} \\
\hline $\mathrm{n}$ & 3 & 4 & 5 & 6 & 7 \\
\hline $\mathrm{k}$ & .05779 & .07354 & 15948 & .39613 & .50419 \\
\hline
\end{tabular}

The residual mean square is of order $10^{-8}$ in each case. This data is fitted to the law $a \cdot b^{n}$ where $n$ is the rotation number. This gives $a=.013783$ and $b=1.6856$, with the residual mean square $=.0032$.

The exercise is repeated without the $3: 1$ ratio or the .4 coupling, with the following results.

\begin{tabular}{|l|l|l|l|l|}
\multicolumn{5}{c}{ Coefficient of linear fit to $\varepsilon^{n}$} \\
\hline $\mathrm{n}$ & 4 & 5 & 6 & 7 \\
\hline $\mathrm{k}$ & .08580 & .21590 & .56859 & 1.39383 \\
\hline
\end{tabular}

The residual mean square is approximately the same, of order $10^{-8}$. Fitting to the law $a \cdot b^{n}$ gives $a=.0023274$ and $b=2.4937$, with the residual mean square $=.0000866$. So the nonlinear and frequency response effects are affecting the Arnold tongues considerably. 


\subsection{Dynamics of RSA phase locking}

\subsubsection{Step response of heart system}

A step function was used as input for the RSA of the complete heart model. Rather than coupling a half sine wave to model the influence of respiration a Heaviside function was used. The system variables were recorded before and after the step. Heart interval length, diastolic pressure, and sympathetic transmitter concentration are plotted in figures 5.14-5.16.

This shows the effect of the RSA and the parasympathetic loop and sympathetic loops on the response of the heart oscillator. Inspection of the model equations (4.1)-(4.7) shows that the sympathetic RSA coupling counteracts the parasympathetic RSA coupling due to the RSA being modelled as centrally coupled, although the parasympathetic and sympathetic loops have the same effect in controlling heart interval length. In figure 5.14 it is seen that the sympathetic RSA coupling causes the heart interval time to decrease while the parasympathetic RSA coupling is causing it to increase. This shows the much higher gain of the sympathetic loop is controlling the heart interval time. At the same time the effect of the sympathetic baroreflex loop and the sympathetic control of the peripheral resistance is to counteract any changes in diastolic pressure. The peripheral resistance has the greatest overall effect but a small diastolic pressure change from the nominal value is still required to counteract the RSA. So although the initial diastolic pressure change is a large step due to the parasympathetic coupling it rebounds in the opposite direction due to the action of the sympathetic loops.

The ringing is a consequence of the high gains in the sympathetic loops and underdamping as the sharp initial step takes some time to decay away. It is seen that the heart oscillator is nonisochronous, as the heart interval length varies with the diastolic pressure, which is closely related to the sympathetic transmitter levels and so can be taken to indicate these levels. As a second order system it is underdamped as shown by the ringing 
which has the effect of slowing the return of the heart interval length to the limit cycle. The two Lyapunov exponents of such a system must be negative and relatively small. The ringing corresponds to a rotation in the phase space which the Lyapunov exponents are not able to show.

\subsubsection{Phase relationship across locking region}

Figures 5.17-5.19 show a plot of the heart interval time in terms of respiration phase and across a range of respiration frequencies. The horizontal axis is respiration phase, the vertical axis respiration frequency, and the depth is diastolic pressure. The respiration phase is plotted for about 50 heart intervals at a respiration frequency about a $5: 1$ locking region. It can be seen where the heart oscillator is synchronised to the respiration the points map onto each other and there are 5 points along the respiration phase axis. The spacing between the points varies with the respiration phase rather than being equally spaced, due to the direct response of the heart oscillator to the respiratory influence. Across the respiration frequency the points change in phase as the phase difference required to hold the heart oscillator in synchronism changes. Where the oscillators are not synchronised the points trail in a line as the phase of the oscillators slip with respect to the other. More points would give a better illustration of this. The diastolic pressure is thought to be a variable that expresses the most about the state of the heart system after the heart interval as it has the most effect on the sympathetic transmitter concentrations.

\subsubsection{Maps of the cardiovascular system}

Figure 5.20 shows the effect of the RSA on the return time of the heart interval. The $t_{n+1}$ time depends on whether $t_{n}$ is on the rising or falling slope of the respiration. A 2 dimensional embedding was also plotted in $3 \mathrm{D}$ but this revealed no new features. 


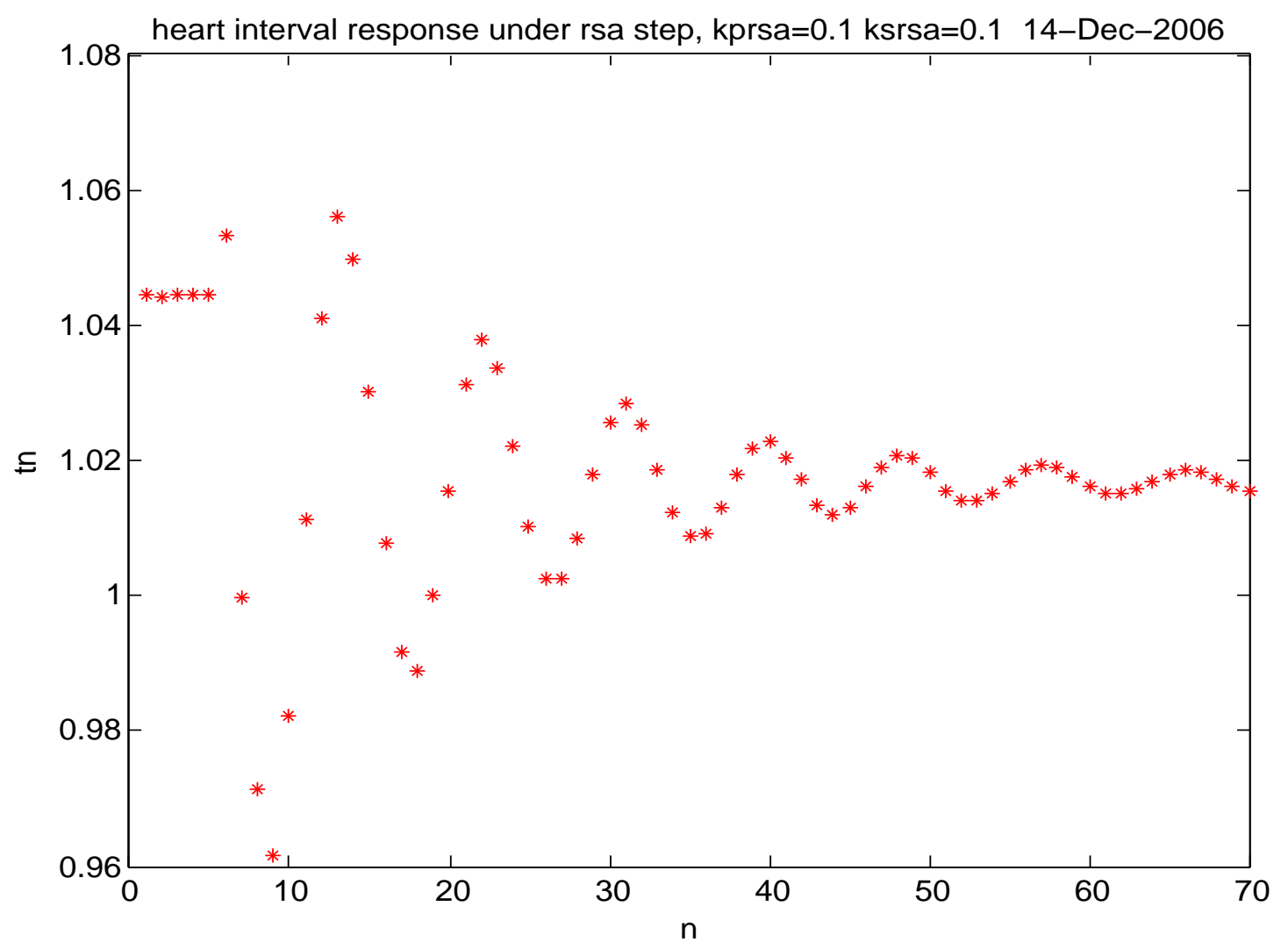

Figure 5.14: heart interval step response to RSA 


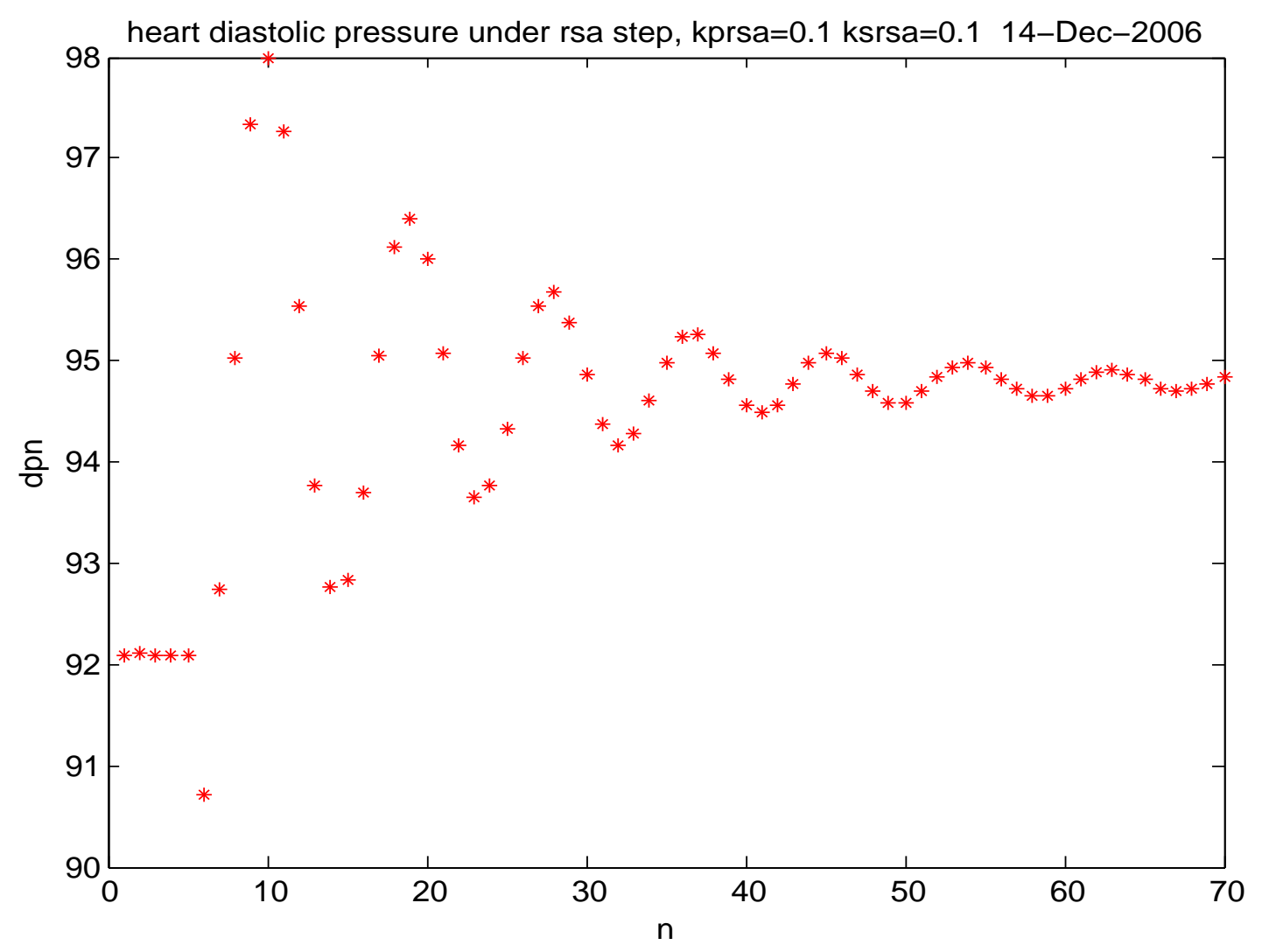

Figure 5.15: diastolic pressure step response to RSA 


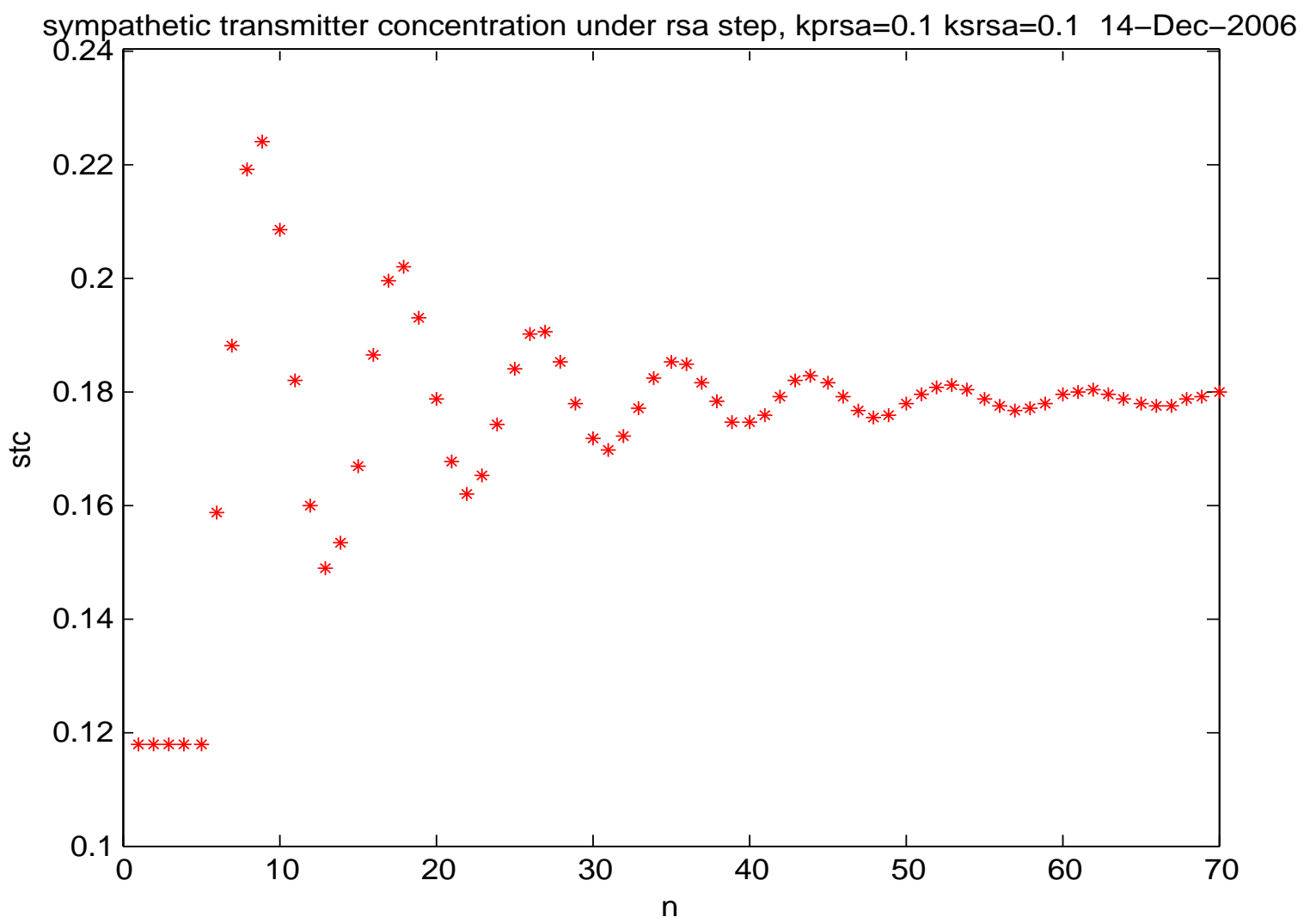

Figure 5.16: sympathetic transmitter step response to RSA 


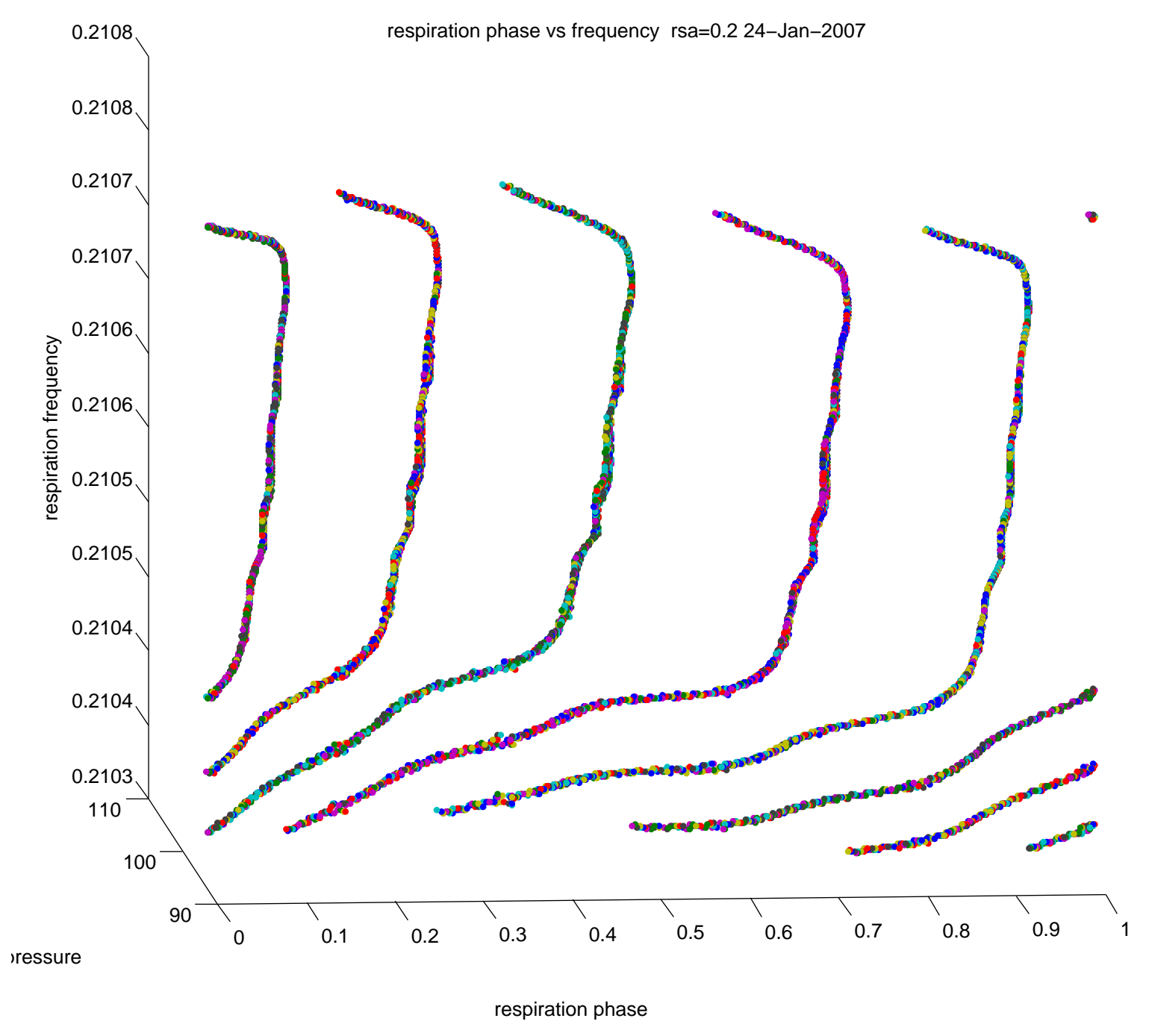

Figure 5.17: A view of a 3d plot of respiration frequency vs heart interval in terms of respiration phase vs diastolic pressure 


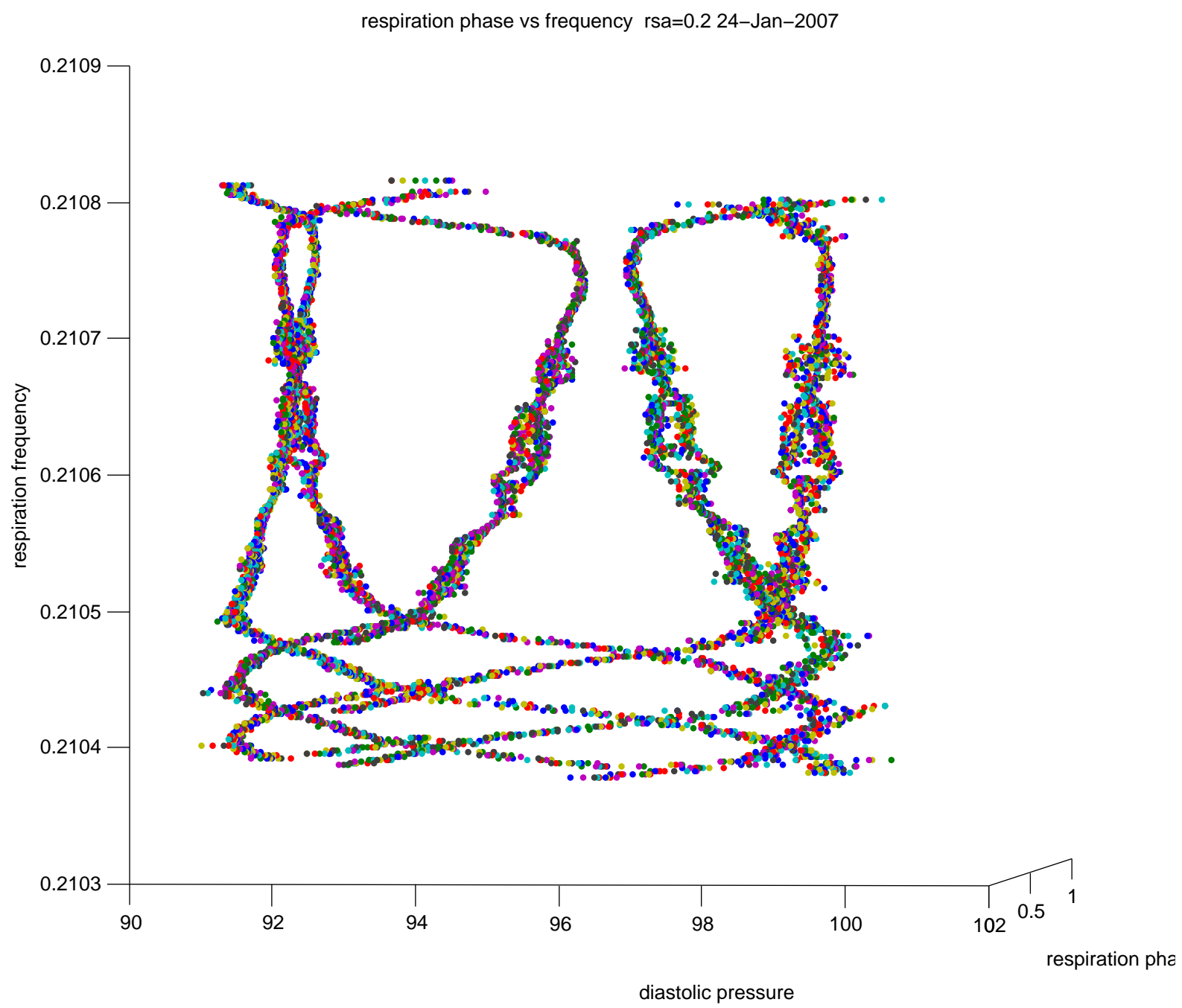

Figure 5.18: view from diastolic pressure axis 


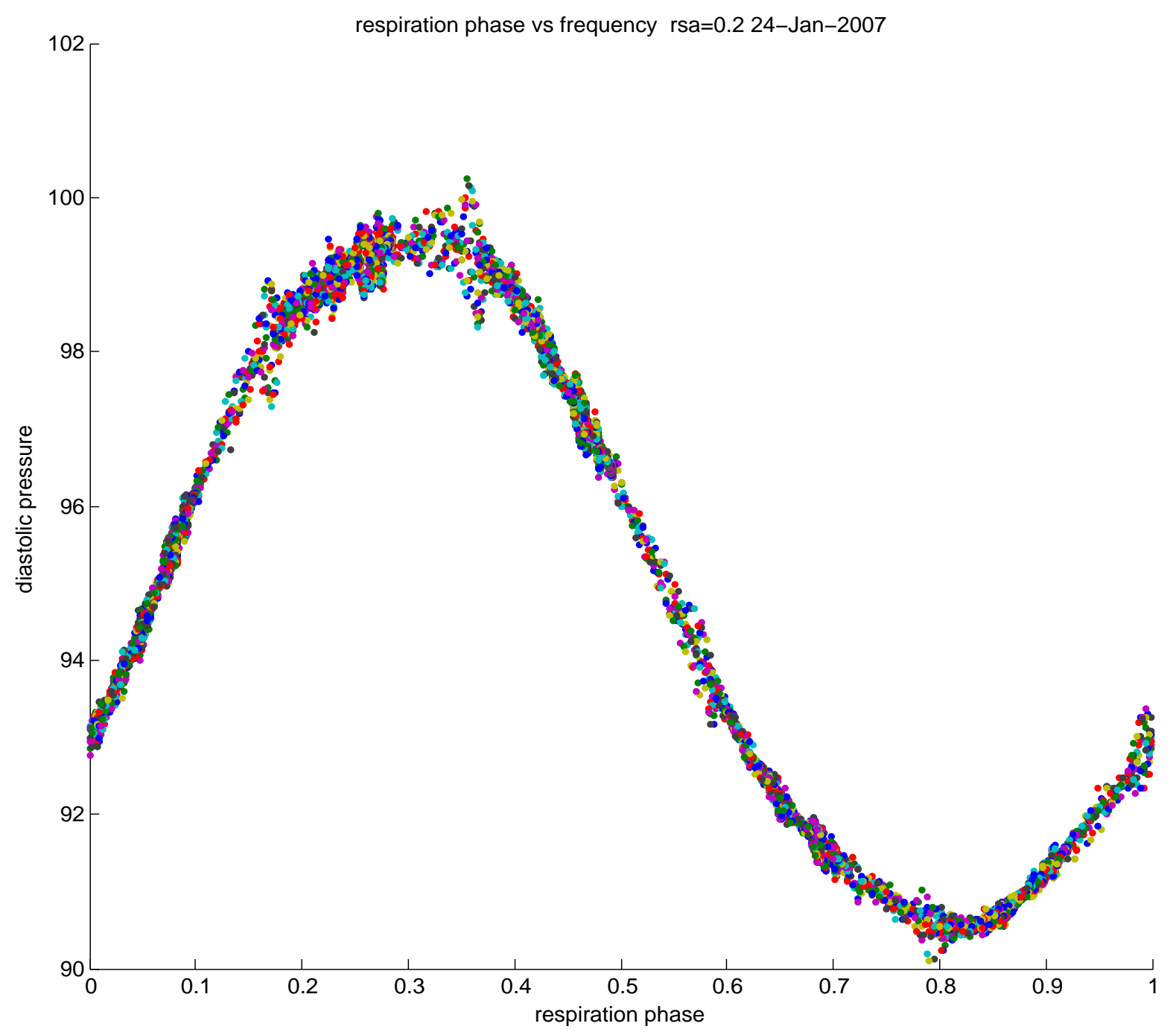

Figure 5.19: The change in diastolic pressure with respiration phase is independent of the locking zone and respiration frequency 


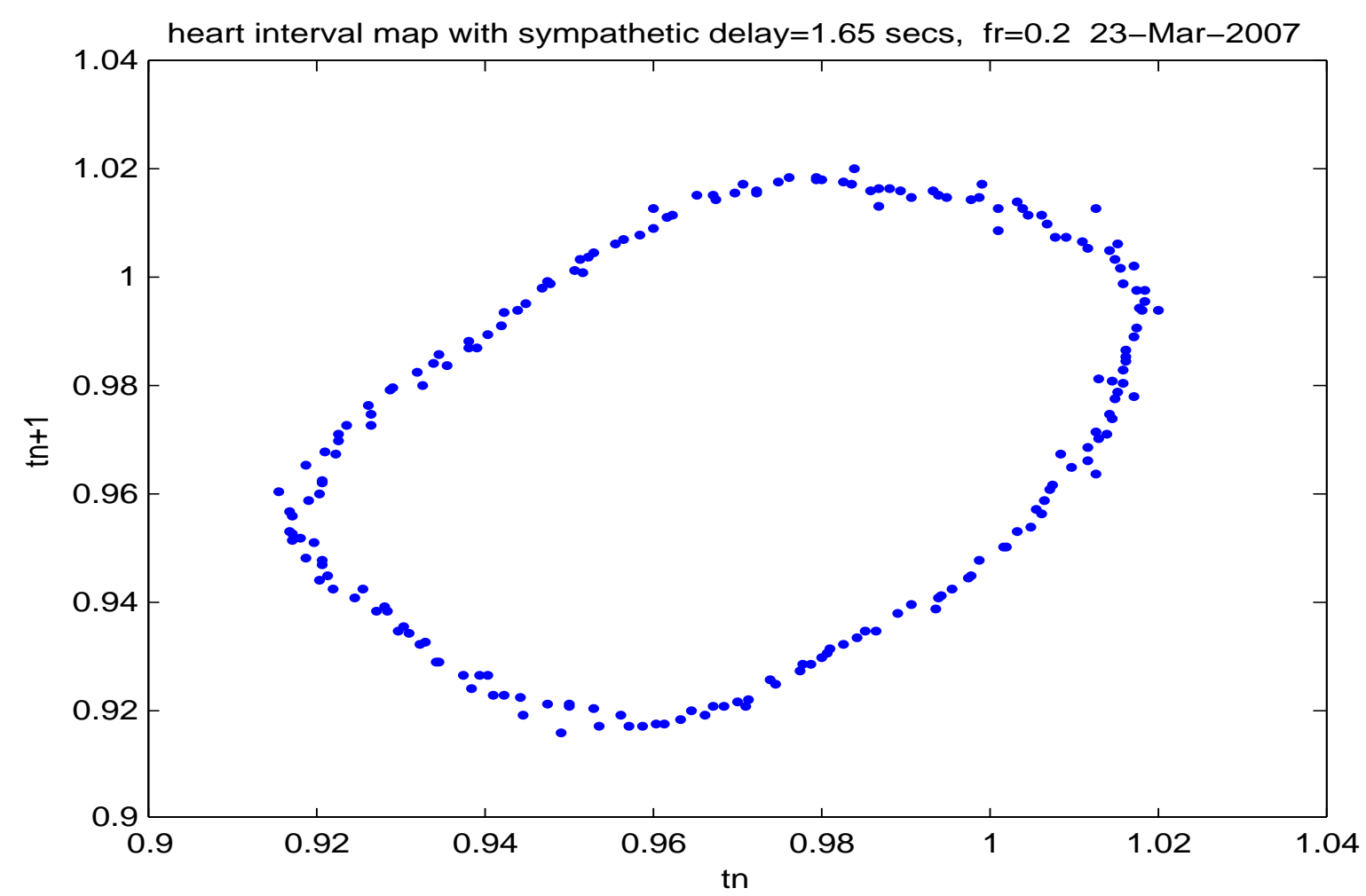

Figure 5.20: heart interval return map 
Figure 5.21 shows the dependence of the heart interval length on the respiration phase at the start of the interval.

Figure 5.22 shows the heart interval map in terms of respiration phase Therefore the effect of the respiration phase on the heart interval return time is shown graphically. 


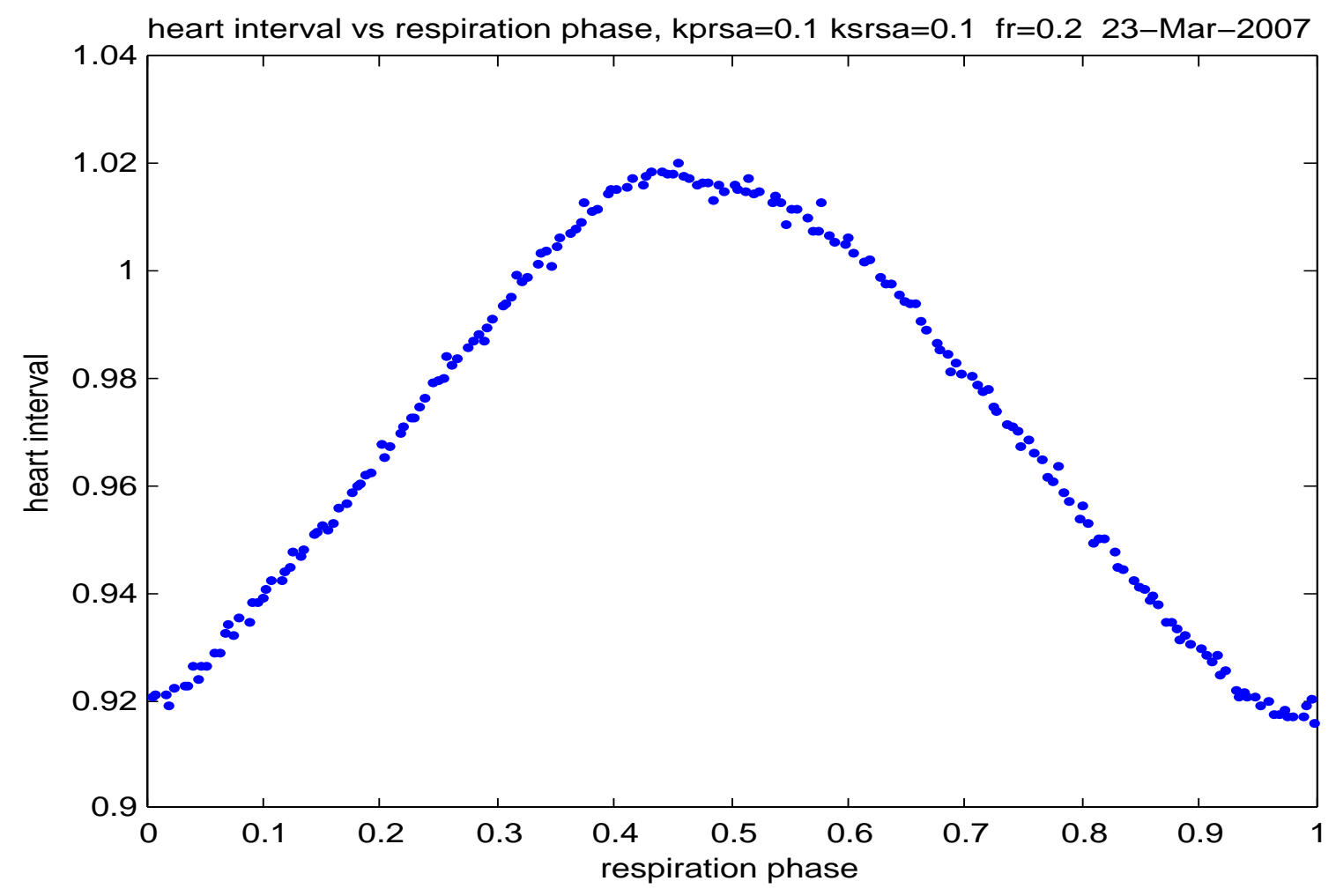

Figure 5.21: change of heart interval with respiration phase 


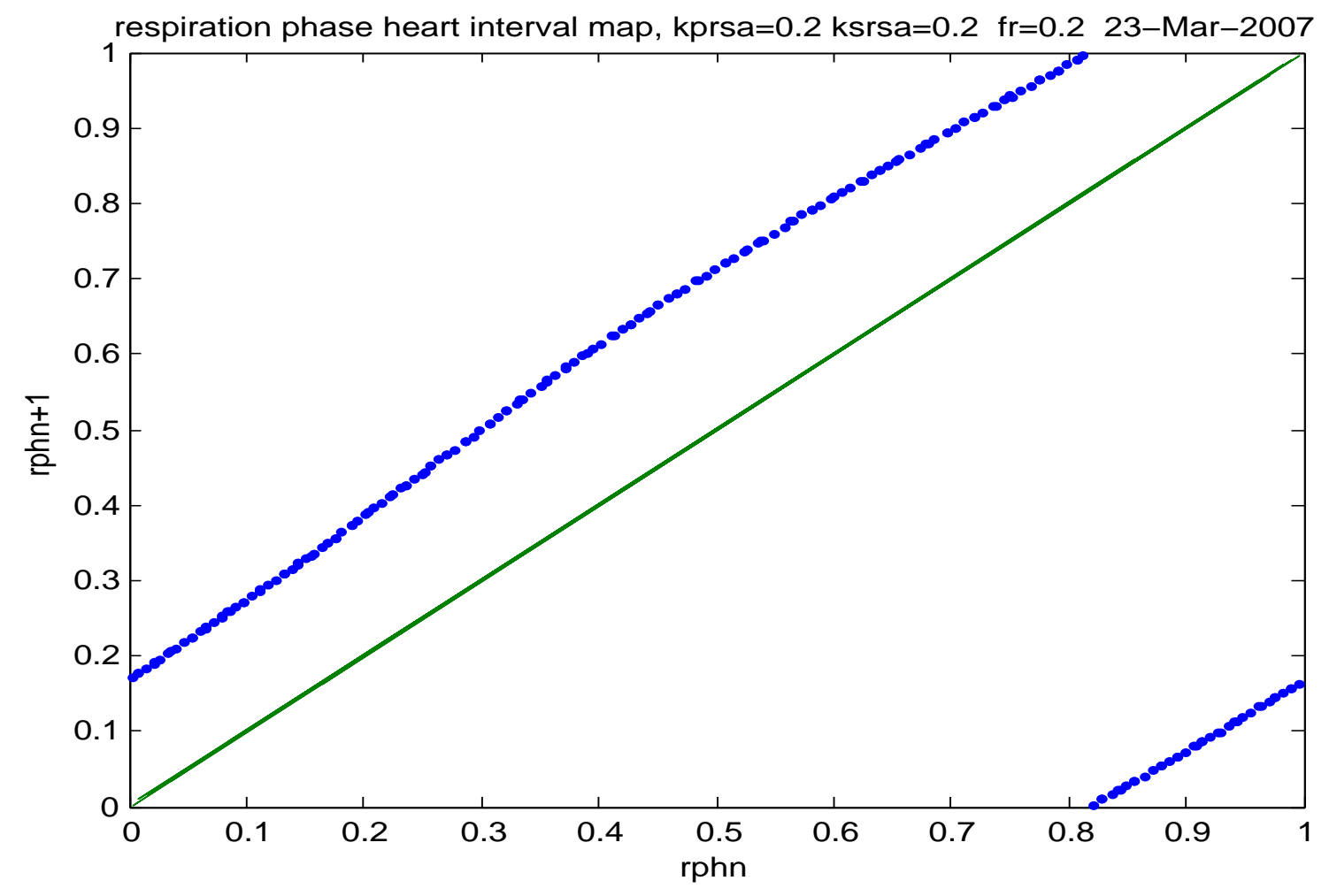

Figure 5.22: heart interval in terms of respiration phase, the RSA coupling is $2 \mathrm{x}$ nominal value 


\section{Chapter 6}

\section{Conclusions}

The purpose of the study was to investigate coupling in the human cardiovascular system. Mathematical models of oscillators were considered and the abstract mathematical representation of oscillating systems. Mathematical techniques for analysing oscillators were elaborated including how to understand the effects of noise, in particular white noise. Coupling between oscillators is divided into weak coupling amenable to perturbation methods or strong coupling requiring analytical solution or qualitative abstract models. The coupling can be assymmetric leading to the dynamics of the coupling being nonautonomous and disrupted by external dynamics. The dynamics of oscillator coupling is studied in terms of the phase difference between pairs of oscillators. Single order coupling dynamics are the most simple but any order dynamics are possible. In this case further state variables in addition to the phase difference lead to all possible dynamics for the phase difference.

The significant phenomenon of coupled oscillators is that of synchronisation where the frequency of two oscillators adjust to a ratio $\frac{N}{M}$ at a fixed phase difference, where $N$ and $M$ are integers. Another possible dynamical phenonemon produced by oscillator coupling is that of chaos. Otherwise the frequencies of the oscillators are incommensurate and the phase difference is said to be quasiperiodic. Synchronisation is defined 
to occur where $|N \theta-M \phi|=$ constant, where $\theta$ and $\phi$ are the phases of the oscillators, and $M, N$ are integers. In practice due to the nature of oscillator coupling as the oscillators progress through the $M$ and $N$ cycles respectively the phase difference $c$ is able to vary so that if it exceeds $\pm \pi$ then the oscillators have lost synchronisation. It is seen that this is a result of the restriction of the domain to the interval $[0,2 \pi]$, so that if the phase difference $\phi_{d}$ evolves outside the range $c \pm \pi$ then as the point $c-(\pi+\varepsilon)$ is identical to the point $c+(\pi-\varepsilon)$ an identical trajectory evolves in a different cycle, whereas if $\phi_{d}$ remains within $c \pm \pi$ the trajectory evolves in the one cycle of phase difference so that the oscillators remain synchronised.

Another useful quantity is the rotation number which is the ratio of the phase advance of the oscillators in the limit $\rho=\frac{1}{2 \pi} \lim _{N \rightarrow \infty} \frac{1}{N} \sum_{n=0}^{N}\left(\theta_{n+1}-\right.$ $\theta_{n}$ ) where the phase is measured in terms of cycles of the coupled oscillator.

Maps are used to study the dynamics of coupled oscillators. Forced oscillators have been extensively studied at least for weak coupling and they lead naturally to a 1D map as the driven oscillator phase is strobed by the fixed frequency of the forcing oscillator. The sine map $\phi_{n+1}=\phi_{n}+\tau+$ $b \sin \left(2 \pi \phi_{n}\right)$ is often used as a prototype map for the qualitative behaviour of such systems. The sine map is seen to exhibit locking zones. A 1D map is particulary useful applied to the phase model of two coupled 1D oscillators. Adding a dimension gives a 3D system where one oscillator has higher order dynamics or where the coupling is a two dimensional system. The geometry and mathematics of $2 \mathrm{D}$ maps as they pertain to coupled oscillators has been developed recently [23].

Where the behaviour of particular oscillating systems is needed then methods to analytically solve such systems are required. Solution often proceeds by perturbing the solution of a simplified system whose analytic solution is more easily obtainable. The method of averaging is seen to be a sound method for finding solutions of oscillating systems as it is based on the elimination of periodically varying quantities by averaging to obtain 
simplified equations for the important dynamics of a system. Similarly the method of angle/action variables was seen to be relevant to oscillating systems as it enables the reformulation of the oscillating system in terms of the oscillator angles and the interactions between the oscillators, and such a formulation is then amenable to solution by the method of averaging. Some properties and dynamics of Hamiltonian and non-conservative systems were remarked upon.

The Melnikov method and its relevance to understanding the effect of perturbations to homoclinic loops to saddle points on systems containing such dynamics was explained. That is the detection of chaotic regions of chaotic trajectories and of detecting synchronisation on periodic cycles.

There is still much work to do to arrive at an understanding of the dynamics of strongly coupled nonlinear oscillators. Perez and Glass [18] base their numerical study on the sine map and arrive at a bifurcation structure showing a wide range of complex dynamics the origin of which remains to be explained. Shilnikov et al. [23] goes further to the forced Van der Pol oscillator under the influence of a saddle point, a 3D system. While the range of complex dynamics is comparable to the sine map, there are structural differences in the bifurcations. Shilnikov et al. explain the dynamical basis of some of the bifurcations. Neither account explains the dynamics of the dependence of the locking range of the electronic phase locked loop on initial conditions. The locking region is determined by a saddle node bifurcation. A phase portrait given by Endo [40] shows where the detuning is above the critical value only one unstable manifold of the saddle point terminates on the stable fixed point and the other forms a homoclinic loop to the saddle point. So in this manner it differs from the driven Van der Pol oscillator where both unstable manifolds close on the stable fixed point. It is seen therefore that whereas Arnold tongues are generic for weak coupling between two oscillators, the bifurcation structure for strong coupling depends on the system. Some theoretical aspects remain to be explained and actual dynamical systems exhibited. 
In chapters 4 and 5 the human-cardiovascular system was modelled mathematically and the behaviour of the model described with particular attention to oscillator coupling in the system. Firstly some existing heart models were described, their principles and the cardiovascular system phenomena they are based on. A distinction is made between pulsatile models which model the beat to beat functioning of the cardiovascular system and non-pulsatile models which do not model the beat to beat fluctuations of the cardiovascular quantities but their average levels. As the current study concerns synchronisation of oscillators it is interested in the phase dynamics of oscillations in the cardiovascular system and uses a pulsatile model.

The model used is that of Seidel and Herzel [31] who studied bifurcations in the cardiovascular system but not synchronisation due to Respiratory Sinus Arrythmia (RSA). The study started with an implementation of the differential delay equation model of Seidel and Herzel in Matlab using the DDE23 differential delay equation solver. To verify the validity of the implementation some of the results of Seidel and Herzel were repeated. There was found to be strong qualitative agreement but some small variation quantitatively such as the exact bifurcation points. This did not seem to be significant. One interesting phenomenon reproduced was that of synchronisation between an alternans - arrhythmia of alternating heart interval length caused by increased parasympathetic loop gain and oscillations in the heart interval length caused by oscillations in fluid pressure which develop at low average fluid pressure. This was seen to involve the same mechanism as RSA synchronisation.

The RSA was studied across a range of respiration frequencies and a number of levels of coupling. Initially it was sought to map all ratios by sweeping the respiration frequency and searching at intervals of frequency for locking zones. This was not successful as the locking zones are quite small and difficult to locate for normal physiological parameters. Therefore the locking regions were found by estimating their location from 
the nominal respiration frequency and heart rate and plotting the rotation number across a small interval using small steps of respiration frequency so that the zones could be located by inspecting the plot. The range of the plot was then narrowed to uncover the detail of the locking zone.

It was observed that the width of the locking zones increased with coupling, and decreased with increasing denominator of the ratio. The mechanism of the frequency (and phase) locking was investigated. This was found to be due to the response of the parasympathetic loop being dependent on the phase of the heart interval. The influence of the respiration modulates the parasympathetic tone so that this expresses the phase of the respiration. The parasympathetic tone is multiplied by the phase effectiveness curve $F(\phi)$ which expresses the phase of the heart interval. This produces the phase difference term necessary for phase and frequency locking and also sum and difference terms at harmonics of the respiration and heart interval. The net effect of these terms averages out to zero over cycles of the $n$ cycles of heart interval and $m$ cycles of respiration of the period of synchronisation of a $\frac{n}{m}$ locking ratio. At most $m n$ oscillator cycles are required. From the Averaging Theorem the possible time scale for synchronisation is $\frac{1}{\varepsilon}$ where $\varepsilon$ is the coupling coefficient. But these higher order terms vary the relative phase of the heart interval over the respiration cycle and the heart interval time varies wrt the respiration phase.

Graphs of the heart interval in terms of respiration phase were plotted over a range of respiration frequencies to show that the heart intervals occured at fixed phases of the respiration cycle depending on the respiration frequency while the two oscillators were locked but the relative phases of the oscillators drifted when they were not locked. Fourier series of the phase effectiveness curve and the respiration influence were used to analyse these effects. Formally the phase locking of the heart system acts as a first order phase locked loop. From a phase plane plot the equilibrium points were seen to result from a tangent bifurcation and the width of the 
locking region can be determined from the loop gain.

The main result of the study is the smallness of the locking zones for normal physiological parameters. The RSA locking zones occupy only a small measure of the respiration frequency range as predicted by McGuinness et al. [26]. So it is difficult to locate minor ratios in large intervals of respiration frequency to exclude quasiperiodic behaviour. The heart system itself also affects the width of the Arnold tongues which can be explained in terms of the model. As the respiratory influence changes the fluid pressure it is counteracted by the action of the peripheral system controlling the peripheral resistance to restore the fluid pressure to its normal level and the peripheral resistance has the greatest effect on the fluid pressure. It was found that with fixed peripheral resistance the width of the locking zones was about $5 x$ greater. In this situation the respiratory influence is able to affect the sympathetic loops control of fluid pressure through heart interval length. Also the locking zones are dominated by the sympathetic loop gain as despite the counteracting effect of the parasympathetic loop they were $.5 x$ as wide with the sympathetic loop disabled. The effect of medical procedures on the cardiovascular system parameters could therefore well have a significant effect on RSA and this would need to be studied further in this case. The quantitative effect of cardiovascular coupling (CVC) on synchronisation needs to be modelled to determine its significance as this is another explanation for the physiological effect. 


\section{Bibliography}

[1] A. A. Brailove, "The dynamics of two pulse-coupled relaxation oscillators," Int. J. of Birfurcation and Chaos, vol. 2, no. 2, pp. 341-352, 1992.

[2] C. Peskin, Mathematical Aspects of Heart Physiology, pp. 268-278. New York: Courant Institute of Mathematical Sciences, New York University, 1975.

[3] R. Mirollo and S. Strogatz, "Synchronization of pulse-coupled biological oscillators," SIAM, J.Appl Math., vol. 50, pp. 1645-1662, 1990.

[4] Z. Wanzhen, L. Glass, and A. Shrier, "The topology of phase response curves induced by single and paired stimuli in spontaneously oscillating chick heart cell aggreagates," Journal of Biological Rhythms, vol. 7, no. 2, pp. 89-104, 1992.

[5] A. T. Winfree, The Geometry of Biological Time. New York, Heidelberg, Berlin.: Springer-Verlag, 1980.

[6] B. van der Pol, "The nonlinear theory of electric oscillators," Proceedings I.R.E., vol. 22, pp. 1051-1086, Sept 1934.

[7] R. FitzHugh, "Impulses and physiological states in theoretical models of nerve membrane," Biophys J, vol. 1, pp. 445-466, 1961.

[8] J. Nagumo, S. Arimoto, and S. Yoshizawa, "An active pulse transmission line simulating nerve axon," Proc IRE, vol. 50, pp. 2061-2070, 1962. 
[9] A. S. Pikovsky, M. G. Rosenblum, G. V. Osipov, and J. Kurths, "Phase synchronization of chaotic oscillators by external driving," Physica D, vol. 104, pp. 219-238, 1997.

[10] D. Gabor, "Theory of communication," Journal of the I.E.E., vol. 93, no. 3, pp. 429-457, 1946.

[11] M. G. Rosenblum, A. S. Pikovsky, and J. Kurths, "Phase synchronization of chaotic oscillators," Physical Review Letters, vol. 76, no. 11, pp. 1804-1807, 1996.

[12] J. D. Farmer, "Chaotic attractors of an infinite dimensional dynamical system," Physica D4, pp. 366-392, 1982.

[13] R. Stratnovich, Topics in the Theory of Random Noise, vols I,II. New York: Gordon and Breach, 1963.

[14] H. Z. Risken, The Fokker-Planck Equation. Berlin: Springer, 1989.

[15] Chandrasekhar, "Stochastic problems in physics and astronomy," Rev. Mod Phys, vol. 15, no. 1, 1943.

[16] G. M. Zaslavsky, "The simplest case of a strange attractor," Physics Letters, vol. 69A, no. 3, pp. 145-147, 1978.

[17] V. I. Arnold, "Small denominators i: Mappings of the circumference into itself," AMS Transl. series 2, no. 46, p. 213, 1965.

[18] L. Glass and R. Perez, "Fine structure of phase locking," Physical Review Letters, vol. 48, no. 26, pp. 1772-1775, 1982.

[19] V. I. Arnold, Geometrical Methods in the Theory of Ordinary Differential Equations. Springer-Verlag, 1983.

[20] P. Coullet, C. Tresser, and A. Arneodo, "Transition to turbulence for doubly periodic flows," Physics Letters, vol. 77A, no. 5, pp. 327-331, 1980. 
[21] A. S. Pikovsky, M. G. Rosenblum, and J. Kurths, "Phase synchronization in regular and chaotic systems: a tutorial," Int. J. of Bifurcation and Chaos, vol. 10, no. 10, pp. 2291-2306, 2000.

[22] P. Holmes and D. Rand, "Bifurcations of the forced van der pol oscillator," Quarterly of Applied Mathematics, pp. 495-509, January 1978.

[23] A. Shilnikov, L. Shilnikov, and D. Turaev, "On some mathematical topics in classical synchronization. a tutorial," International Journal of Bifurcation and Chaos, vol. 14, no. 7, pp. 2143-2160, 2004.

[24] S. Wiggins, Global Bifurcations and Chaos. Springer-Verlag, 1988.

[25] F. Verhulst, Nonlinear Differential Equations and Dynamical Systems. Springer-Verlag, 1990.

[26] M. McGuinness, Y. Hong, D. Galletly, and P. Larsen, "Arnold tongues in human cardiorespiratory systems," Chaos, vol. 14, no. 1, pp. 1-6, 2004.

[27] R. Deboer, J. Karemaker, and J. Strackee, "Hemodynamic flutuations and baroreflex sensitivity in humans: a beat-to-beat model," Am. J. Physiol.(Heart Circ. Physiol. 22), no. 253, pp. 680-689, 1987.

[28] J. T. Ottesen, "Modelling of the baroreflex-feedback mechanism," J. Math. Biol., vol. 36, pp. 41-63, 1997.

[29] A. C. Fowler and M. McGuinness, "A delay recruitment model of the cardiovascular control system," Journal of Mathematical Biology, vol. 51, no. 5, pp. 508-526, 2005.

[30] C.-C. Chen, "Threshold effects on synchronization of pulse-coupled oscillators," Physical Review E, vol. 49, no. 4, pp. 2668-2672, 1994.

[31] H. Seidel and H. Herzel, "Bifurcations in a nonlinear model of the baroreceptor-cardiac reflex," Physica D, no. 115, pp. 145-160, 1998. 
[32] E. Mosekilde and O. Mouritsen, eds., Modelling the Dynamics of Biological Systems, ch. x, pp. 205-229. Springer-Verlag, 1995.

[33] L. Shampine and S. Thompson, "Solving ddes in matlab," Applied Numerical Mathematics, vol. 37, pp. 441-458, 2001.

[34] M. Schwartz, Information, Transmission, Modulation, and Noise. McGraw-Hill, 1990.

[35] U. Niklasson, U. Wiklund, P. Bjerle, and B.-O. Olofsson, "Heartrate variation: what are we measuring?," Clinical Physiology, vol. 13, pp. 71-79, 1993.

[36] R. W. DeBoer, J. M. Karemaker, and J. Strackee, "Comparing spectra of a series of point events particularly for heart rate variability data," IEEE Transactions on Biomedical Engineering, vol. BME-31, pp. 384-387, April 1984.

[37] M. Brennan, M. Palaniswami, and P. Kamen, “Do existing measures of poincaré plot geometry reflect nonlinear features of heart rate variability?," IEEE Transactions on Biomedical Engineering, vol. 48, pp. 1342-1347, November 2001.

[38] J. Mateo and P. Laguna, "Improved heart rate variability signal analysis from the beat occurrence times according to the ipfm model," IEEE Transactions on Biomedical Engineering, vol. 47, pp. 985-996, August 2000.

[39] L. Keselbrener and S. Akselrod, "Selective discrete fourier transform algorithm for time-frequency analysis: Method and application on simulated and cardiovascular signals," IEEE Transactions on Biomedical Engineering, vol. 43, pp. 789-802, August 1996.

[40] T. Endo, "A review of chaos and nonlinear dynamics in phase-locked loops," Journal of the Franklin Institute, vol. 331, no. 6, pp. 859-902, 1994. 
[41] D. T. Kaplan and M. Talajic, "Dynamics of heart rate," Chaos, vol. 1, no. 3, pp. 251-256, 1991.

[42] A. C. Fowler and G. Kalamangalam, "Periodic breathing at high altitude," IMA Journal of Mathematics Applied in Medicine and Biology, no. 19, pp. 293-313, 2002. 


\section{Appendix A}

\section{Physiology of the Cardiovascular System}

The cardiovascular and respiratory systems are two examples of biological systems controlled by oscillators. These systems are coupled both centrally through nerve connections and mechanically through the heart and the lungs both being located in the chest cavity. The heart is controlled by the sino-atrial node, a small area of specialized muscle tissue on the outside of the heart at the top. The sino-atrial node autonomously generates the electrical impulses that trigger the periodic contractions of the heart muscle.

Respiration is centrally controlled by the medulla, a part of the brain stem. Three distinct groups of neurons in the medulla interact to operate the mechanism of breathing. The dorsal respiratory group controls inspiration. This centre spontaneously commences to generate the nervous activity which drives the diaphragm to contract and inflate the lungs. This is a steadily increasing rate of electrical impulses which are conducted by the phrenic nerve to the diaphragm. The depth of inspiration of the lungs is in direct response to the increasing activity of the dorsal group. Subsequently the pneumotaxic group quenches the activity of the dorsal group and expiration occurs through the elasticity of the lung tissue and muscle 
tissue. One nervous input which provokes the pneumotaxic group to act is from stretch receptors in the lungs which indicate the depth of the respiration to prevent tissue damage from overinflation. Other nervous inputs signal the level of $\mathrm{CO}_{2}$ and $\mathrm{O}_{2}$ in the blood. A third region of neurons the ventral group is usually dormant when the body is at rest but becomes active to increase breathing rate when $\mathrm{CO}_{2}$ and $\mathrm{O}_{2}$ ventilation requirements increase, acting both during the inspirational time to increase inspiration depth and decrease inspiration time and also during the expirational period, speeding expiration by driving muscle tissue to compress the chess cavity rather than relying on tissue elasticity.

The working of the heart pumps blood around the body through the arteries, capillaries, and veins, and thereby transports oxygen to the body and removes carbon dioxide from the body. For this there are two blood circuits, the pulmonary through the lungs which absorbs $\mathrm{O}_{2}$ from the air in the lungs and releases $\mathrm{CO}_{2}$ from the blood to the air in the lungs, and the systemic circuit which transports blood around the body to the other organs and muscles. The pressure of the blood in the arteries built up by the beating of the heart causes it to flow through the capillaries, at a rate determined by the dilation of the capillaries, to the veins which return the blood to the heart. One half of the heart receives venous blood from the body and pumps it through the pulmonary artery to the lungs to be reoxygenated. Oxygen rich blood from the lungs is carried by the pulmonary vein back to the heart to be pumped around the body. The contraction of the heart muscle forces the blood into the aorta, the main artery leading from the heart to the body, which expands to contain the blood. The elastic tension of the aorta maintains the pressure that is necessary for the blood to flow around the body. The pressure differential between the arteries and the veins maintains the flow through the capillaries. Immediately after a heart beat the pressure in the aorta is at a peak. A network of arteries transports the blood to different parts of the body where it flows through the capillaries and $\mathrm{O}_{2}$ and $\mathrm{CO}_{2}$ from body tissue are exchanged. A signif- 
icant division of the systemic circulation is between the brain and the rest of the body due to the importance of the brain and its large oxygen consumption. The rate of flow of blood through the capillaries is controlled by their dilation and contraction acting under nervous influence. The elasticity of the aorta and the resistance of the capillaries to blood flow lead to the Windkessel model for blood pressure in the arteries. The resistance of the capilleries to blood flow is denoted as $\mathrm{R}$. This is not a constant but varies according to the workings of the body. So $\Delta \mathrm{P}=\mathrm{F}$. $\mathrm{R}$, where $\Delta \mathrm{P}$ is the difference between the aortic pressure and the venous pressure and $\mathrm{F}$ is the blood flow in units of volume per second. This rule is analogous to Ohm's law in electronics. Therefore if the tension of the aorta can be compared to the voltage on a capacitor where the volume of blood is analogous to the electronic charge then $\frac{\mathrm{dP}}{\mathrm{d} t}=\frac{\mathrm{F}}{\mathrm{C}}$, and $\mathrm{C}$ is the ratio of units of volume of blood to units of tension of the aorta. Therefore $\frac{\mathrm{dP}}{\mathrm{d} t}=\frac{\Delta \mathrm{P}}{\mathrm{RC}}$ and the change in pressure in between heartbeats is proportional to the aortic pressure minus the venous pressure, with a time constant of RC. The pressure at the instant before the heart beat is known as the diastolic pressure and the peak pressure at the instant after the heart beat as the systolic pressure. The response of the artery wall to blood pressure is termed its compliance. This is the change in volume of an artery to a change in blood pressure. So $\mathrm{C}_{a}=\frac{\mathrm{dV}_{a}}{\mathrm{dP}_{a}}$. Therefore a heart stroke of volume $V_{v}$ changes the pressure in the artery by $\Delta \mathrm{P}=\frac{\mathrm{V}_{v}}{\mathrm{C}_{a}}$. The aorta and pulmonary arteries are physically different due to their different functions in the circulatory system. The aorta wall is thick and muscular so that it can exert a high pressure on the blood and force it around the systemic circuit. It is also long to conduct the blood to the organs of the body so this gives it a greater volume. The aorta compliance is therefore relatively low to maintain pressure on the blood it contains. The pulmonary artery is quite short, about $4 \mathrm{~cm}$ before it branches to each lung, as it only has to carry blood to the nearby lungs. It has thin walls and contains the volume of blood from the heart by expanding more. So it exerts a lower pressure on the blood than the aorta but it 
only has to force the blood through the lungs. Its compliance is about 19 times that of the aorta.

The heart beats autonomously under the control of the sino-atrial node. This node is a type of muscle tissue that is adapted to contract spontaneously and generates the electrical impulse that is conducted throughout the heart and initiates a heart muscle contraction. The bio-chemical process occurs through the flow of ions through the cell membrane. Three types of ions are involved, calcium, sodium, and potassium. $\left(\mathrm{Ca}^{++}, \mathrm{Na}^{+}\right.$ and $\mathrm{K}^{+}$). A momentary high $\mathrm{Ca}^{++}$inflow causes the electrical impulse, an outflow of $\mathrm{K}^{+}$causes the -ve polarisation of the cell membrane, and a steady inflow of $\mathrm{Na}^{+}$causes the subsequent depolarisation. Channels in the cell membrane open and close to transport the ions into or out of the cell. The voltage across the cell membrane is polarized to about $-60 \mathrm{mV}$ with respect to the outside of the cell and then slowly starts to increase. At a threshold level there is a discharge which sharply depolarizes the muscle cell causing the voltage pulse that triggers the heart beat.

The two separate pumps of the heart, one for the pulmonary circuit and one for the systemic circuit, act in the same way. They each have two chambers, the atrium and the ventricle. The atrium fills with venous blood during the time between beats when the heart is at rest. Thus the venous pressure does not increase, as the blood flows from the capillaries as the atrium inflates to contain the extra volume. It then operates to pump the blood through a valve into the ventricle which immediately operates to pump the blood into the pulmonary artery or aorta through another valve. It can be seen that the volumes of blood pumped by each side of the heart must be the same otherwise pressure would build up in either of the aorta or pulmonary artery due to increasing volume. The contractility of the heart refers to its tendency to contract more strongly according to the initial extension of muscle fibres, known as myocardial fibres, in the ventricle. A stronger contraction then equates to a higher artery pressure at the end of the contraction. So a longer interval between beats fills the atrium 


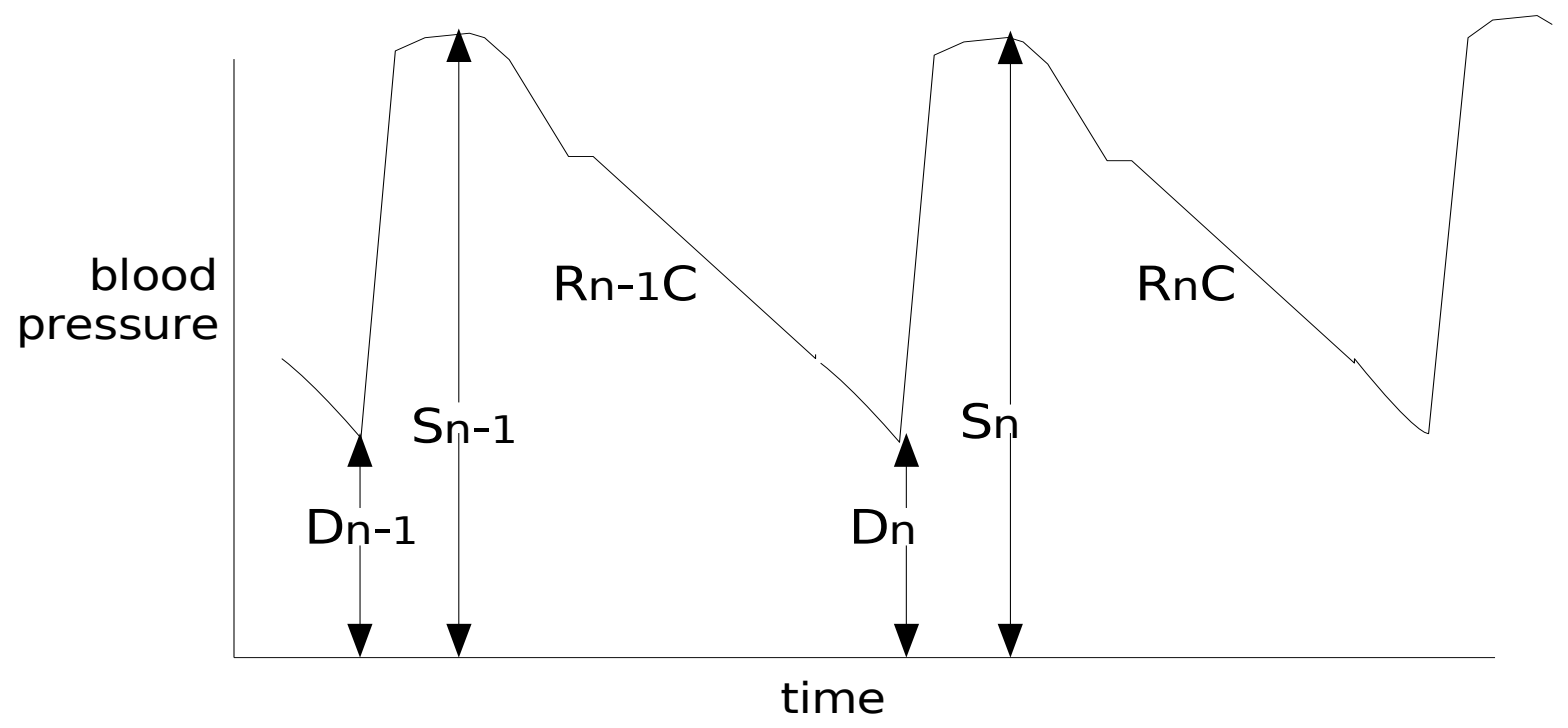

Figure A.1: Windkessel model of blood pressure, Dn diastolic pressure, Sn systolic pressure 


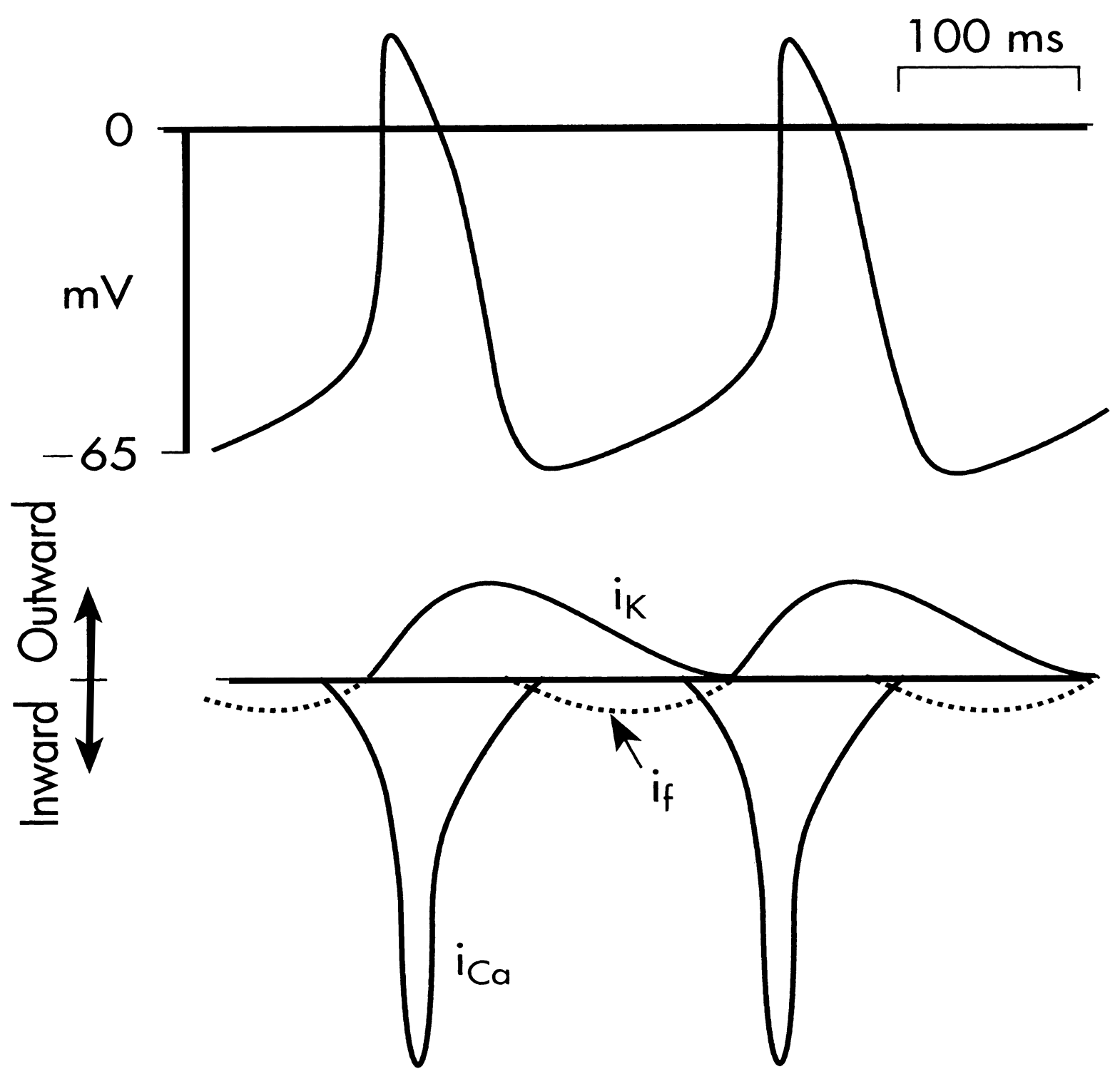

Figure A.2: ion flows of the sino-atrial node, $\mathrm{i}_{\mathrm{Ca}}=\mathrm{Ca}^{++}, \mathrm{i}_{\mathrm{K}}=\mathrm{K}^{+}, \mathrm{i}_{\mathrm{f}}=$ $\mathrm{Na}^{+}$, with corresponding cell membrane potential 
more completely and the increased blood volume extends the myocardial fibres which results in a stronger contraction. A mechanism known as the Frank-Starling mechanism maintains the equality of pressure between the systemic and pulmonary systems independent of heart rate, equalising the blood flows in each circuit. An amount of blood is present in the ventricle at the end of a contraction. Whatever the blood flow from the atrium due to the venous pressure and the length of the heart interval, if the strength of the contraction expels more blood from the ventricle than has been pumped into the ventricle then the residual blood in the ventricle falls and subsequently the ventricle is filled to a lower level so that the strength of the contraction falls to adjust to the inflow from the atrium or vice-versa. If the artery pressure builds up then the residual blood in the ventricle also increases and the contraction strength increases until the volume of blood from the atrium is expelled into the aorta and similarly if the artery pressure falls. So an increase in output at one ventricle will flow through to increased venous inflow at the otherside of the heart and the second ventricle will rise to match the first.

Although the heart can beat autonomously controlled by the sino-atrial node at a rate of about $60-70$ beats per minute a central control system modifies this rate in accordance with varying demands of the body. Thus blood pressure and $\mathrm{CO}_{2}$ and $\mathrm{O}_{2}$ levels alter the heart rate. A centre of neurons in the medulla (part of the brain stem) controls heart rate through the parasympathetic and sympathetic nervous systems. Afferent nerve signals from a variety and number of receptors in the vascular system travel to the medulla and the medulla transmits efferent nerve signals to the heart to change its rate. The parasympathetic signals are conducted by the vagal nerve from the medulla to the sino-atrial node. In the nervous system the parasympathetic nervous system accentuates the sympathetic nervous system in some functions. The paraysmpathetic and sympathetic nervous systems are distingushed by the type of neuro-transmitter that is released in response to nerve signals at the nerve endings where they con- 
tact the organ affected. The parasympathetic system uses acetylcholine as its neurotransmitter and the sympathetic system uses epinehrine also known as adrenaline. These neurotransmitters have distinct effects on the flow of ions through the cell membrane of muscle tissue. The effect of parasympathetic activity is to slow the heart down and so the acetylcholine mainly increases the inflow of $\mathrm{K}^{+}$resulting in a higher magnitude polarization which takes longer to discharge and so the heart rate is slowed. The flows of $\mathrm{Ca}^{++}$and $\mathrm{Na}^{+}$are also decreased slightly. The effect of sympathetic activity is to increase the heart rate as the epinehrine increases the flow of all ions but $\mathrm{K}^{+}$less so resulting in a quicker depolarization and faster heart rate. A significant difference between the two neurotransmitters is their response time. Acetylcholine is released in a delay of about .3 seconds so has an immediate effect on the heart rate. It is also metabolised straight away so does not build up in the tissue of the sinoatrial node. Epinehrine is released only slowly and in smaller amounts, so has to be released over a period of time to have any effect and then it is recovered by the body even more slowly so its action is longer lasting. The delay for the release of epinehrine is about 3 seconds. The effect of the epinehrine dynamics is like a low pass filter on the sympathetic nervous activity. In experimentation different anesthetics can be used to selectively block the parasympathetic or sympathetic actions to observe the effect on the cardiovascular system. Atropine can be used to block acetylcholine and Propranolol to block epinehrine. It is seen that the heart beat interval time varies according to the effects of control systems in the body with different response times. This is known as heart rate variability. The effect of these different systems is to give the heart rate variability a characteristic $\frac{1}{f}$ noise power spectrum. Alternatively the variability could be a centralised effect of the autonomic nervous system. The effect of the parasympathetic and sympathetic control loops can be seen to cause peaks in the power spectrum. Energy in the $.04 \mathrm{~Hz}-.15 \mathrm{~Hz}$ band is associated with the sympathetic control loop and energy in the $.15 \mathrm{~Hz}-.5 \mathrm{~Hz}$ band with parasymp- 
thetic conrol. A sharp peak at $.2 \mathrm{~Hz}$ is associated with respiration. Lower frequencies are the result of other systems of the body such as hormonal and endocrine systems. The power spectrum can be used diagnostically and these quantities designated as If energy and, hf energy indicate the activity or tone of the autonomic system and their ratio is also significant. A lower heart rate variability or increased sympathetic activity is associated with the occurence of pathological cardiac arrhythmia.([41])

To control blood pressure special baroreceptor cells in the arteries sense the blood pressure and output signals to the nervous system. Baroreceptor cells are located in the aorta and also in the branching of the carotid artery in the neck leading to the brain. This shows the importance of blood flow to the brain. Ordinarily there would not be much difference in the blood pressure at the two locations as they are directly connected but should the blood pressure in the brain drop, more blood is directed there through the constriction of capillaries in the body blocking blood flow through the body. The response of the baroreceptors has a characteristic S-shaped curve. so the nervous output is saturated at high and low pressure. The level of the pressure is shown by the rate of the electrical impulses that are conducted along the neurons of the nerve fibre, so for the parasympathetic nerve, at high pressure the spike rate is at a maximum and drops to a minimum at low pressure. The sympathetic system reponse to the baroreceptor is an inverted S-shape so that the spike rate is at a minimum at high blood pressure. In this way both the parasympathetic and sympathetic nervous systems respond to high blood pressure by decreasing the heart rate.

Blood pressure is mainly effected by the dilation and contraction of the capillaries. But it is only the sympathetic system which affects the capillaries. The parasympathetic system is not involved here. So the heart rate only has an effect on the blood pressure on a beat by beat basis due to the delay in the sympathetic control loop and the higher gain of the sympathetic loop. As mentioned blood flow in different parts of the body 


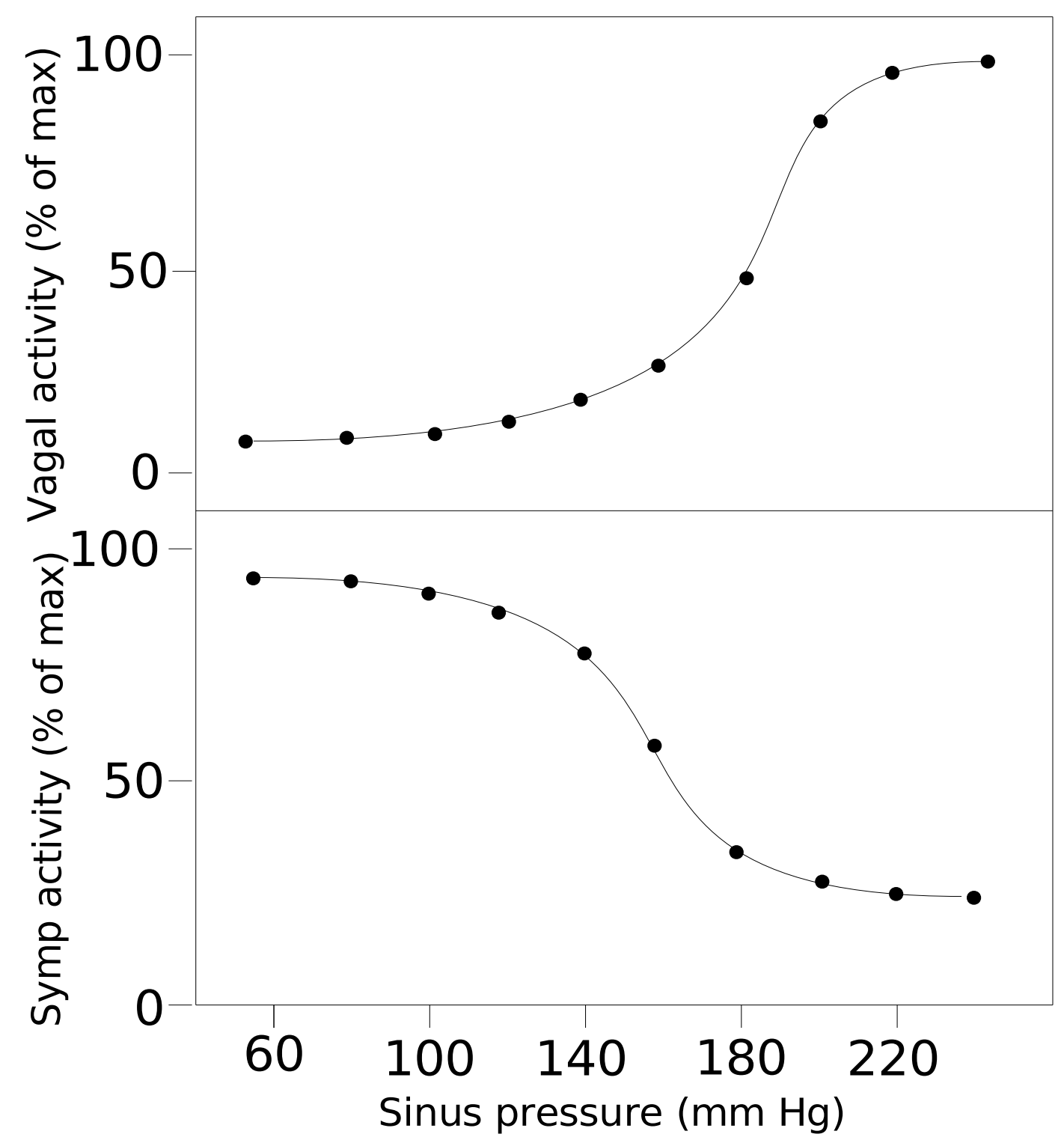

Figure A.3: baroreceptor S curves 
can be controlled separately and the capillaries are dilated through sympathetic control to increase blood flow and decrease blood pressure and vice versa. The 3 second delay in sympathetic control is illustrated by the phenomonen of Mayer waves. Mayer waves are oscillatory fluctuations in blood pressure with a period of about 10 seconds. The 10 second period is a consequent of the 3 second delay in the sympathetic control loop and oscillation is caused by the gain of the loop. [29]

The levels of $\mathrm{CO}_{2}$ and $\mathrm{O}_{2}$ in the blood are controlled both through the ventilation rate and through the rate of blood flow. Chemoreceptor cells detect the levels of $\mathrm{CO}_{2}$ and $\mathrm{O}_{2}$ in the blood and signal this to the neuron centres in the medulla controlling respiration and heart rate. These chemoreceptor cells are located in the aorta and also the branching of the carotid artery in the neck. $\mathrm{CO}_{2}$ and $\mathrm{O}_{2}$ levels are detected by different chemoreceptors. Additionally the dorsal nerve group in the medulla responsible for inspiration responds directly to $\mathrm{CO}_{2}$ in the blood. Its metabolic rate increases in direct response to an increase in $\mathrm{CO}_{2}$ in the blood. Thus the ramp of nervous activity driving the diagphram to contract will increase at a faster rate as the $\mathrm{CO}_{2}$ level increases. The $\mathrm{CO}_{2}$ and $\mathrm{O}_{2}$ chemoreceptors are not independent. They affect each others response. This effect is known as the Oxford fan.

At normal $\mathrm{CO}_{2}$ and $\mathrm{O}_{2}$ levels it is the $\mathrm{CO}_{2}$ which control ventilation and the $\mathrm{O}_{2}$ receptors are relatively ineffective, except at extremes of oxygen pressure. But as the $\mathrm{CO}_{2}$ pressure departs further from its normal level then the $\mathrm{O}_{2}$ receptors have more effect. A recognised abnormality in breathing is called Cheyne-Stokes breathing after the physicians who discovered it. In this condition breathing is periodic rising to a peak of inspiration depth and then falling possibly to stop momentarily. Two situations where this occurs are in heart disease and at high altitudes in unacclimatised persons. The two cases can be distingushed by the length of the period of breathing. For heart disease a weak heart results in a slow blood flow and the delay in the blood reaching the peripheral chemore- 


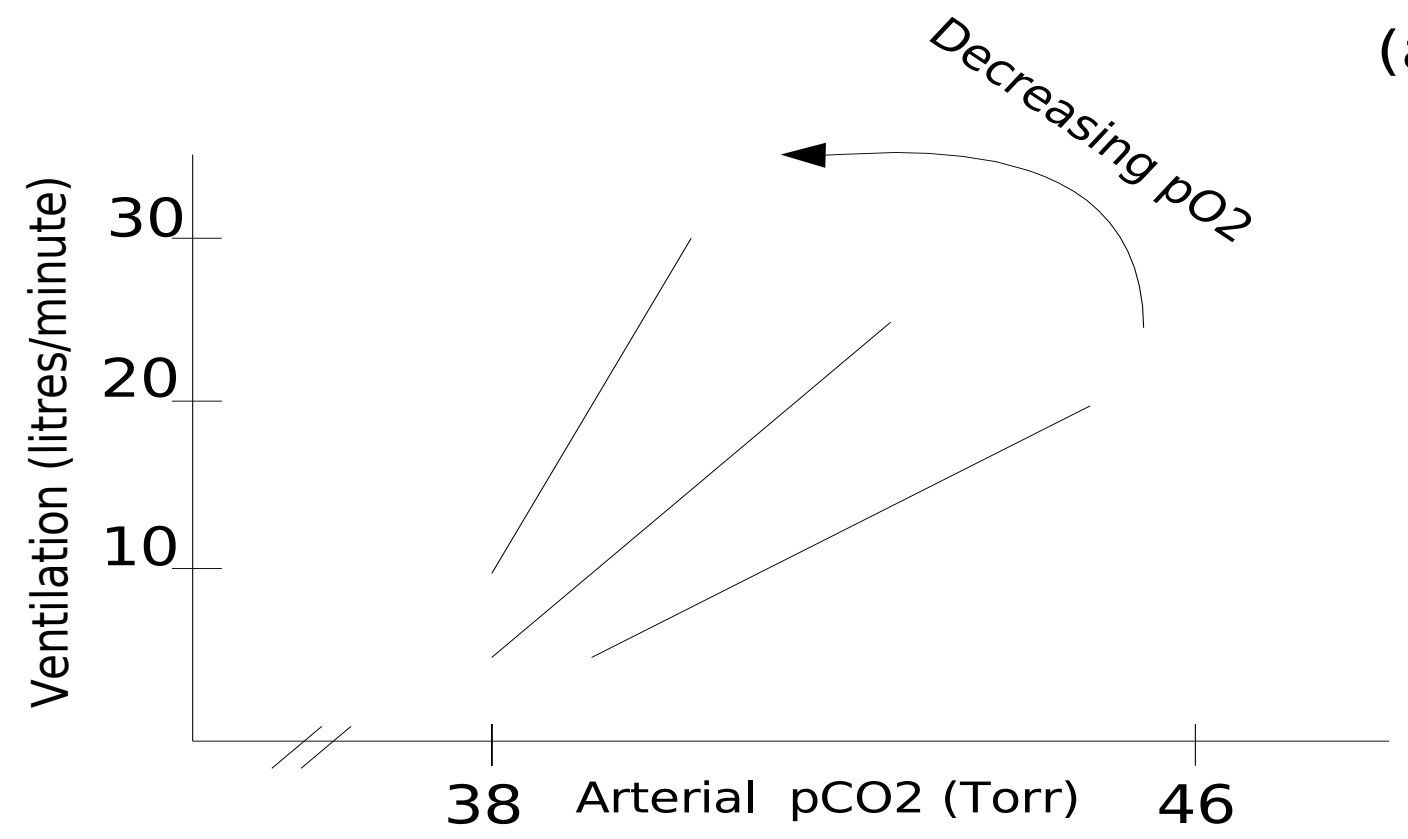

(a)

(b)

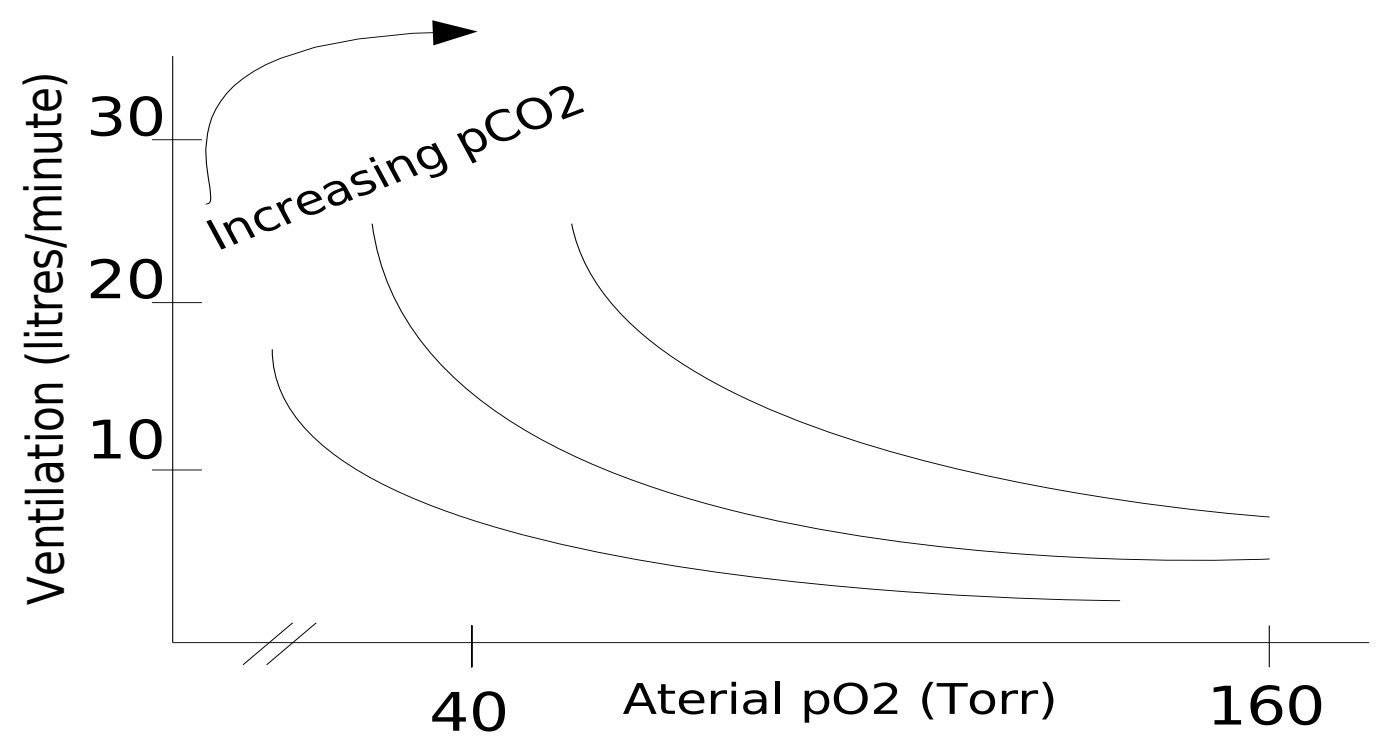

Figure A.4: Oxford fan 
ceptors in the carotid artery causes an oscillation in breathing of about 60 seconds period. In the case of unacclimatised persons at high altitudes it is the change in gain of the peripheral chemoreceptors due to the Oxford fan effect which results in a breathing depth oscillation of about 20 seconds period. Physiologically a reduction in $\mathrm{O}_{2}$ pressure in the blood due to the decrease of $\mathrm{O}_{2}$ pressure in the air at altitude, increases blood flow and that along with the altitude also reducing blood $\mathrm{CO}_{2}$ pressure renders the central controller ineffective. The lower $\mathrm{CO}_{2}$ pressure in the blood accentuates the response of the peripheral controller, which is now controlling ventilation, to $\mathrm{O}_{2}$, resulting in breathing depth fluctuation and fluctuating $\mathrm{O}_{2}$ levels. ([42])

There is direct coupling between the two systems of heart rate and respiration as a result of the physiology. Respiratory sinus arrhythmia (RSA) is coupling from respiration to heart rate. Effectively heart rate increases during inspiration and decreases during expiration. There are two possible sources, central coupling in the medulla, the more significant, and mechanical coupling in the chest cavity. The central coupling acts by diminishing parasympathetic activity during inspiration so that the heart rate is allowed to increase to its sinoatrial determined rate or the rate due to sympathetic activity.

Mechanical coupling occurs due to the increase and decrease in air pressure in the chest cavity during respiration. During inspiration the lower air pressure necessary to inflate the lungs also increases the filling of the atrium of the heart by lower pressure venous blood leading to a greater stroke volume of the heart and an increase in blood pressure in the aorta. Apparently this would lead to a decrease in heart rate due to an increase in parasympathetic activity. However an additional physiological mechanism is at work in the form of stretch receptors in the atrium which affect the sinoatrial node as the atrium is inflated and in the absence of other nervous input, speed the heart up. The activity of the stretch receptors in the atrium counteracts parasympathetic activity affecting the sinoatrial 
node which is in any case diminished by central coupling. For low initial volumes of blood the heart rate does in fact decrease as paraysmpathetic activity dominates, but as volume increases further heart rate speeds up again when the stretch receptors in the atrium take effect.

Coupling from the heart to the respiration is known as cardiovascular coupling (CVC). This has the effect of causing a new cycle of respiration to be synchronised to a heartbeat. That is at the end of expiration the next inspiration is triggered by a heartbeat. The cause of this is not clear. Perhaps a new charge of blood from the heart affects the central chemoreceptor. Clearly an integral number of heart beats occurs in each respiration cycle when there is synchronism. But also the synchronisation might skip a cycle or cycles so the ratio of heart beats to respiration becomes $m: n$. 


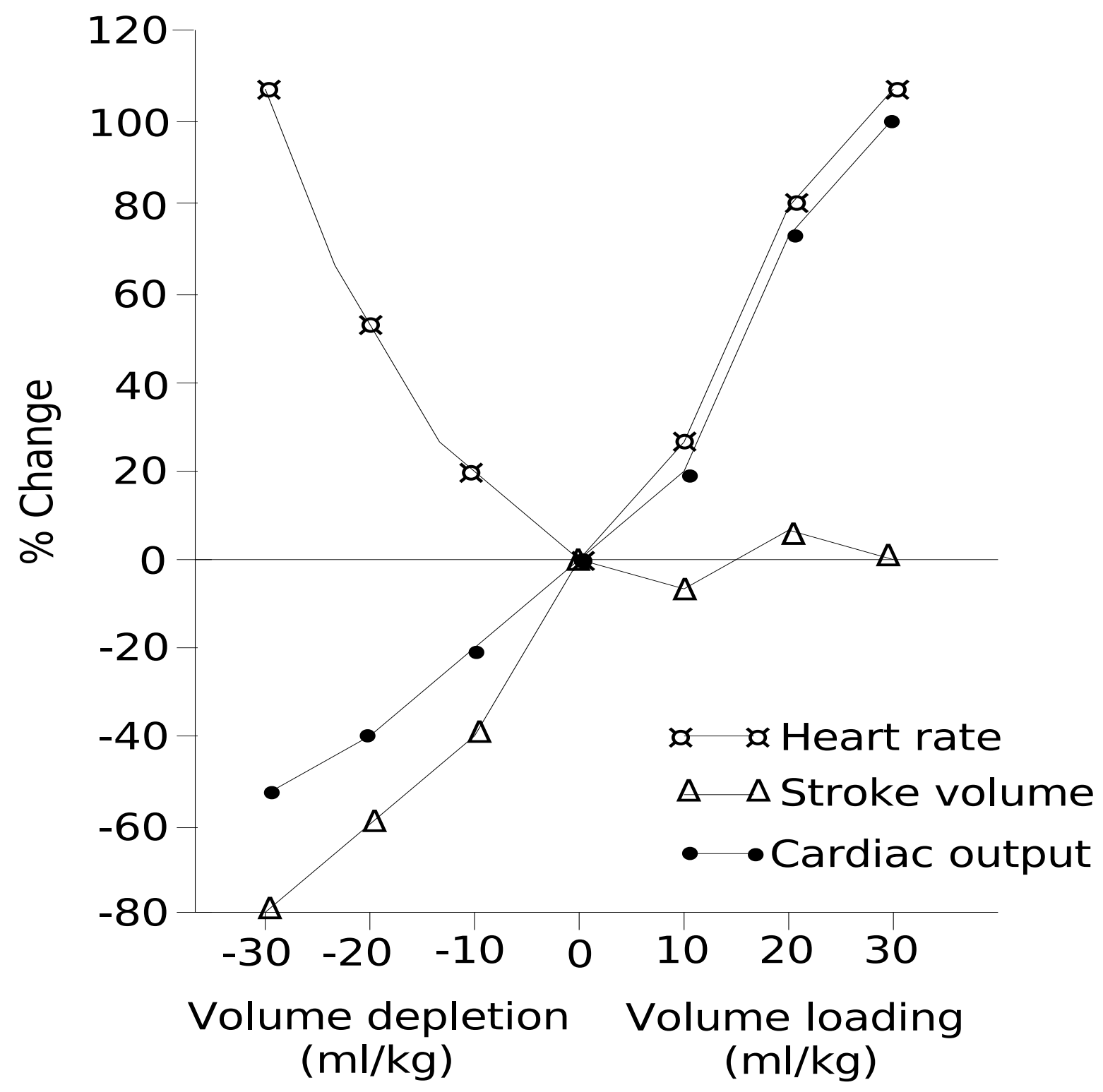

Figure A.5: heart rate vs blood volume 